\title{
Multibond Forming Tandem Reactions of Anilines via Stable Aryl Diazonium Salts: One-Pot Synthesis of 3,4-Dihydroquinolin-2-ones
}

\author{
Réka J. Faggyas, Megan Grace, Lewis Williams, and Andrew Sutherland*(i) \\ WestCHEM, School of Chemistry, The Joseph Black Building, University of Glasgow, Glasgow G12 8QQ United Kingdom
}

Supporting Information

ABSTRACT: A fast and effective one-pot tandem process that generates Heck coupled products from readily available anilines via stable aryl diazonium tosylate salts was developed. The mild and simple procedure involves rapid formation of aryl diazonium salts using a polymer-supported nitrite reagent and $p$-tosic acid, followed by a base-free Heck-Matsuda coupling with acrylates and styrenes. Using 2-nitroanilines as substrates, the one-pot tandem process was extended for the direct synthesis of 3,4-dihydroquinolin-2-ones. In this case, following diazotization and Heck-Matsuda coupling to give methyl cinnamates, addition of hydrogen and reutilization of the palladium catalyst for reduction of the nitro group and hydrogenation of the alkene resulted in efficient formation of

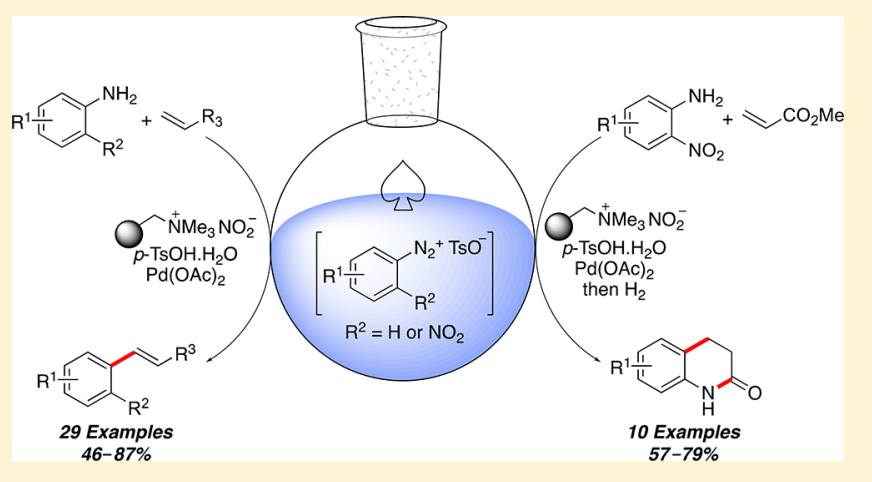
3,4-dihydroquinolin-2-ones. The synthetic utility of this one-pot, four-stage process was demonstrated with the five-pot synthesis of a quinolinone-based sodium ion channel modulator.

\section{INTRODUCTION}

The Mizoroki-Heck coupling of aryl halides with olefins in the presence of a palladium catalyst and a base has become a general method for carbon-carbon bond formation. ${ }^{1}$ This powerful transformation allows the stereoselective synthesis of highly substituted alkenes, key synthetic building blocks for organic chemistry. ${ }^{2}$ Despite the many applications of the Mizoroki-Heck reaction, the transformation can be limited by the requirement of a base, elevated temperatures, and olefin migration. A less-utilized variant of this cross-coupling is the Heck-Matsuda reaction that uses aryl diazonium salts as more reactive aryl halide surrogates (Scheme 1a). ${ }^{3}$ The increased electrophilic nature of aryl diazonium salts allows the HeckMatsuda reaction to proceed at lower temperatures and often without the need for ligands or a base. ${ }^{4}$ Under these milder conditions, alkene migration is minimized, generating cleaner products.

Despite these advantages, the safety hazards associated with aryl diazonium salts has restricted the widespread use of the Heck-Matsuda reaction. This has partly been countered by replacing aryl diazonium chlorides and acetates that are unstable above $0{ }^{\circ} \mathrm{C}$, with more stable tetrafluoroborate crystalline salts. ${ }^{5}$ However, these compounds still require storage at low temperatures and in the dark. To minimize the safety issues of handling and storing aryl diazonium salts, onepot methods in which the aryl diazonium salts are formed in situ and directly subjected to a Heck-Matsuda reaction have been developed. ${ }^{6}$ Examples include a procedure reported by Andrus and coworkers that used an imidazolium carbene as a palladium ligand for the base-free coupling of a focused series of substrates in $17-62 \%$ yield (Scheme $1 \mathrm{~b}) .^{7}$ In recent years, the Felpin group made a major contribution in understanding and advancing the applications of the Heck-Matsuda reaction. ${ }^{8}$ This included the development of a one-pot tandem synthesis of Heck adducts from anilines using catalytic amounts of diazonium salts via a double catalytic cycle (Scheme 1c). ${ }^{8 c}$ This process was found to be general for the efficient coupling of a wide range of substituted anilines with methyl acrylate, giving the Heck adducts in excellent yields over a reaction time of $48-65 \mathrm{~h}$.

In 2008, the groups of Filimonov and Chi reported the synthesis and characterization of aryl diazonium tosylate salts. ${ }^{9}$ These were prepared from anilines using a polymer-supported nitrite reagent under mild conditions and were found to have particularly high thermal and aging stability. Despite these properties, aryl diazonium tosylate salts are still reactive and have been utilized in standard substitution and cross-coupling reactions. ${ }^{9,10}$ We recently demonstrated that aryl diazonium tosylate salts could be generated in situ and subjected to an iodination reaction for the one-pot tandem synthesis of aryl iodides from anilines. ${ }^{11}$ Because of the relatively stable nature of aryl diazonium tosylate salts and this initial demonstration of their application in one-pot tandem processes, we were interested in further exploiting these compounds for additional multistep transformations. Herein, we now report a rapid, mild, and nonhazardous one-pot tandem diazotization and Heck reaction of anilines for the general preparation of cinnamates

Received: July 26, 2018

Published: September 19, 2018 
Scheme 1. Synthesis of Heck Adducts from Anilines

a) Standard Heck-Matsuda reaction process from anilines<smiles>[R]C=Cc1ccc[R1]([R])c1</smiles>

b) One-pot diazotization and Heck-Matsuda reaction

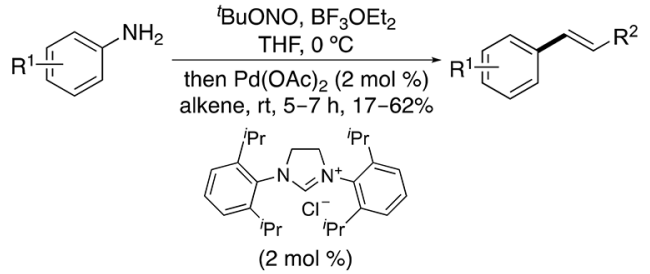

c) One-pot synthesis of methyl cinnamates from anilines using catalytic amounts of diazonium salts

$$
\begin{aligned}
& \underset{\begin{array}{c}
\mathrm{Pd}(\mathrm{OAc})_{2}(2.2 \mathrm{~mol} \%) \\
\text { methyl acrylate }
\end{array}}{\mathrm{MeSO}_{3} \mathrm{H}(20 \mathrm{~mol} \%)} \\
& \text { rt, } 48-65 \text { h, 51-99\% }
\end{aligned}
$$

d) This work: One-pot processes using stable aryl diazonium tosylate salts

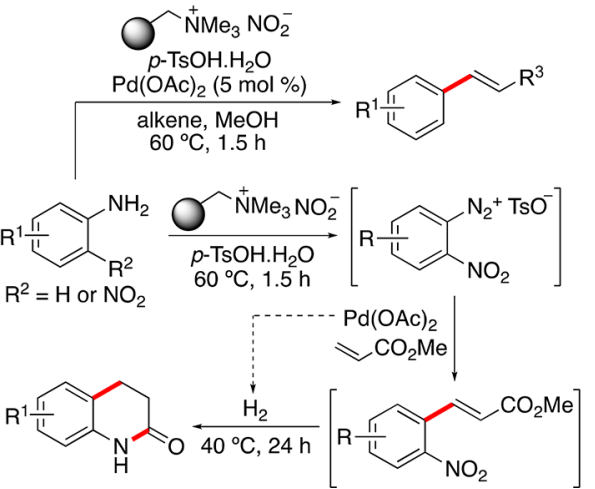

and styrenes (Scheme 1d). The highly chemoselective nature of this tandem process is demonstrated with halogenated anilines that following diazotization and Heck reaction can undergo further functionalization via additional cross-coupling reactions. We also describe an extension of the tandem process with 2-nitroanilines in which the Heck adducts can be directly converted to 3,4-dihydroquinolin-2-ones by the addition of hydrogen and reutilization of the palladium catalyst.

\section{RESULTS AND DISCUSSION}

In several studies of the Heck-Matsuda reaction, nitrosubstituted anilines have been found to be problem substrates. $^{5,6 \mathrm{~d}, 12}$ The high redox potential and a favorable homolytic dediazonization pathway of nitro-substituted aryl diazonium salts have been used to explain the facile decomposition of these compounds and the complex mixtures from attempted Heck-Matsuda reactions. Felpin and coworkers demonstrated that their one-pot diazotization and Heck-Matsuda reaction process could overcome these issues, allowing the efficient generation of coupled products (Scheme 1c). ${ }^{8 c}$ Because of the issues associated with nitro analogues and to investigate whether our one-pot tandem process could also overcome these problems, our study began by investigating the one-pot synthesis of aryl diazonium tosylate salts and HeckMatsuda coupling reaction using 4-nitroaniline (1a) as a substrate. Initially, a one-pot tandem process in which all the reagents were added together was investigated. This included the polymer-supported nitrite reagent, which prevents the release of nitrogen oxides and is easily prepared by the ion exchange of tetraalkylammonium functionalized resins, such as Amberlyst A-26 with aqueous solutions of sodium nitrite. ${ }^{9,11}$ Initially, the use of tetrafluoroboric acid as a proton source was compared with $p$-tosic acid (Table 1, entries 1 and 2). A higher

Table 1. Optimization of the One-Pot Tandem Diazotization and Heck-Matsuda Reaction

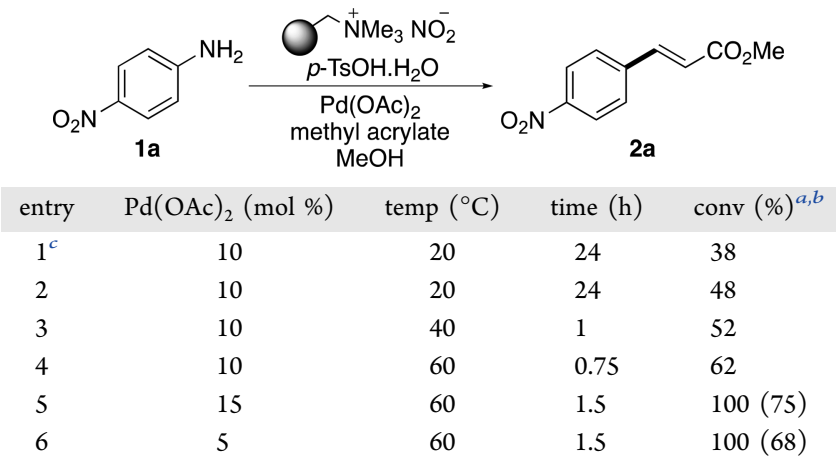

${ }^{a}$ Conversions were measured using 1,3,5-trimethoxybenzene as an internal standard. ${ }^{b}$ Isolated yields are in parentheses. ${ }^{c} \mathrm{HBF}_{4}$ was used as the proton source.

conversion and cleaner reaction were observed for $p$-tosic acid, and so this was used for further optimization. An increase in temperature to $60{ }^{\circ} \mathrm{C}$ resulted in a higher conversion and a much shorter reaction time (entry 4 ). Increasing both the palladium acetate catalyst loading to $15 \mathrm{~mol} \%$ and the reaction time to $1.5 \mathrm{~h}$ allowed full conversion (entry 5). Finally, lowering the catalyst loading to $5 \mathrm{~mol} \%$ under the optimized conditions still gave full conversion and an isolated yield of $68 \%$ for cinnamate $2 \mathrm{a}$. Throughout this optimization study and in accordance with the Felpin one-pot process, ${ }^{8 \mathrm{c}}$ no issues of dediazonization and decomposition of the aryl diazonium tosylate salt intermediate were observed. It should also be noted that during the early stages of optimization of this process, a wide range of palladium catalysts (e.g., $\mathrm{PdCl}_{2}$, $\mathrm{Pd}\left(\mathrm{PPh}_{3}\right)_{4}, \quad \mathrm{Pd}(\mathrm{tfa})_{2}, \quad \mathrm{Pd}(\text { hfacac })_{2}, \quad \mathrm{Pd}(\mathrm{acac})_{2}, \quad \mathrm{Pd}_{2} \mathrm{dba}_{3}$, $\mathrm{PdCl}_{2}(\mathrm{dppf})$ etc.) and associated ligands (e.g., $(n-\mathrm{Bu})_{3} \mathrm{P},(2-$ furyl $)_{3} \mathrm{P}, \mathrm{S}$-Phos, Dave-Phos, X-Phos, etc.) were trialed; however, the standard base-free conditions using palladium acetate gave the best results.

Following the optimization of a rapid and mild one-pot tandem process for diazotization and Heck coupling, the scope of this transformation was explored using various anilines and methyl acrylate (Scheme 2). Irrespective of the electronic nature or the substitution pattern of the aniline, all examples investigated were fully converted under the standard conditions to the corresponding Heck adduct in yields of 53-83\%. Interestingly, the one-pot diazotization and HeckMatsuda coupling of halogenated anilines was completely chemoselective, giving compounds $\mathbf{2} \mathbf{d}-\mathbf{2 f}, \mathbf{2} \mathbf{i}$, and $\mathbf{2 l}$ as the sole products. These results, particularly for $2 \mathbf{e}, \mathbf{2} \mathbf{f}$, and $\mathbf{2 i}$, exemplify the superior electrophilic nature of diazonium salts compared to halides. The one-pot process was also investigated for the synthesis of E-stilbenes. Reaction of 4nitroaniline (1a) with styrene or 4-fluorostyrene gave $E$ stilbenes $2 \mathrm{n}$ and $2 \mathrm{o}$ in 74 and $46 \%$ yields, respectively.

As the one-pot process with halogenated anilines gave only Heck-Matsuda adducts and none of the Mizoroki-Heck products, we wanted to exploit this chemoselectivity for the two-pot synthesis of orthogonally functionalized aryl com- 
Scheme 2. Scope of One-Pot Tandem Diazotization and Heck-Matsuda Process ${ }^{a}$

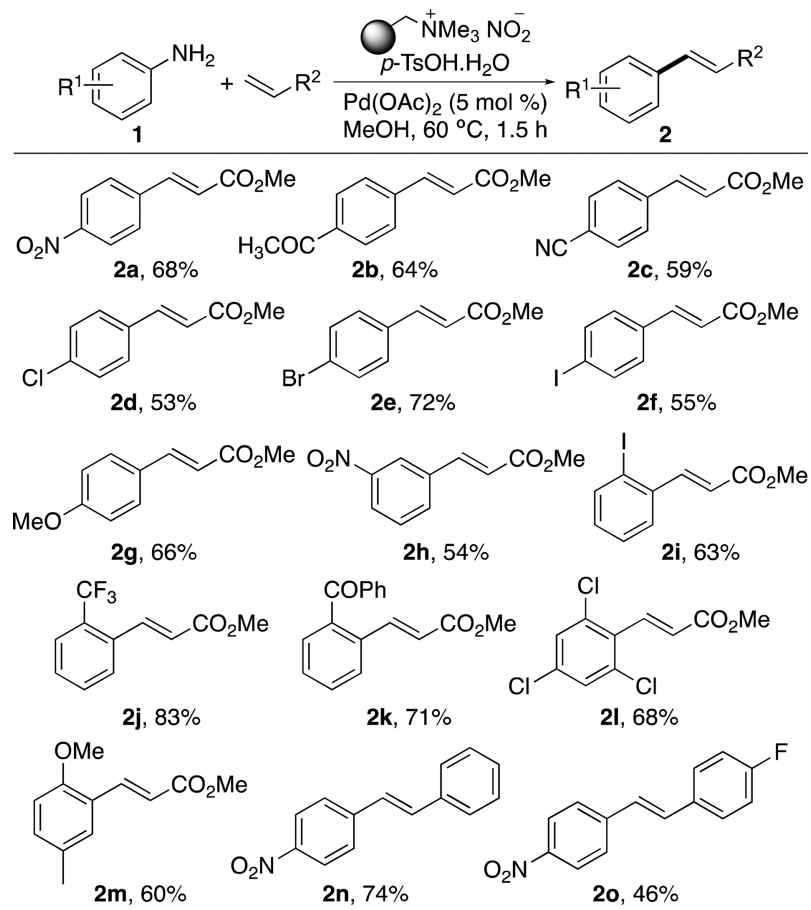

${ }^{a}$ Isolated yields are shown.

pounds. ${ }^{13}$ The one-pot diazotization and Heck-Matsuda coupling of 4-bromoaniline (1e) was scaled up, allowing the multigram synthesis of methyl (E)-3-(4-bromophenyl)acrylate (2e) (Scheme 3). Having used the amino group to implement the first cross-coupling process, $2 \mathrm{e}$ was then subjected to a Mizoroki-Heck reaction with styrene. ${ }^{14}$ This gave E-stilbene 3 in $72 \%$ yield. In a similar fashion, arylation or allylation by Suzuki-Miyaura reaction of $2 \mathrm{e}$ completed the efficient two-pot synthesis of acrylates $\mathbf{4 a - 4 d}$. This study shows that a combination of the one-pot diazotization and Heck-Matsuda process with a further cross-coupling reaction allows the highly controlled and selective introduction of multiple unsaturated moieties onto a central arene core.

A key objective of this project was to expand the scope of the one-pot diazotization and Heck-Matsuda process to include 2-nitroanilines as substrates. It was proposed that the resulting 2-nitrophenyl acrylates could be converted to 3,4dihydroquinolin-2-ones by extending the one-pot process to include reduction and cyclization steps. Before attempting the extended one-pot process, a series of substituted 2-nitroanilines was initially examined as substrates for the one-pot twostep tandem process (Scheme 4). Using the previously developed standard conditions, a wide range of acrylates $\mathbf{6 a -}$ 6n bearing various functional groups and substitution patterns were prepared in $56-87 \%$ yields. Again, complete chemoselectivity was observed with brominated $(\mathbf{5 i})$ and iodinated (5j) anilines.

As 2-nitroanilines were found to be excellent substrates for the one-pot diazotization and Heck-Matsuda reaction, the use of these in an extended one-pot multibond forming process for the preparation of 3,4-dihydroquinolin-2-ones was next investigated. 3,4-Dihydroquinolin-2-ones are important heterocyclic scaffolds for synthesis and are found in a wide range of pharmaceutically active agents. ${ }^{15}$ Because of this importance,
Scheme 3. Orthogonal Chemoselective Functionalization of 4-Bromoaniline $(1 \mathrm{e})^{a}$

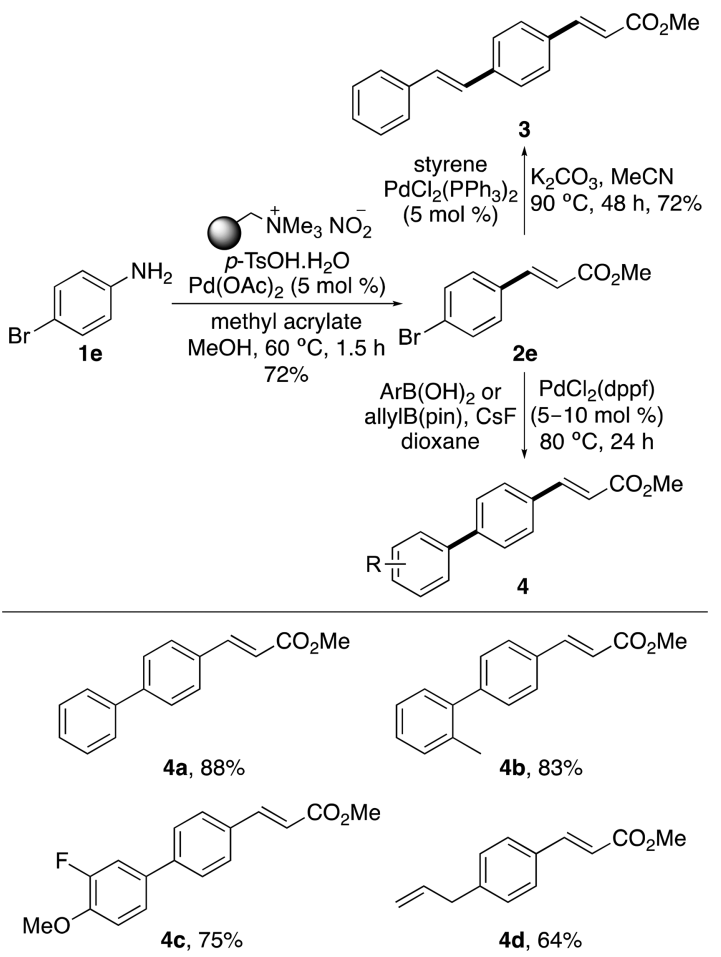

${ }^{a}$ Isolated yields are shown.

Scheme 4. Scope of One-Pot Tandem Diazotization and Heck-Matsuda Process using 2-Nitroanilines ${ }^{a}$

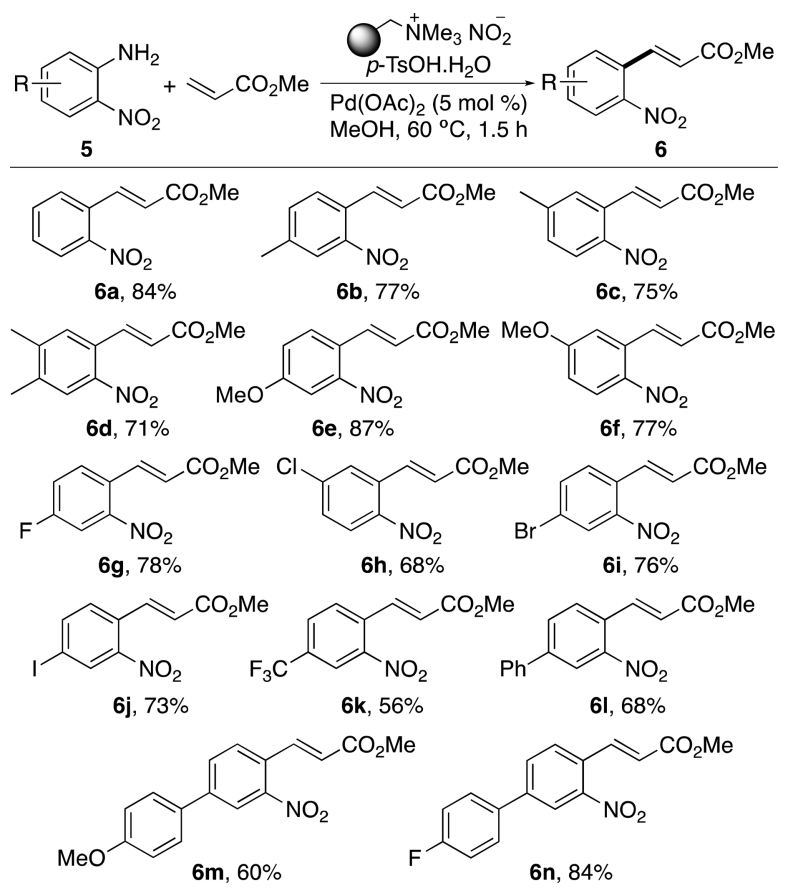

${ }^{a}$ Isolated yields are shown.

numerous methods have been developed for their synthesis, including one-pot processes. For example, Jiao and coworkers used a combination of radical and ionic processes for the reaction of 2 -iodoanilines with ethyl acrylate for the preparation of 3,4 -dihydroquinolin-2-ones in $17-71 \%$ yields, ${ }^{16}$ 
while the Lautens groups developed a one-pot $\mathrm{Rh} / \mathrm{Pd}$ catalyzed conjugate addition and amidation process of 2chlorophenyl boronic acids with acrylamide to generate a series of these compounds in 53-96\% yields. ${ }^{17}$ Felpin and coworkers also reported a one-pot synthesis of 3,4-dihydroquinolin-2ones from 2-nitroaryl tetrafluoroborate diazonium salts using a Heck-Matsuda reaction with acrylates followed by the addition of charcoal and a reduction and cyclization sequence. $^{8 \mathrm{~b}}$ In our study, we wanted to demonstrate that 3,4-dihydroquinolin-2-ones could be prepared directly from 2nitroanilines using the palladium catalyst to effect the HeckMatsuda coupling and the hydrogenation/reduction steps without the use of any additives. Using 2-nitroaniline (5a), the one-pot tandem diazotization and Heck-Matsuda sequence was performed as before (Scheme 5). On complete conversion

Scheme 5. One-Pot Synthesis of 3,4-Dihydroquinolin-2ones Using 2-Nitroanilines ${ }^{a}$

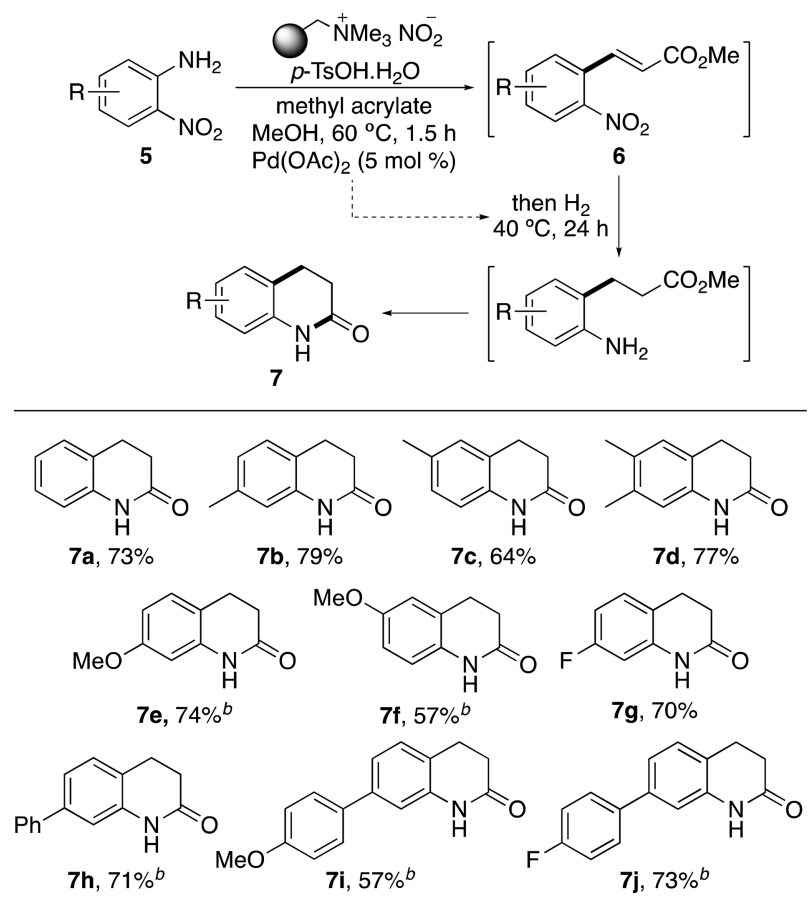

${ }^{a}$ Isolated yields are shown ${ }^{b}$ Reduction stage was conducted under a hydrogen atmosphere of $2.5 \mathrm{bar}$ and at room temperature.

to methyl acrylate $\mathbf{6 a}$, the reaction mixture was placed under an atmosphere of hydrogen. After $24 \mathrm{~h}$, this gave 3,4dihydroquinolin-2-one $7 a$ in $73 \%$ overall yield. Having shown that a one-pot diazotization/Heck-Matsuda/reduction/cyclization sequence could lead directly to 3,4-dihydroquinolin-2-ones, the scope and limitations of this process were investigated. Alkyl substituted 2-nitroanilines and a substrate bearing a fluorine substituent were readily converted to the corresponding 3,4-dihydroquinolin-2-ones $7 \mathbf{a}-7 \mathbf{d}$ and $7 \mathrm{~g}$, in yields of $64-79 \%$, under these conditions. Electron-rich methoxy substituted 2-nitroanilines 5 e and $\mathbf{5 f}$ could also be converted to 3,4-dihydroquinolin-2-ones $7 \mathbf{e}$ and $7 \mathbf{f}$, however, the hydrogenation/reduction step at atmospheric pressure was slow, leading to mixtures of the target 3,4-dihydroquinolin-2ones and methyl 2-nitrophenylpropionate intermediates after $24 \mathrm{~h}^{18}$ To effect cleaner, more efficient syntheses of these compounds, the one-pot process was repeated by conducting the reduction stage under pressure at 2.5 bar. This gave 3,4dihydroquinolin-2-ones $7 \mathbf{e}$ and $7 \mathbf{f}$ as the sole products in 74 and $57 \%$ yields, respectively. A limitation of this one-pot process is that 2-nitroanilines bearing labile carbon-halogen bonds are subject to dehalogenation at the reduction stage. For example, the attempted use of 5-chloro-2-nitroaniline $(\mathbf{5 h})$ as a substrate for this process gave 5-chloro-3,4-dihydroquinolin-2one in $28 \%$ yield but as a 1:1 inseparable mixture with the deschlorinated product $7 \mathbf{a}$. Our interest in preparing halogenated 3,4-dihydroquinolin-2-ones was for the potential structural diversification of these through cross-coupling reactions after the one-pot process. To overcome this limitation, crosscoupling reactions were conducted prior to the one-pot process for the efficient two-pot synthesis of 7-aryl-3,4dihydroquinolin-2-ones. Suzuki-Miyaura reaction of 4-iodo2-nitroaniline $(\mathbf{5 j})$ with various aryl boronic acids (see Supporting Information for full details) was then followed by the one-pot process, which gave $7 \mathbf{h}-7 \mathbf{j}$ in $57-73 \%$ yield. While the standard diazotization and Heck-Matsuda steps for the 4-aryl-2-nitroanilines could be conducted under standard conditions, again the use of 2.5 bar of pressure for the reduction stage allowed the most efficient synthesis of these analogues.

The synthetic potential of the 3,4-dihydroquinolin-2-ones prepared from the extended one-pot process was then demonstrated with the synthesis of a pharmaceutically active target. $\mathrm{N}$-Acetic acid derived 3,4-dihydroquinolin-2-ones bearing 6- or 7-aryl groups are late stage sodium channel blockers and have the potential to be used in the treatment of cardiovascular diseases and diabetes. ${ }^{19}$ In this study, a sodium channel modulator, 3,4-dihydroquinolin-2-one 11, was prepared via a five-pot synthesis (Scheme 6). As shown above, the extended one-pot process was used to convert 5-methoxy-2nitroaniline (5e) to the corresponding 3,4-dihydroquinolin-2-

Scheme 6. Five-Pot Synthesis of Quinolinone Based Sodium Ion Channel Modulator $11^{a}$

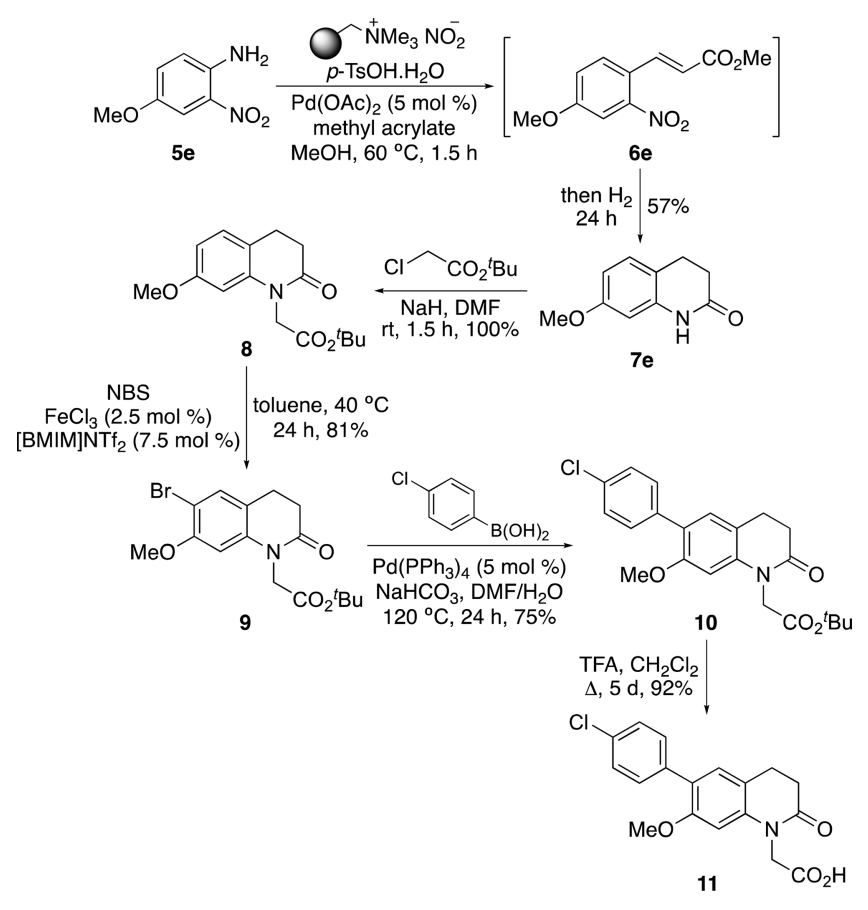

${ }^{a}$ Isolated yields are shown. 
one $7 \mathbf{e}$ on a gram scale in $57 \%$ yield. Quinolin-2-one $7 \mathbf{e}$ was alkylated in quantitative yield using $t$-butyl chloroacetate and sodium hydride. A highly regioselective iron(III) triflimidecatalyzed halogenation with $N$-bromosuccinimide (NBS) was used to functionalize the 6-position of the aryl ring. ${ }^{20}$ The combination of catalytic amounts of iron(III) chloride and the ionic liquid [BMIM] $\mathrm{NTf}_{2}$ forms iron(III) triflimide in situ, which acts as a powerful Lewis acid for the activation of NBS. This reaction gave 6-bromo-3,4-dihydroquinolin-2-one 9 as the sole product in $81 \%$ yield. Suzuki-Miyaura coupling of 9 with 4-chlorophenyl boronic acid under standard conditions gave 6aryl-3,4-dihydroquinolin-2-one $\mathbf{1 0}$ in $75 \%$ yield. Finally, TFA mediated deprotection of the $t$-butyl ester completed the synthesis of sodium ion channel modulator 11 in $32 \%$ overall yield from 2-nitroaniline 5 e.

\section{CONCLUSIONS}

In summary, a rapid one-pot tandem process involving diazotization and base-free Heck-Matsuda coupling of anilines with acrylates and styrenes was developed. The use of a particularly mild procedure to form stable aryl diazonium tosylate salts using a polymer-supported nitrite reagent and $p$ tosic acid as the proton source allowed the synthesis of a wide range of Heck adducts. A particular feature of this process was the chemoselective coupling of brominated and iodinated anilines, which could then be further functionalized using additional cross-coupling reactions, leading to orthogonally substituted arenes. Using 2-nitroanilines as substrates, the onepot process was extended for the direct synthesis of 3,4dihydroquinolin-2-ones. Following the diazotization and Heck-Matsuda steps, reutilization of the palladium catalyst without the requirement of additional ligands or additives allowed hydrogenation of the alkene, reduction of the nitro group, and in situ cyclization to give a series of 3,4dihydroquinolin-2-ones. The potential of these one-pot multibond forming processes for application in total synthesis and medicinal chemistry was demonstrated with the five-pot preparation of a sodium ion channel modulator. Investigation of further applications of stable aryl diazonium salts in one-pot multistep processes is currently underway.

\section{EXPERIMENTAL SECTION}

All reagents and starting materials were obtained from commercial sources and used as received unless otherwise stated. Dry solvents were purified using a solvent purification system. Brine refers to a saturated solution of sodium chloride. All reactions were performed in oven-dried glassware under an atmosphere of argon unless otherwise stated. Flash column chromatography was carried out using silica gel $(40-63 \mu \mathrm{m})$ and neutral aluminum oxide $(50-200 \mu \mathrm{m})$. Aluminumbacked plates precoated with silica gel $60\left(\mathrm{UV}_{254}\right)$ were used for thin layer chromatography and were visualized under ultraviolet light and by staining with $\mathrm{KMnO}_{4}$ or ninhydrin. ${ }^{1} \mathrm{H}$ NMR spectra were recorded on a NMR spectrometer at $400 \mathrm{MHz}$ and data are reported as follows: chemical shift in ppm relative to tetramethylsilane or the solvent as the internal standard $\left(\mathrm{CDCl}_{3}, \delta 7.26 \mathrm{ppm}\right)$, multiplicity (s = singlet, $\mathrm{d}=$ doublet, $\mathrm{t}=$ triplet, $\mathrm{q}=$ quartet, $\mathrm{m}=$ multiplet or overlap of nonequivalent resonances, integration). ${ }^{13} \mathrm{C}$ NMR spectra were recorded on a NMR spectrometer at $101 \mathrm{MHz}$ and data are reported as follows: chemical shift in ppm relative to tetramethylsilane or the solvent as internal standard $\left(\mathrm{CDCl}_{3}, \delta 77.0 \mathrm{ppm}\right)$, multiplicity with respect to hydrogen (deduced from DEPT experiments, $\mathrm{C}, \mathrm{CH}, \mathrm{CH}_{2}$, or $\mathrm{CH}_{3}$ ). IR spectra were recorded on a FTIR spectrometer; wavenumbers are indicated in $\mathrm{cm}^{-1}$. Mass spectra were recorded using electron impact or electrospray techniques. HRMS spectra were recorded using a dual-focusing magnetic analyzer mass spectrometer. Melting points are uncorrected.

General Procedure for the Preparation of the PolymerSupported Nitrite. To a stirred solution of sodium nitrite $(0.55 \mathrm{~g}$, $8.00 \mathrm{mmol})$ in water $(20 \mathrm{~mL})$ was added Amberlyst A26 hydroxide form resin $(1.00 \mathrm{~g}, 4.00 \mathrm{mmol})$. The resulting mixture was stirred at room temperature for $0.5 \mathrm{~h}$, and then polymer-supported resin was filtered and washed with water until the $\mathrm{pH}$ of filtrate became neutral. The content of prepared polymer-supported nitrite was $3.5 \mathrm{mmol}$ of $\mathrm{NO}_{2}$ per $\mathrm{g}$ of resin. 9

Methyl (E)-3-(4'-Nitrophenyl)acrylate $(2 a){ }^{8 c}$ To a stirred solution of 4-nitroaniline $(1 \mathrm{a})(0.028 \mathrm{~g}, 0.20 \mathrm{mmol})$, polymer-supported nitrite $\left(0.17 \mathrm{~g}\right.$, containing $0.60 \mathrm{mmol}$ of $\left.\mathrm{NO}_{2}\right), p$-toluenesulfonic acid monohydrate $(0.10 \mathrm{~g}, 0.60 \mathrm{mmol})$, and palladium(II) acetate $(0.0020$ $\mathrm{g}, 0.010 \mathrm{mmol})$ in methanol $(2 \mathrm{~mL})$ was added methyl acrylate $(0.090$ $\mathrm{mL}, 1.0 \mathrm{mmol}$ ). The reaction mixture was heated to $60^{\circ} \mathrm{C}$ and stirred for $1.5 \mathrm{~h}$. The mixture was cooled to room temperature and filtered, and the resin was washed with methanol $(2 \mathrm{~mL})$. The combined organic layers were concentrated in vacuo. Purification by silica gel flash column chromatography, eluting with $10 \%$ diethyl ether in hexane gave methyl (E)-3-(4'-nitrophenyl)acrylate (2a) (0.028 g, $68 \%$ ) as a pale yellow solid. Mp $159-161{ }^{\circ} \mathrm{C}\left(\right.$ lit. $\left.^{8 \mathrm{c}} 161{ }^{\circ} \mathrm{C}\right) ;{ }^{1} \mathrm{H}$ NMR $\left(400 \mathrm{MHz}, \mathrm{CDCl}_{3}\right) \delta 3.83(\mathrm{~s}, 3 \mathrm{H}), 6.56(\mathrm{~d}, J=16.1 \mathrm{~Hz}, 1 \mathrm{H})$, $7.67(\mathrm{~d}, J=8.7 \mathrm{~Hz}, 2 \mathrm{H}), 7.71(\mathrm{~d}, J=16.1 \mathrm{~Hz}, 1 \mathrm{H}), 8.24(\mathrm{~d}, J=8.7$ $\mathrm{Hz}, 2 \mathrm{H}) ;{ }^{13} \mathrm{C}$ NMR $\left(101 \mathrm{MHz}, \mathrm{CDCl}_{3}\right) \delta 52.1\left(\mathrm{CH}_{3}\right), 122.1(\mathrm{CH})$, $124.2(2 \times \mathrm{CH}), 128.7(2 \times \mathrm{CH}), 140.5(\mathrm{C}), 141.9(\mathrm{CH}), 148.6(\mathrm{C})$, 166.5 (C); MS (EI) m/z $207\left(\mathrm{M}^{+}, 66\right), 176$ (100), 130 (24), 102 (26).

Methyl (E)-3-(4'-Acetylphenyl)acrylate (2b). ${ }^{21}$ The reaction was carried out according to the previously described procedure for methyl (E)-3-(4'-nitrophenyl)acrylate (2a) using 4-aminoacetophenone $(\mathbf{1 b})(0.050 \mathrm{~g}, 0.37 \mathrm{mmol})$, polymer-supported nitrite $(0.32 \mathrm{~g}$, containing $1.1 \mathrm{mmol}$ of $\mathrm{NO}_{2}$ ), $p$-toluenesulfonic acid monohydrate $(0.19 \mathrm{~g}, 1.1 \mathrm{mmol})$, palladium(II) acetate $(0.0040 \mathrm{~g}, 0.019 \mathrm{mmol})$, and methyl acrylate $(0.17 \mathrm{~mL}, 1.9 \mathrm{mmol})$. Purification by silica gel flash column chromatography, eluting with $10 \%$ diethyl ether in hexane gave methyl (E)-3-(4'-acetylphenyl)acrylate (2b) (0.050 g, $64 \%)$ as an orange oil. Spectroscopic data were consistent with the literature. ${ }^{21}{ }^{1} \mathrm{H}$ NMR $\left(400 \mathrm{MHz}, \mathrm{CDCl}_{3}\right) \delta 2.62(\mathrm{~s}, 3 \mathrm{H}), 3.83(\mathrm{~s}$, $3 \mathrm{H}), 6.53(\mathrm{~d}, J=16.1 \mathrm{~Hz}, 1 \mathrm{H}), 7.61(\mathrm{~d}, J=8.3 \mathrm{~Hz}, 2 \mathrm{H}), 7.71(\mathrm{~d}, J=$ $16.1 \mathrm{~Hz}, 1 \mathrm{H}), 7.97(\mathrm{~d}, J=8.3 \mathrm{~Hz}, 2 \mathrm{H}) ;{ }^{13} \mathrm{C} \mathrm{NMR}\left(101 \mathrm{MHz}, \mathrm{CDCl}_{3}\right)$ $\delta 26.6\left(\mathrm{CH}_{3}\right), 51.9\left(\mathrm{CH}_{3}\right), 120.3(\mathrm{CH}), 128.1(2 \times \mathrm{CH}), 128.8(2 \times$ $\mathrm{CH}), 138.1$ (C), 138.7 (C), $143.3(\mathrm{CH}), 166.9$ (C), $197.2(\mathrm{C})$; MS (ESI) $m / z 205\left(\mathrm{M}+\mathrm{H}^{+}, 100\right)$.

Methyl (E)-3-(4'-Cyanophenyl)acrylate (2C). ${ }^{22}$ The reaction was carried out according to the previously described procedure for methyl (E)-3-(4'-nitrophenyl)acrylate (2a) using 4-aminobenzonitrile (1c) $(0.050 \mathrm{~g}, 0.42 \mathrm{mmol})$, polymer-supported nitrite $(0.36 \mathrm{~g}$, containing $1.3 \mathrm{mmol}$ of $\mathrm{NO}_{2}$ ), $p$-toluenesulfonic acid monohydrate $(0.22 \mathrm{~g}, 1.3 \mathrm{mmol})$, palladium(II) acetate $(0.005 \mathrm{~g}, 0.02 \mathrm{mmol})$, and methyl acrylate $(0.19 \mathrm{~mL}, 2.1 \mathrm{mmol})$. Purification by neutral alumina and then silica gel flash column chromatography, eluting with $10 \%$ diethyl ether in hexane gave methyl $(E)-3-\left(4^{\prime}\right.$-cyanophenyl)acrylate (2c) $(0.046 \mathrm{~g}, 59 \%)$ as a yellow solid. Mp $120-122^{\circ} \mathrm{C}$ (lit. ${ }^{22} 121.7-$ $\left.122.1{ }^{\circ} \mathrm{C}\right) ;{ }^{1} \mathrm{H}$ NMR $\left(400 \mathrm{MHz}, \mathrm{CDCl}_{3}\right) \delta 3.83(\mathrm{~s}, 3 \mathrm{H}), 6.52(\mathrm{~d}, J=$ $16.0 \mathrm{~Hz}, 1 \mathrm{H}), 7.61(\mathrm{~d}, J=8.3 \mathrm{~Hz}, 2 \mathrm{H}), 7.64-7.71(\mathrm{~m}, 3 \mathrm{H}) ;{ }^{13} \mathrm{C}$ NMR $\left(101 \mathrm{MHz}, \mathrm{CDCl}_{3}\right) \delta 52.1\left(\mathrm{CH}_{3}\right), 113.5(\mathrm{C}), 118.4(\mathrm{C}), 121.4$ $(\mathrm{CH}), 128.4(2 \times \mathrm{CH}), 132.7(2 \times \mathrm{CH}), 138.7(\mathrm{C}), 142.4(\mathrm{CH})$, 166.6 (C); MS (EI) $m / z 187\left(\mathrm{M}^{+}, 50\right), 156$ (100), 128 (42), 84 (27).

Methyl (E)-3-(4'-Chlorophenyl)acrylate (2d). ${ }^{8 d}$ The reaction was carried out according to the previously described procedure for methyl (E)-3-(4'-nitrophenyl)acrylate (2a) using 4-chloroaniline (1d) $(0.050 \mathrm{~g}, 0.39 \mathrm{mmol})$, polymer-supported nitrite $(0.33 \mathrm{~g}$, containing $1.2 \mathrm{mmol}$ of $\mathrm{NO}_{2}$ ), $p$-toluenesulfonic acid monohydrate $(0.20 \mathrm{~g}, 1.2 \mathrm{mmol})$, palladium(II) acetate $(0.0040 \mathrm{~g}, 0.020 \mathrm{mmol})$, and methyl acrylate $(0.18 \mathrm{~mL}, 2.0 \mathrm{mmol})$. Purification by neutral alumina and then silica gel flash column chromatography, eluting with $10 \%$ diethyl ether in hexane gave methyl $(E)-3-\left(4^{\prime}\right.$-chlorophenyl)acrylate $(\mathbf{2 d})(0.040 \mathrm{~g}, 53 \%)$ as a yellow solid. $\mathrm{Mp} 73-75^{\circ} \mathrm{C}$ (lit. ${ }^{8 \mathrm{~d}}$ $\left.74-75^{\circ} \mathrm{C}\right) ;{ }^{1} \mathrm{H}$ NMR $\left(400 \mathrm{MHz}, \mathrm{CDCl}_{3}\right) \delta 3.80(\mathrm{~s}, 3 \mathrm{H}), 6.41(\mathrm{~d}, J=$ 
$16.0 \mathrm{~Hz}, 1 \mathrm{H}), 7.35$ (d, $J=8.5 \mathrm{~Hz}, 2 \mathrm{H}), 7.45$ (d, $J=8.5 \mathrm{~Hz}, 2 \mathrm{H}), 7.63$ $(\mathrm{d}, J=16.0 \mathrm{~Hz}, 2 \mathrm{H}) ;{ }^{13} \mathrm{C} \mathrm{NMR}\left(101 \mathrm{MHz}, \mathrm{CDCl}_{3}\right) \delta 51.8\left(\mathrm{CH}_{3}\right)$, $118.4(\mathrm{CH}), 129.2(2 \times \mathrm{CH}), 129.2(2 \times \mathrm{CH}), 132.9(\mathrm{C}), 136.2(\mathrm{C})$, $143.4(\mathrm{CH}), 167.2(\mathrm{C})$; MS (EI) $m / z 196\left(\mathrm{M}^{+}, 68\right), 165$ (100), 137 (27), 101 (24), 78 (22), 63 (25).

Methyl (E)-3-(4'-Bromophenyl)acrylate (2e). ${ }^{22}$ The reaction was carried out according to the previously described procedure for methyl (E)-3-(4'-nitrophenyl)acrylate (2a) using 4-bromoaniline (1e) $(0.050 \mathrm{~g}, 0.29 \mathrm{mmol})$, polymer-supported nitrite $(0.25 \mathrm{~g}$, containing $0.87 \mathrm{mmol}$ of $\mathrm{NO}_{2}$ ), p-toluenesulfonic acid monohydrate $(0.15 \mathrm{~g}, 0.87 \mathrm{mmol})$, palladium(II) acetate $(0.0030 \mathrm{~g}, 0.015 \mathrm{mmol})$, and methyl acrylate $(0.13 \mathrm{~mL}, 1.5 \mathrm{mmol})$. Purification by neutral alumina flash column chromatography, eluting with $10 \%$ diethyl ether in hexane gave methyl (E)-3-( $4^{\prime}$-bromophenyl)acrylate (2e) $(0.050 \mathrm{~g}$, $72 \%)$ as a yellow solid. $\mathrm{Mp} 86-88{ }^{\circ} \mathrm{C}\left(\right.$ lit. $\left.^{22} 87.9-88.2{ }^{\circ} \mathrm{C}\right) ;{ }^{1} \mathrm{H}$ NMR $\left(400 \mathrm{MHz}, \mathrm{CDCl}_{3}\right) \delta 3.81(\mathrm{~s}, 3 \mathrm{H}), 6.43(\mathrm{~d}, J=16.0 \mathrm{~Hz}, 1 \mathrm{H}), 7.38(\mathrm{~d}$, $J=8.5 \mathrm{~Hz}, 2 \mathrm{H}), 7.52(\mathrm{~d}, J=8.5 \mathrm{~Hz}, 2 \mathrm{H}), 7.62(\mathrm{~d}, J=16.0 \mathrm{~Hz}, 1 \mathrm{H})$; ${ }^{13} \mathrm{C} \mathrm{NMR}\left(101 \mathrm{MHz}, \mathrm{CDCl}_{3}\right) \delta 51.8\left(\mathrm{CH}_{3}\right), 118.5(\mathrm{CH}), 124.6(\mathrm{C})$, $129.5(2 \times \mathrm{CH}), 132.2(2 \times \mathrm{CH}), 133.3(\mathrm{C}), 143.5(\mathrm{CH}), 167.2(\mathrm{C})$; MS (EI) $m / z 242\left(\mathrm{M}^{+}, 95\right), 240$ (96), 211 (99), 209 (100), 102 (87), $84(55)$.

Methyl (E)-3-(4'-lodophenyl)acrylate (2f). ${ }^{22}$ The reaction was carried out according to the previously described procedure for methyl (E)-3-(4'-nitrophenyl)acrylate (2a) using 4-iodoaniline (1f) $(0.050 \mathrm{~g}, 0.23 \mathrm{mmol})$, polymer-supported nitrite $(0.20 \mathrm{~g}$, containing $0.69 \mathrm{mmol}$ of $\left.\mathrm{NO}_{2}\right), p$-toluenesulfonic acid monohydrate $(0.12 \mathrm{~g}, 0.69$ $\mathrm{mmol})$, palladium(II) acetate $(0.0030 \mathrm{~g}, 0.012 \mathrm{mmol})$, and methyl acrylate $(0.10 \mathrm{~mL}, 1.2 \mathrm{mmol})$. Purification by alumina flash column chromatography, eluting with $2 \%$ diethyl ether in hexane gave methyl (E)-3-(4'-iodophenyl)acrylate (2f) $(0.036 \mathrm{~g}, 55 \%)$ as a white solid. Mp $117-119{ }^{\circ} \mathrm{C}$ (lit. $\left.{ }^{22} 119-123.9{ }^{\circ} \mathrm{C}\right) ;{ }^{1} \mathrm{H}$ NMR $(400 \mathrm{MHz}$, $\left.\mathrm{CDCl}_{3}\right) \delta 3.80(\mathrm{~s}, 3 \mathrm{H}), 6.44(\mathrm{~d}, J=16.0 \mathrm{~Hz}, 1 \mathrm{H}), 7.24(\mathrm{~d}, J=8.4 \mathrm{~Hz}$, $2 \mathrm{H}), 7.60(\mathrm{~d}, J=16.0 \mathrm{~Hz}, 1 \mathrm{H}), 7.73(\mathrm{~d}, J=8.4 \mathrm{~Hz}, 2 \mathrm{H}) ;{ }^{13} \mathrm{C} \mathrm{NMR}$ $\left(101 \mathrm{MHz}, \mathrm{CDCl}_{3}\right) \delta 51.8\left(\mathrm{CH}_{3}\right), 96.5(\mathrm{C}), 118.6(\mathrm{CH}), 129.5(2 \times$ $\mathrm{CH}), 133.9(\mathrm{C}), 138.1(2 \times \mathrm{CH}), 143.7(\mathrm{CH}), 167.2(\mathrm{C})$; MS (EI) $\mathrm{m} / z 288$ ( $\left.\mathrm{M}^{+}, 96\right), 257$ (40), 130 (27), 102 (20), 84 (100).

Methyl (E)-3-(4'-Methoxyphenyl)acrylate $(\mathbf{2 g})$. $^{23}$ The reaction was carried out according to the previously described procedure for methyl (E)-3-(4'-nitrophenyl)acrylate (2a) using 4-methoxyaniline (1g) $(0.050 \mathrm{~g}, 0.41 \mathrm{mmol})$, polymer-supported nitrite $(0.35 \mathrm{~g}$, containing $1.2 \mathrm{mmol}$ of $\mathrm{NO}_{2}$ ), $p$-toluenesulfonic acid monohydrate $(0.21 \mathrm{~g}, 1.2 \mathrm{mmol})$, palladium(II) acetate $(0.0050 \mathrm{~g}, 0.021 \mathrm{mmol})$, and methyl acrylate $(0.18 \mathrm{~mL}, 2.1 \mathrm{mmol})$. Purification by neutral alumina flash column chromatography, eluting with $20 \%$ diethyl ether in hexane gave methyl $(E)-3-\left(4^{\prime}\right.$-methoxyphenyl)acrylate $(2 \mathrm{~g})(0.052$ g, 66\%) as a white solid. Mp $85-87^{\circ} \mathrm{C}\left(\right.$ lit. $\left.^{23} 86^{\circ} \mathrm{C}\right) ;{ }^{1} \mathrm{H}$ NMR $(400$ $\left.\mathrm{MHz}, \mathrm{CDCl}_{3}\right) \delta 3.79(\mathrm{~s}, 3 \mathrm{H}), 3.84(\mathrm{~s}, 3 \mathrm{H}), 6.31(\mathrm{~d}, J=16.0 \mathrm{~Hz}, 1 \mathrm{H})$, $6.90(\mathrm{~d}, J=8.7 \mathrm{~Hz}, 2 \mathrm{H}), 7.47(\mathrm{~d}, J=8.7 \mathrm{~Hz}, 2 \mathrm{H}), 7.65(\mathrm{~d}, J=16.0$ $\mathrm{Hz}, 1 \mathrm{H}) ;{ }^{13} \mathrm{C} \mathrm{NMR}\left(101 \mathrm{MHz}, \mathrm{CDCl}_{3}\right) \delta 51.6\left(\mathrm{CH}_{3}\right), 55.4\left(\mathrm{CH}_{3}\right)$, $114.3(2 \times \mathrm{CH}), 115.3(\mathrm{CH}), 127.2(\mathrm{C}), 129.7(2 \times \mathrm{CH}), 144.5$ (CH), 161.4 (C), 167.8 (C); MS (ESI) $m / z 193\left(\mathrm{M}+\mathrm{H}^{+}, 100\right)$.

Methyl (E)-3-(3'-Nitrophenyl)acrylate (2h). ${ }^{24}$ The reaction was carried out according to the previously described procedure for methyl (E)-3-(4'-nitrophenyl)acrylate (2a) using 3-nitroaniline (1h) $(0.050 \mathrm{~g}, 0.36 \mathrm{mmol})$, polymer-supported nitrite $(0.31 \mathrm{~g}$, containing $1.1 \mathrm{mmol}$ of $\left.\mathrm{NO}_{2}\right), p$-toluenesulfonic acid monohydrate $(0.19 \mathrm{~g}, 1.1$ $\mathrm{mmol})$, palladium(II) acetate $(0.0040 \mathrm{~g}, 0.018 \mathrm{mmol})$, and methyl acrylate $(0.16 \mathrm{~mL}, 1.8 \mathrm{mmol})$. Purification by neutral alumina flash column chromatography, eluting with $5 \%$ diethyl ether in hexane gave methyl (E)-3-(3'-nitrophenyl)acrylate (2h) $(0.040 \mathrm{~g}, 54 \%)$ as a pale yellow solid. Mp $120-122{ }^{\circ} \mathrm{C}$ (lit. $\left.{ }^{24} 122-123{ }^{\circ} \mathrm{C}\right) ;{ }^{1} \mathrm{H}$ NMR $(400$ $\left.\mathrm{MHz}, \mathrm{CDCl}_{3}\right) \delta 3.83(\mathrm{~s}, 3 \mathrm{H}), 6.56(\mathrm{~d}, J=16.0 \mathrm{~Hz}, 1 \mathrm{H}), 7.58(\mathrm{t}, J=$ $8.0 \mathrm{~Hz}, 1 \mathrm{H}), 7.72(\mathrm{~d}, J=16.0 \mathrm{~Hz}, 1 \mathrm{H}), 7.82(\mathrm{br} \mathrm{d}, J=8.0 \mathrm{~Hz}, 1 \mathrm{H})$, 8.22 (ddd, $J=8.0,2.0,0.8 \mathrm{~Hz}, 1 \mathrm{H}), 8.37(\mathrm{t}, J=2.0 \mathrm{~Hz}, 1 \mathrm{H}) ;{ }^{13} \mathrm{C}$ NMR $\left(101 \mathrm{MHz}, \mathrm{CDCl}_{3}\right) \delta 52.0\left(\mathrm{CH}_{3}\right), 121.0(\mathrm{CH}), 122.5(\mathrm{CH})$, $124.6(\mathrm{CH}), 130.0(\mathrm{CH}), 133.6(\mathrm{CH}), 136.1(\mathrm{C}), 142.0(\mathrm{CH}), 148.7$ (C), 166.6 (C); MS (ESI) $m / z 230\left(\mathrm{M}+\mathrm{Na}^{+}, 100\right)$.

Methyl (E)-3-(2'-lodophenyl)acrylate (2i). ${ }^{25}$ The reaction was carried out according to the previously described procedure for methyl (E)-3-(4'-nitrophenyl)acrylate (2a) using 2-iodoaniline (1i)
$(0.050 \mathrm{~g}, 0.23 \mathrm{mmol})$, polymer-supported nitrite $(0.20 \mathrm{~g}$, containing $0.69 \mathrm{mmol}$ of $\left.\mathrm{NO}_{2}\right), p$-toluenesulfonic acid monohydrate $(0.12 \mathrm{~g}, 0.69$ $\mathrm{mmol})$, palladium(II) acetate $(0.0030 \mathrm{~g}, 0.011 \mathrm{mmol})$, and methyl acrylate $(0.10 \mathrm{~mL}, 1.2 \mathrm{mmol})$. Purification by neutral alumina and then silica gel flash column chromatography, eluting with $2 \%$ diethyl ether in hexane gave methyl (E)-3-(2'-iodophenyl)acrylate (2i) $(0.042 \mathrm{~g}, 63 \%)$ as a yellow oil. Spectroscopic data were consistent with the literature. ${ }^{25}{ }^{1} \mathrm{H}$ NMR $\left(400 \mathrm{MHz}, \mathrm{CDCl}_{3}\right) \delta 3.82(\mathrm{~s}, 3 \mathrm{H})$, $6.31(\mathrm{~d}, J=15.8 \mathrm{~Hz}, 1 \mathrm{H}), 7.05(\mathrm{td}, J=8.0,1.6 \mathrm{~Hz}, 1 \mathrm{H}), 7.35(\mathrm{td}, J=$ 8.0, $1.2 \mathrm{~Hz}, 1 \mathrm{H}), 7.55(\mathrm{dd}, J=8.0,1.6 \mathrm{~Hz}, 1 \mathrm{H}), 7.89(\mathrm{dd}, J=8.0,1.2$ $\mathrm{Hz}, 1 \mathrm{H}), 7.90(\mathrm{~d}, J=15.8 \mathrm{~Hz}, 1 \mathrm{H}) ; \delta_{\mathrm{C}}\left(101 \mathrm{MHz}, \mathrm{CDCl}_{3}\right) \delta 51.9$ $\left(\mathrm{CH}_{3}\right), 101.2(\mathrm{C}), 120.8(\mathrm{CH}), 127.4(\mathrm{CH}), 128.6(\mathrm{CH}), 131.3$ (CH), $137.8(\mathrm{C}), 140.0(\mathrm{CH}), 147.9(\mathrm{CH}), 166.7(\mathrm{C})$; MS (ESI) m/ $z 311\left(\mathrm{M}+\mathrm{Na}^{+}, 100\right)$.

Methyl (E)-3-[2'-(Trifluoromethyl)phenyl]acrylate $(2 j){ }^{26}$ The reaction was carried out according to the previously described procedure for methyl (E)-3-(4'-nitrophenyl)acrylate (2a) using 2trifluoromethylaniline $(\mathbf{1 j})(0.040 \mathrm{~mL}, 0.31 \mathrm{mmol})$, polymersupported nitrite $\left(0.27 \mathrm{~g}\right.$, containing $0.93 \mathrm{mmol}$ of $\left.\mathrm{NO}_{2}\right), p$ toluenesulfonic acid monohydrate $(0.16 \mathrm{~g}, 0.93 \mathrm{mmol})$, palladium(II) acetate $(0.0040 \mathrm{~g}, 0.016 \mathrm{mmol})$, and methyl acrylate $(0.14 \mathrm{~mL}, 1.6$ $\mathrm{mmol})$. Purification by neutral alumina and then silica gel flash column chromatography, eluting with $10 \%$ diethyl ether in hexane gave methyl (E)-3-[2'-(trifluoromethyl)phenyl] acrylate $(2 \mathbf{j})(0.059 \mathrm{~g}$, $83 \%)$ as a yellow oil. Spectroscopic data were consistent with the literature. ${ }^{26}{ }^{1} \mathrm{H} \mathrm{NMR}\left(400 \mathrm{MHz}, \mathrm{CDCl}_{3}\right) \delta 3.83(\mathrm{~s}, 3 \mathrm{H}), 6.41(\mathrm{~d}, J=$ $16.0 \mathrm{~Hz}, 1 \mathrm{H}), 7.48(\mathrm{t}, J=8.0 \mathrm{~Hz}, 1 \mathrm{H}), 7.57(\mathrm{t}, J=8.0 \mathrm{~Hz}, 1 \mathrm{H}), 7.70$ $($ br d, $J=8.0 \mathrm{~Hz}, 2 \mathrm{H}), 8.06(\mathrm{dq}, J=16.0,2.0 \mathrm{~Hz}, 1 \mathrm{H}) ;{ }^{13} \mathrm{C}$ NMR $(101$ $\left.\mathrm{MHz}, \mathrm{CDCl}_{3}\right) \delta 51.9\left(\mathrm{CH}_{3}\right), 122.2(\mathrm{CH}), 123.9\left(\mathrm{q},{ }^{1} J_{\mathrm{C}-\mathrm{F}}=274.0 \mathrm{~Hz}\right.$, C), $126.2\left(\mathrm{q},{ }^{3} J_{\mathrm{C}-\mathrm{F}}=5.6 \mathrm{~Hz}, \mathrm{CH}\right), 127.9(\mathrm{CH}), 128.9\left(\mathrm{q},{ }^{2} J_{\mathrm{C}-\mathrm{F}}=30.4\right.$ $\mathrm{Hz}, \mathrm{C}), 129.6(\mathrm{CH}), 132.1\left(\mathrm{q},{ }^{4} J_{\mathrm{C}-\mathrm{F}}=1.0 \mathrm{~Hz}, \mathrm{CH}\right), 133.4\left(\mathrm{q},{ }^{3} J_{\mathrm{C}-\mathrm{F}}=\right.$ $1.3 \mathrm{~Hz}, \mathrm{C}), 140.3\left(\mathrm{q},{ }^{4} J_{\mathrm{C}-\mathrm{F}}=1.9 \mathrm{~Hz}, \mathrm{CH}\right), 166.5(\mathrm{C}) ; \mathrm{MS}(\mathrm{ESI}) \mathrm{m} / \mathrm{z}$ $231\left(\mathrm{M}+\mathrm{H}^{+}, 100\right)$.

Methyl (E)-3-(2'-Benzoylphenyl)acrylate $(\mathbf{2 k}) .^{27}$ The reaction was carried out according to the previously described procedure for methyl (E)-3-(4'-nitrophenyl)acrylate (2a) using 2-aminobenzophenone $(1 \mathrm{k})(0.050 \mathrm{~g}, 0.25 \mathrm{mmol})$, polymer-supported nitrite $(0.21 \mathrm{~g}$, $0.75 \mathrm{mmol}), p$-toluenesulfonic acid monohydrate $(0.13 \mathrm{~g}, 0.75$ $\mathrm{mmol})$, palladium(II) acetate $(0.0030,0.013 \mathrm{mmol})$, and methyl acrylate $(0.11 \mathrm{~mL}, 1.3 \mathrm{mmol})$. Purification by neutral alumina flash column chromatography, eluting with $5 \%$ diethyl ether in hexane gave methyl (E)-3-(2'-benzoylphenyl)acrylate (2k) as a yellow oil $(0.047$ g, $71 \%)$. Spectroscopic data were consistent with the literature. ${ }^{27}{ }^{1} \mathrm{H}$ NMR $\left(400 \mathrm{MHz}, \mathrm{CDCl}_{3}\right) \delta 3.72(\mathrm{~s}, 3 \mathrm{H}), 6.38(\mathrm{~d}, J=16.0 \mathrm{~Hz}, 1 \mathrm{H})$, $7.39-7.49(\mathrm{~m}, 4 \mathrm{H}), 7.53(\mathrm{td}, J=7.6,2.4 \mathrm{~Hz}, 1 \mathrm{H}), 7.60(\mathrm{ddt}, J=7.6$, 7.2, $1.2 \mathrm{~Hz}, 1 \mathrm{H}), 7.71-7.82(\mathrm{~m}, 4 \mathrm{H}) ;{ }^{13} \mathrm{C} \mathrm{NMR}\left(101 \mathrm{MHz}, \mathrm{CDCl}_{3}\right)$ $\delta 51.7\left(\mathrm{CH}_{3}\right), 120.5(\mathrm{CH}), 127.3(\mathrm{CH}), 128.6(2 \times \mathrm{CH}), 129.2$ $(\mathrm{CH}), 129.2(\mathrm{CH}), 130.4(2 \times \mathrm{CH}), 130.8(\mathrm{CH}), 133.6(\mathrm{CH}), 133.9$ (C), 137.3 (C), 139.4 (C), $142.0(\mathrm{CH}), 166.8$ (C), 197.2 (C); MS (ESI) $m / z 289\left(\mathrm{M}+\mathrm{Na}^{+}, 100\right)$.

Methyl (E)-3-(2', 4', 6'-Trichlorophenyl)acrylate (2l). The reaction was carried out according to the previously described procedure for methyl (E)-3-(4'-nitrophenyl)acrylate (2a) using 2,4,6-trichloroaniline (11) $(0.050 \mathrm{~g}, 0.25 \mathrm{mmol})$, polymer-supported nitrite $(0.21 \mathrm{~g}$, containing $0.75 \mathrm{mmol}$ of $\mathrm{NO}_{2}$ ), p-toluenesulfonic acid monohydrate $(0.13 \mathrm{~g}, 0.75 \mathrm{mmol})$, palladium(II) acetate $(0.0030 \mathrm{~g}, 0.010 \mathrm{mmol})$, and methyl acrylate $(0.11 \mathrm{~mL}, 1.3 \mathrm{mmol})$. Purification by silica gel flash column chromatography, eluting with $2 \%$ diethyl ether in hexane

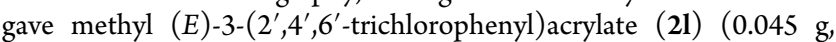
$68 \%$ ) as a pale yellow solid. Mp 64-66 ${ }^{\circ} \mathrm{C}$; IR (neat) 2926, 1726, $1651,1314,1292,1202,1173,970 \mathrm{~cm}^{-1} ;{ }^{1} \mathrm{H}$ NMR (400 MHz, $\left.\mathrm{CDCl}_{3}\right) \delta 3.83(\mathrm{~s}, 3 \mathrm{H}), 6.57(\mathrm{~d}, J=16.4 \mathrm{~Hz}, 1 \mathrm{H}), 7.37(\mathrm{~s}, 2 \mathrm{H}), 7.70$ $(\mathrm{d}, J=16.4 \mathrm{~Hz}, 1 \mathrm{H}) ;{ }^{13} \mathrm{C} \mathrm{NMR}\left(101 \mathrm{MHz}, \mathrm{CDCl}_{3}\right) \delta 52.0\left(\mathrm{CH}_{3}\right)$, $126.8(\mathrm{CH}), 128.8(2 \times \mathrm{CH}), 130.6(\mathrm{C}), 134.9(\mathrm{C}), 135.5(2 \times \mathrm{C})$, $137.3(\mathrm{CH}), 166.4(\mathrm{C})$; MS (ESI) $\mathrm{m} / z 229\left([\mathrm{M}-\mathrm{Cl}]^{+}, 100\right), 207$ (13), 170 (17), 84 (39); HRMS (EI) calcd for $\mathrm{C}_{10} \mathrm{H}_{7}{ }^{35} \mathrm{Cl}_{2} \mathrm{O}_{2}$ ([M $\left.\mathrm{Cl}]^{+}\right)$228.9823, found 228.9821 .

Methyl (E)-3-(2'-Methoxy-5'-methylphenyl)acrylate $(2 m){ }^{28}$ The reaction was carried out according to the previously described procedure for methyl (E)-3-(4'-nitrophenyl)acrylate (2a) using 2- 
methoxy-5-methylaniline $(\mathbf{1 m})(0.050 \mathrm{~g}, 0.36 \mathrm{mmol})$, polymersupported nitrite $\left(0.31 \mathrm{~g}\right.$, containing $1.1 \mathrm{mmol}$ of $\left.\mathrm{NO}_{2}\right)$, $p$ toluenesulfonic acid monohydrate $(0.19 \mathrm{~g}, 1.1 \mathrm{mmol})$, palladium(II) acetate $(0.0040 \mathrm{~g}, 0.018 \mathrm{mmol})$, and methyl acrylate $(0.16 \mathrm{~mL}, 1.8$ $\mathrm{mmol})$. Purification by neutral alumina flash column chromatography, eluting with $10 \%$ diethyl ether in hexane gave methyl $(E)-3-\left(2^{\prime}-\right.$ methoxy-5'-methylphenyl)acrylate (2m) $(0.045 \mathrm{~g}, 60 \%)$ as a yellow oil. Spectroscopic data were consistent with the literature. ${ }^{28}{ }^{1} \mathrm{H}$ NMR $\left(400 \mathrm{MHz}, \mathrm{CDCl}_{3}\right) \delta 2.29(\mathrm{~s}, 3 \mathrm{H}), 3.80(\mathrm{~s}, 3 \mathrm{H}), 3.85(\mathrm{~s}, 3 \mathrm{H}), 6.52$ $(\mathrm{d}, J=16.0 \mathrm{~Hz}, 1 \mathrm{H}), 6.81(\mathrm{~d}, J=8.4 \mathrm{~Hz}, 1 \mathrm{H}), 7.14(\mathrm{dd}, J=8.4,2.0$ $\mathrm{Hz}, 1 \mathrm{H}), 7.31(\mathrm{~d}, J=2.0 \mathrm{~Hz}, 1 \mathrm{H}), 7.97(\mathrm{~d}, J=16.0 \mathrm{~Hz}, 1 \mathrm{H}) ;{ }^{13} \mathrm{C}$ NMR $\left(101 \mathrm{MHz}, \mathrm{CDCl}_{3}\right) \delta 20.4\left(\mathrm{CH}_{3}\right), 51.6\left(\mathrm{CH}_{3}\right), 55.6\left(\mathrm{CH}_{3}\right)$, $111.1(\mathrm{CH}), 118.1(\mathrm{CH}), 123.0(\mathrm{C}), 129.4(\mathrm{CH}), 129.9(\mathrm{C}), 132.0$ (CH), $140.4(\mathrm{CH}), 156.4(\mathrm{C}), 168.0(\mathrm{C})$; MS (ESI) $m / z 229(\mathrm{M}+$ $\left.\mathrm{Na}^{+}, 100\right)$.

(E)-1-Nitro-4-styrylbenzene (2n). ${ }^{29}$ The reaction was carried out according to the previously described procedure for methyl $(E)-3-\left(4^{\prime}\right.$ nitrophenyl)acrylate (2a) using 4-nitroaniline (1a) (0.050 g, 0.36 $\mathrm{mmol})$, polymer-supported nitrite $(0.31 \mathrm{~g}$, containing $1.1 \mathrm{mmol}$ of $\left.\mathrm{NO}_{2}\right), p$-toluenesulfonic acid monohydrate $(0.19 \mathrm{~g}, 1.1 \mathrm{mmol})$, palladium(II) acetate $(0.0080 \mathrm{~g}, 0.036 \mathrm{mmol})$ and styrene $(0.21 \mathrm{~mL}$, $1.8 \mathrm{mmol}$ ). Purification by neutral alumina and then silica gel flash column chromatography, eluting with $10 \%$ diethyl ether in hexane gave (E)-1-nitro-4-styrylbenzene $(\mathbf{2 n})(0.060 \mathrm{~g}, 74 \%)$ as a yellow solid. Mp $154-156{ }^{\circ} \mathrm{C}$ (lit. $\left.{ }^{29} 155-158{ }^{\circ} \mathrm{C}\right) ;{ }^{1} \mathrm{H}$ NMR $(400 \mathrm{MHz}$, $\left.\mathrm{CDCl}_{3}\right) \delta 7.14(\mathrm{~d}, J=16.4 \mathrm{~Hz}, 1 \mathrm{H}), 7.27(\mathrm{~d}, J=16.4 \mathrm{~Hz}, 1 \mathrm{H}), 7.31-$ $7.44(\mathrm{~m}, 3 \mathrm{H}), 7.53-7.58(\mathrm{~m}, 2 \mathrm{H}), 7.63(\mathrm{~d}, J=8.8 \mathrm{~Hz}, 2 \mathrm{H}), 8.22(\mathrm{~d}, J$ $=8.8 \mathrm{~Hz}, 2 \mathrm{H}) ;{ }^{13} \mathrm{C} \mathrm{NMR}\left(101 \mathrm{MHz}, \mathrm{CDCl}_{3}\right) \delta 124.2(2 \times \mathrm{CH})$, $126.3(\mathrm{CH}), 126.9(2 \times \mathrm{CH}), 127.0(2 \times \mathrm{CH}), 128.9(\mathrm{CH}), 128.9(2$ $\times \mathrm{CH}), 133.3(\mathrm{CH}), 136.2(\mathrm{C}), 143.9(\mathrm{C}), 146.8(\mathrm{C}) ; \mathrm{MS}(\mathrm{EI}) \mathrm{m} / \mathrm{z}$ $225\left(\mathrm{M}^{+}, 100\right), 178$ (84), 152 (21), 84 (35).

(E)-4-Fluoro-1-(4"-nitrostyryl)benzene (2o). ${ }^{30}$ The reaction was carried out according to the previously described procedure for methyl (E)-3-(4'-nitrophenyl)acrylate (2a) using 4-nitroaniline (1a) $(0.050 \mathrm{~g}, 0.36 \mathrm{mmol})$, polymer-supported nitrite $(0.31 \mathrm{~g}$, containing $1.1 \mathrm{mmol}$ of $\left.\mathrm{NO}_{2}\right), p$-toluenesulfonic acid monohydrate $(0.17 \mathrm{~g}, 1.1$ $\mathrm{mmol})$, palladium(II) acetate $(0.0040 \mathrm{~g}, 0.018 \mathrm{mmol})$ and 4fluorostyrene $(0.22 \mathrm{~mL}, 1.8 \mathrm{mmol})$. Purification by neutral alumina and then silica gel flash column chromatography, eluting with $10 \%$ diethyl ether in hexane gave (E)-4-fluoro-1-(4"-nitrostyryl)benzene (2o) $(0.040 \mathrm{~g}, 46 \%)$ as a yellow solid. Spectroscopic data were consistent with the literature. ${ }^{30} \mathrm{Mp} 110-113{ }^{\circ} \mathrm{C}$; ${ }^{1} \mathrm{H}$ NMR (400 $\left.\mathrm{MHz}, \mathrm{CDCl}_{3}\right) \delta 7.03-7.13(\mathrm{~m}, 3 \mathrm{H}), 7.22(\mathrm{~d}, J=16.0 \mathrm{~Hz}, 1 \mathrm{H}), 7.49-$ $7.56(\mathrm{~m}, 2 \mathrm{H}), 7.61(\mathrm{~d}, J=8.4 \mathrm{~Hz}, 2 \mathrm{H}), 8.21(\mathrm{~d}, J=8.4 \mathrm{~Hz}, 2 \mathrm{H}) ;{ }^{13} \mathrm{C}$ NMR $\left(101 \mathrm{MHz}, \mathrm{CDCl}_{3}\right) \delta 116.0\left(\mathrm{~d},{ }^{2} J_{\mathrm{C}-\mathrm{F}}=21.9 \mathrm{~Hz}, 2 \times \mathrm{CH}\right)$, $124.2(2 \times \mathrm{CH}), 126.1\left(\mathrm{~d},{ }^{5} J_{\mathrm{C}-\mathrm{F}}=2.4 \mathrm{~Hz}, \mathrm{CH}\right), 126.8(2 \times \mathrm{CH})$, $128.7\left(\mathrm{~d},{ }^{3} J_{\mathrm{C}-\mathrm{F}}=8.1 \mathrm{~Hz}, 2 \times \mathrm{CH}\right), 132.0\left(\mathrm{~d},{ }^{6} J_{\mathrm{C}-\mathrm{F}}=0.8 \mathrm{~Hz}, \mathrm{CH}\right)$, $132.4\left(\mathrm{~d},{ }^{4} J_{\mathrm{C}-\mathrm{F}}=3.3 \mathrm{~Hz}, \mathrm{C}\right), 143.7(\mathrm{C}), 146.8(\mathrm{C}), 163.0\left(\mathrm{~d},{ }^{1} J_{\mathrm{C}-\mathrm{F}}=\right.$ $250.3 \mathrm{~Hz}, \mathrm{C}) ; \mathrm{MS}(\mathrm{EI}) \mathrm{m} / z 243\left(\mathrm{M}^{+}, 72\right), 196$ (62), 179 (32), 84 (100), $51(42)$.

Methyl (2E)-3-(4'-(1'E)-Styrylphenyl)acrylate (3). ${ }^{31}$ To a mixture of methyl (E)-3-(4'-bromophenyl)acrylate (2e) $(0.50 \mathrm{~g}, 0.21 \mathrm{mmol})$, potassium carbonate $(0.058 \mathrm{~g}, 0.42 \mathrm{mmol})$ and bis(triphenylphosphine)palladium(II) dichloride $(0.0070 \mathrm{~g}, 0.011$ $\mathrm{mmol})$ in acetonitrile $(2.5 \mathrm{~mL})$ was added styrene $(0.036 \mathrm{~mL}, 0.31$ $\mathrm{mmol}$ ). The reaction mixture was then heated to $90^{\circ} \mathrm{C}$ and stirred for $24 \mathrm{~h}$. On cooling to room temperature, the mixture was filtered through Celite, washed with diethyl ether $(20 \mathrm{~mL})$ and concentrated in vacuo. Purification by silica gel flash column chromatography, eluting with $5 \%$ diethyl ether in hexane gave methyl $(2 E)-3-\left(4^{\prime}\right.$ $\left(1^{\prime \prime} E\right)$-styrylphenyl)acrylate (3) $(0.040 \mathrm{~g}, 72 \%)$ as a white solid. $\mathrm{Mp}$ $179-181{ }^{\circ} \mathrm{C}$ (lit. $\left.{ }^{31} 183-184{ }^{\circ} \mathrm{C}\right) ;{ }^{1} \mathrm{H}$ NMR (400 MHz, $\left.\mathrm{CDCl}_{3}\right) \delta$ $3.82(\mathrm{~s}, 3 \mathrm{H}), 6.45(\mathrm{~d}, J=16.0 \mathrm{~Hz}, 1 \mathrm{H}), 7.10(\mathrm{~d}, J=16.3 \mathrm{~Hz}, 1 \mathrm{H})$, $7.18(\mathrm{~d}, J=16.3 \mathrm{~Hz}, 1 \mathrm{H}), 7.29(\mathrm{t}, J=7.4 \mathrm{~Hz}, 1 \mathrm{H}), 7.38(\mathrm{t}, J=7.4 \mathrm{~Hz}$ $2 \mathrm{H}), 7.50-7.58(\mathrm{~m}, 6 \mathrm{H}), 7.69(\mathrm{~d}, J=16.0 \mathrm{~Hz}, 1 \mathrm{H}) ;{ }^{13} \mathrm{C}$ NMR $(101$ $\left.\mathrm{MHz}, \mathrm{CDCl}_{3}\right) \delta 51.7\left(\mathrm{CH}_{3}\right), 117.4(\mathrm{CH}), 126.7(2 \times \mathrm{CH}), 126.9(2$ $\times \mathrm{CH}), 127.8(\mathrm{CH}), 128.0(\mathrm{CH}), 128.5(2 \times \mathrm{CH}), 128.8(2 \times \mathrm{CH})$, $130.1(\mathrm{CH}), 133.6(\mathrm{C}), 137.0(\mathrm{C}), 139.4(\mathrm{C}), 144.4(\mathrm{CH}), 167.5$ (C); MS (EI) m/z $264\left(\mathrm{M}^{+}, 100\right), 233$ (10), 203 (12), 189 (6), 178 (8), 83 (22).
Methyl (E)-3-([1', $1^{\prime \prime}-$ Biphenyl]-4'-yl)acrylate $(4 a) .{ }^{32}$ To a mixture of methyl (E)-3-(4'-bromophenyl)acrylate (2e) $(0.50 \mathrm{~g}, 0.21 \mathrm{mmol})$, phenyl boronic acid $(0.26 \mathrm{~g}, 0.21 \mathrm{mmol})$ and $\left[1,1^{\prime}\right.$-bis(diphenylphosphino)ferrocene]palladium(II) dichloride (0.0090 g, $0.011 \mathrm{mmol})$ in dioxane $(4 \mathrm{~mL})$ was added cesium fluoride $(0.064 \mathrm{~g}$, $0.42 \mathrm{mmol}$ ). The reaction mixture was degassed with argon under sonication for $0.5 \mathrm{~h}$ and then heated to $80^{\circ} \mathrm{C}$ for $24 \mathrm{~h}$. On cooling to room temperature, the mixture was filtered through Celite, washed with diethyl ether $(20 \mathrm{~mL})$ and concentrated in vacuo. Purification by silica gel flash column chromatography, eluting with $20 \%$ diethyl ether in hexane gave methyl $(E)-3-\left(\left[1^{\prime}, 1^{\prime \prime}\right.\right.$-biphenyl $]-4^{\prime}$-yl)acrylate (4a) $(0.044 \mathrm{~g}, 88 \%)$ as a pale yellow solid. Mp $146-148^{\circ} \mathrm{C}$ (lit. ${ }^{32} 147-$ $\left.148{ }^{\circ} \mathrm{C}\right) ;{ }^{1} \mathrm{H}$ NMR $\left(400 \mathrm{MHz}, \mathrm{CDCl}_{3}\right) \delta 3.83(\mathrm{~s}, 3 \mathrm{H}), 6.49(\mathrm{~d}, J=$ $16.0 \mathrm{~Hz}, 1 \mathrm{H}), 7.35-7.41(\mathrm{~m}, 1 \mathrm{H}), 7.43-7.50(\mathrm{~m}, 2 \mathrm{H}), 7.57-7.66$ $(\mathrm{m}, 6 \mathrm{H}), 7.75(\mathrm{~d}, J=16.0 \mathrm{~Hz}, 1 \mathrm{H}) ;{ }^{13} \mathrm{C} \mathrm{NMR}\left(101 \mathrm{MHz}, \mathrm{CDCl}_{3}\right) \delta$ $51.7\left(\mathrm{CH}_{3}\right), 117.7(\mathrm{CH}), 127.1(2 \times \mathrm{CH}), 127.6(2 \times \mathrm{CH}), 127.9$ $(\mathrm{CH}), 128.6(2 \times \mathrm{CH}), 128.9(2 \times \mathrm{CH}), 133.4(\mathrm{C}), 140.2(\mathrm{C}), 143.1$ (C), $144.4(\mathrm{CH}), 167.5(\mathrm{C})$; MS (ESI) $m / z 261\left(\mathrm{M}+\mathrm{Na}^{+}, 100\right)$.

Methyl (E)-3-(2'-Methyl-[1', $1^{\prime \prime}$-biphenyl]-4'-yl)acrylate (4b). The reaction was carried out according to the previously described

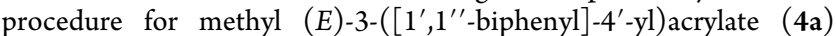
using methyl (E)-3-(4'-bromophenyl)acrylate (2e) (0.50 g, 0.21 mmol), 2-methylphenyl boronic acid (0.44 g, $0.21 \mathrm{mmol})$, [1, $1^{\prime}$ bis(diphenylphosphino)ferrocene $]$ palladium(II) dichloride $(0.0090 \mathrm{~g}$, $0.011 \mathrm{mmol})$ and cesium fluoride $(0.064 \mathrm{~g}, 0.42 \mathrm{mmol})$. Purification by silica gel flash column chromatography, eluting with $20 \%$ diethyl

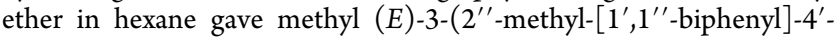
yl)acrylate (4b) $(0.044 \mathrm{~g}, 83 \%)$ as a white solid. Mp 51-53 ${ }^{\circ} \mathrm{C}$; IR (neat) 2949, 1708, 1632, 1313, 1169, 998, 935, $765 \mathrm{~cm}^{-1} ;{ }^{1} \mathrm{H}$ NMR $\left(400 \mathrm{MHz}, \mathrm{CDCl}_{3}\right) \delta 2.27(\mathrm{~s}, 3 \mathrm{H}), 3.81(\mathrm{~s}, 3 \mathrm{H}), 6.47(\mathrm{~d}, J=16.0 \mathrm{~Hz}$, $1 \mathrm{H}), 7.19-7.29(\mathrm{~m}, 4 \mathrm{H}), 7.34(\mathrm{~d}, J=8.8 \mathrm{~Hz}, 2 \mathrm{H}), 7.56(\mathrm{~d}, J=8.8$ $\mathrm{Hz}, 2 \mathrm{H}), 7.74(\mathrm{~d}, J=16.0 \mathrm{~Hz}, 1 \mathrm{H}) ;{ }^{13} \mathrm{C} \mathrm{NMR}\left(101 \mathrm{MHz}, \mathrm{CDCl}_{3}\right) \delta$ $20.4\left(\mathrm{CH}_{3}\right), 51.7\left(\mathrm{CH}_{3}\right), 117.6(\mathrm{CH}), 125.9(\mathrm{CH}), 127.7(\mathrm{CH})$, $127.9(2 \times \mathrm{CH}), 129.6(\mathrm{CH}), 129.8(2 \times \mathrm{CH}), 130.5(\mathrm{CH}), 132.9$ (C), $135.2(\mathrm{C}), 141.0(\mathrm{C}), 144.1(\mathrm{C}), 144.6(\mathrm{CH}), 167.5(\mathrm{C})$; MS (EI) $m / z 252\left(\mathrm{M}^{+}, 100\right), 221(28), 178$ (21), 111 (6), 84 (14); HRMS (EI) calcd for $\mathrm{C}_{17} \mathrm{H}_{16} \mathrm{O}_{2}\left(\mathrm{M}^{+}\right)$252.1150, found 252.1142 .

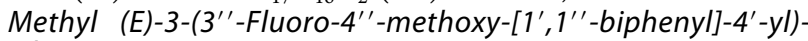
acrylate (4c). The reaction was carried out according to the previously described procedure for methyl $(E)-3-\left(\left[1^{\prime}, 1^{\prime \prime}\right.\right.$-biphenyl $]$ $4^{\prime}$-yl)acrylate (4a) using methyl (E)-3-(4'-bromophenyl)acrylate (2e) $(0.50 \mathrm{~g}, 0.21 \mathrm{mmol})$, 3-fluoro-4-methoxyphenyl boronic acid $(0.36 \mathrm{~g}$, $0.21 \mathrm{mmol}),\left[1,1^{\prime}\right.$-bis(diphenylphosphino)ferrocene $]$ palladium(II) dichloride $(0.0090 \mathrm{~g}, 0.011 \mathrm{mmol})$ and cesium fluoride $(0.064 \mathrm{~g}$, $0.42 \mathrm{mmol}$ ). Purification by silica gel flash column chromatography, eluting with $20 \%$ diethyl ether in hexane gave methyl (E)-3-(3"'-

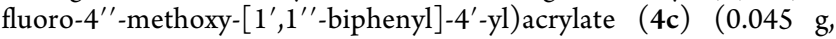
$75 \%$ ) as a white solid. $\mathrm{Mp} 118-121{ }^{\circ} \mathrm{C}$; IR (neat) 2954, 1723, 1316, 1173, 1135, $806 \mathrm{~cm}^{-1}$; ${ }^{1} \mathrm{H} \mathrm{NMR}\left(400 \mathrm{MHz}, \mathrm{CDCl}_{3}\right) \delta 3.82(\mathrm{~s}$, $3 \mathrm{H}), 3.93(\mathrm{~s}, 3 \mathrm{H}), 6.46(\mathrm{~d}, J=16.0 \mathrm{~Hz}, 1 \mathrm{H}), 7.03(\mathrm{t}, J=8.8 \mathrm{~Hz}, 1 \mathrm{H})$, $7.30-7.38(\mathrm{~m}, 2 \mathrm{H}), 7.54(\mathrm{~d}, J=8.8 \mathrm{~Hz}, 2 \mathrm{H}), 7.58(\mathrm{~d}, J=8.8 \mathrm{~Hz}$, $2 \mathrm{H}), 7.71(\mathrm{~d}, J=16.0 \mathrm{~Hz}, 1 \mathrm{H}) ;{ }^{13} \mathrm{C} \mathrm{NMR}\left(101 \mathrm{MHz}, \mathrm{CDCl}_{3}\right) \delta 51.7$ $\left(\mathrm{CH}_{3}\right), 56.4\left(\mathrm{CH}_{3}\right), 113.7\left(\mathrm{~d},{ }^{4} J_{\mathrm{C}-\mathrm{F}}=2.2 \mathrm{~Hz}, \mathrm{CH}\right), 114.7\left(\mathrm{~d},{ }^{2} J_{\mathrm{C}-\mathrm{F}}=\right.$ $19.2 \mathrm{~Hz}, \mathrm{CH}), 117.7(\mathrm{CH}), 122.7\left(\mathrm{~d},{ }^{3} J_{\mathrm{C}-\mathrm{F}}=3.3 \mathrm{~Hz}, \mathrm{CH}\right), 127.0(2 \times$ $\mathrm{CH}), 128.7(2 \times \mathrm{CH}), 133.2(\mathrm{C}), 133.3(\mathrm{C}), 141.5\left(\mathrm{~d},{ }^{3} J_{\mathrm{C}-\mathrm{F}}=1.9\right.$ $\mathrm{Hz}, \mathrm{C}), 144.2(\mathrm{CH}), 147.6\left(\mathrm{~d},{ }^{2} J_{\mathrm{C}-\mathrm{F}}=10.2 \mathrm{~Hz}, \mathrm{C}\right), 152.6\left(\mathrm{~d},{ }^{1} J_{\mathrm{C}-\mathrm{F}}=\right.$ $246.9 \mathrm{~Hz}, \mathrm{C}), 167.4$ (C); MS (ESI) $m / z 309\left(\mathrm{M}+\mathrm{Na}^{+}, 100\right)$; HRMS (ESI) calcd for $\mathrm{C}_{17} \mathrm{H}_{15} \mathrm{FNaO}_{3}\left(\mathrm{M}+\mathrm{Na}^{+}\right)$309.0897, found 309.0899.

Methyl (E)-3-(4'-Allylphenyl)acrylate (4d). The reaction was carried out according to the previously described procedure for methyl (E)-3-([1', $1^{\prime \prime}$-biphenyl $]-4^{\prime}$-yl)acrylate (4a) using methyl $(E)$ 3-(4'-bromophenyl)acrylate (2e) $(0.50 \mathrm{~g}, 0.21 \mathrm{mmol})$, allylboronic acid pinacol ester $(0.060 \mathrm{~mL}, 0.32 \mathrm{mmol}),\left[1,1^{\prime}\right.$-bis(diphenylphosphino)ferrocene $]$ palladium(II) dichloride $(0.0017 \mathrm{~g}$, $0.021 \mathrm{mmol})$, and cesium fluoride $(0.064 \mathrm{~g}, 0.42 \mathrm{mmol})$. Purification by silica gel flash column chromatography, eluting with $20 \%$ diethyl ether in hexane gave methyl (E)-3-(4'-allylphenyl)acrylate (4d) $(0.027 \mathrm{~g}, 64 \%)$ as a yellow oil. IR (neat) $2950,1717,1635,1317$, 1271, 1204, 1166, 983, $826 \mathrm{~cm}^{-1}$; ${ }^{1} \mathrm{H}$ NMR (400 MHz, $\left.\mathrm{CDCl}_{3}\right) \delta$ $3.41(\mathrm{~d}, J=6.8 \mathrm{~Hz}, 2 \mathrm{H}), 3.80(\mathrm{~s}, 3 \mathrm{H}), 5.06-5.13(\mathrm{~m}, 2 \mathrm{H}), 5.95(\mathrm{ddt}$ 
$J=17.2,9.6,6.8 \mathrm{~Hz}, 1 \mathrm{H}), 6.41(\mathrm{~d}, J=16.0 \mathrm{~Hz}, 1 \mathrm{H}), 7.21(\mathrm{~d}, J=8.1$ $\mathrm{Hz}, 2 \mathrm{H}), 7.46(\mathrm{~d}, J=8.1 \mathrm{~Hz}, 2 \mathrm{H}), 7.68(\mathrm{~d}, J=16.0 \mathrm{~Hz}, 1 \mathrm{H}) ;{ }^{13} \mathrm{C}$ NMR $\left(101 \mathrm{MHz}, \mathrm{CDCl}_{3}\right) \delta 40.1\left(\mathrm{CH}_{2}\right), 51.7\left(\mathrm{CH}_{3}\right), 116.4\left(\mathrm{CH}_{2}\right)$, $117.1(\mathrm{CH}), 128.2(2 \times \mathrm{CH}), 129.2(2 \times \mathrm{CH}), 132.4(\mathrm{C}), 136.7$ (CH), $142.8(\mathrm{C}), 144.7(\mathrm{CH}), 167.6(\mathrm{C}) ; \mathrm{MS}(\mathrm{ESI}) \mathrm{m} / z 427$ ([2M + $\left.\mathrm{Na}]^{+}, 100\right)$; HRMS (ESI) calcd for $\mathrm{C}_{26} \mathrm{H}_{28} \mathrm{NaO}_{4}\left([2 \mathrm{M}+\mathrm{Na}]^{+}\right)$ 427.1880, found 427.1886.

Methyl (E)-3-(2'-Nitrophenyl)acrylate $(6 a){ }^{8 d}$ The reaction was carried out according to the previously described procedure for methyl (E)-3-(4'-nitrophenyl)acrylate (2a) using 2-nitroaniline (5a) $(0.055 \mathrm{~g}, 0.40 \mathrm{mmol})$, polymer-supported nitrite $(0.34 \mathrm{~g}$, containing $1.2 \mathrm{mmol}$ of $\left.\mathrm{NO}_{2}\right), p$-toluenesulfonic acid monohydrate $(0.21 \mathrm{~g}, 1.2$ $\mathrm{mmol})$, palladium(II) acetate $(0.0040 \mathrm{~g}, 0.020 \mathrm{mmol})$, and methyl acrylate $(0.18 \mathrm{~mL}, 2.0 \mathrm{mmol})$. Purification by neutral alumina flash column chromatography, eluting with $10 \%$ diethyl ether in hexane gave methyl (E)-3-(2'-nitrophenyl)acrylate $(6 \mathrm{a})(0.070 \mathrm{~g}, 84 \%)$ as a yellow solid. Mp $68-70{ }^{\circ} \mathrm{C}\left(\right.$ lit. $\left.{ }^{8 \mathrm{~d}} 71-72{ }^{\circ} \mathrm{C}\right) ;{ }^{1} \mathrm{H}$ NMR $(400 \mathrm{MHz}$, $\left.\mathrm{CDCl}_{3}\right) \delta 3.83(\mathrm{~s}, 3 \mathrm{H}), 6.37(\mathrm{~d}, J=16.0 \mathrm{~Hz}, 1 \mathrm{H}), 7.51-7.58(\mathrm{~m}$, $1 \mathrm{H}), 7.60-7.69(\mathrm{~m}, 2 \mathrm{H}), 8.04(\mathrm{~d}, J=8.7 \mathrm{~Hz}, 1 \mathrm{H}), 8.12(\mathrm{~d}, J=16.0$ $\mathrm{Hz}, 1 \mathrm{H}) ;{ }^{13} \mathrm{C}$ NMR $\left(101 \mathrm{MHz}, \mathrm{CDCl}_{3}\right) \delta 52.0\left(\mathrm{CH}_{3}\right), 122.9(\mathrm{CH})$, $124.9(\mathrm{CH}), 129.1(\mathrm{CH}), 130.3(\mathrm{CH}), 130.6(\mathrm{C}), 133.5(\mathrm{CH}), 140.2$ (CH), 148.4 (C), 166.2 (C); MS (EI) $m / z 207\left(\mathrm{M}^{+}, 28\right), 175$ (78), 143 (100), 115 (59), 89 (53).

Methyl (E)-3-(4'-Methyl-2'-nitrophenyl)acrylate $(\mathbf{6 b}) .^{8 d}$ The reaction was carried out according to the previously described procedure for methyl (E)-3-(4'-nitrophenyl)acrylate (2a) using 4methyl-2-nitroaniline $(5 \mathbf{b})(0.050 \mathrm{~g}, 0.33 \mathrm{mmol})$, polymer-supported nitrite $\left(0.28 \mathrm{~g}\right.$, containing $0.99 \mathrm{mmol}$ of $\left.\mathrm{NO}_{2}\right), p$-toluenesulfonic acid monohydrate $(0.17 \mathrm{~g}, 0.99 \mathrm{mmol})$, palladium(II) acetate $(0.0040 \mathrm{~g}$, $0.016 \mathrm{mmol})$, and methyl acrylate $(0.15 \mathrm{~mL}, 1.6 \mathrm{mmol})$. Purification by neutral alumina flash column chromatography, eluting with $10 \%$ diethyl ether in hexane gave methyl (E)-3-(4'-methyl-2'-nitrophenyl)acrylate $(6 \mathbf{b})(0.056 \mathrm{~g}, 77 \%)$ as a pale yellow solid. $\mathrm{Mp} 72-74{ }^{\circ} \mathrm{C}$ (lit. $\left.{ }^{8 \mathrm{~d}} 73-75{ }^{\circ} \mathrm{C}\right) ;{ }^{1} \mathrm{H}$ NMR (400 MHz, $\left.\mathrm{CDCl}_{3}\right) \delta 2.45(\mathrm{~s}, 3 \mathrm{H}), 3.81$ $(\mathrm{s}, 3 \mathrm{H}), 6.33(\mathrm{~d}, J=15.6 \mathrm{~Hz}, 1 \mathrm{H}), 7.41-7.46(\mathrm{~m}, 1 \mathrm{H}), 7.52(\mathrm{~d}, J=$ $8.0 \mathrm{~Hz}, 1 \mathrm{H}), 7.82(\mathrm{~d}, J=0.4 \mathrm{~Hz}, 1 \mathrm{H}), 8.06(\mathrm{~d}, J=15.6 \mathrm{~Hz}, 1 \mathrm{H}) ;{ }^{13} \mathrm{C}$ NMR $\left(101 \mathrm{MHz}, \mathrm{CDCl}_{3}\right) \delta 21.1\left(\mathrm{CH}_{3}\right), 52.0\left(\mathrm{CH}_{3}\right), 122.0(\mathrm{CH})$, $125.2(\mathrm{CH}), 127.6(\mathrm{C}), 128.8(\mathrm{CH}), 134.3(\mathrm{CH}), 140.0(\mathrm{CH}), 141.4$ (C), 148.3 (C), 166.4 (C); MS (ESI) $m / z 244\left(\mathrm{M}+\mathrm{Na}^{+}, 100\right)$.

Methyl (E)-3-(5'-Methyl-2'-nitrophenyl)acrylate (6c). The reaction was carried out according to the previously described procedure for methyl (E)-3-(4'-nitrophenyl)acrylate (2a) using 5-methyl-2nitroaniline $(\mathbf{5 c})(0.050 \mathrm{~g}, 0.33 \mathrm{mmol})$, polymer-supported nitrite $\left(0.28 \mathrm{~g}\right.$, containing $0.99 \mathrm{mmol}$ of $\left.\mathrm{NO}_{2}\right), p$-toluenesulfonic acid monohydrate $(0.17 \mathrm{~g}, 0.99 \mathrm{mmol})$, palladium(II) acetate $(0.0060 \mathrm{~g}$, $0.025 \mathrm{mmol})$, and methyl acrylate $(0.15 \mathrm{~mL}, 1.7 \mathrm{mmol})$. Purification by neutral alumina and then silica gel flash column chromatography, eluting with $10 \%$ diethyl ether in hexane gave methyl $(E)-3-\left(5^{\prime}\right.$ methyl-2'-nitrophenyl)acrylate (6c) $(0.055 \mathrm{~g}, 75 \%)$ as a bright yellow solid. Mp $66-70{ }^{\circ} \mathrm{C}$; IR (neat) 2957, 1715, 1634, 1510, 1342, 1260, 976, $824 \mathrm{~cm}^{-1}$; ${ }^{1} \mathrm{H}$ NMR $\left(400 \mathrm{MHz}, \mathrm{CDCl}_{3}\right) \delta 2.46(\mathrm{~s}, 3 \mathrm{H}), 3.82(\mathrm{~s}$, $3 \mathrm{H}), 6.33(\mathrm{~d}, J=15.8 \mathrm{~Hz}, 1 \mathrm{H}), 7.32(\mathrm{dd}, J=8.4,1.2 \mathrm{~Hz}, 1 \mathrm{H}), 7.37-$ $7.42(\mathrm{~m}, 1 \mathrm{H}), 7.97(\mathrm{~d}, J=8.4 \mathrm{~Hz}, 1 \mathrm{H}), 8.14(\mathrm{~d}, J=15.8 \mathrm{~Hz}, 1 \mathrm{H}) ;{ }^{13} \mathrm{C}$ NMR $\left(101 \mathrm{MHz}, \mathrm{CDCl}_{3}\right) \delta 21.5\left(\mathrm{CH}_{3}\right), 52.0\left(\mathrm{CH}_{3}\right), 122.6(\mathrm{CH})$, $125.1(\mathrm{CH}), 129.7(\mathrm{CH}), 130.8(\mathrm{C}), 130.8(\mathrm{CH}), 140.8(\mathrm{CH}), 144.9$ (C), 146.1 (C), 166.3 (C); MS (ESI) $m / z 244\left(\mathrm{M}+\mathrm{Na}^{+}, 100\right)$; HRMS (ESI) calcd for $\mathrm{C}_{11} \mathrm{H}_{11} \mathrm{NNaO}_{4}\left(\mathrm{M}+\mathrm{Na}^{+}\right) 244.0580$, found 244.0577.

Methyl (E)-3-(4',5'-Dimethyl-2'-nitrophenyl)acrylate (6d). The reaction was carried out according to the previously described procedure for methyl (E)-3-(4'-nitrophenyl)acrylate (2a) using 4,5dimethyl-2-nitroaniline $(5 \mathrm{~d})(0.050 \mathrm{~g}, 0.30 \mathrm{mmol})$, polymersupported nitrite $\left(0.26 \mathrm{~g}\right.$, containing $0.90 \mathrm{mmol}$ of $\left.\mathrm{NO}_{2}\right), p$ toluenesulfonic acid monohydrate $(0.16 \mathrm{~g}, 0.90 \mathrm{mmol})$, palladium(II) acetate $(0.0030 \mathrm{~g}, 0.015 \mathrm{mmol})$, and methyl acrylate $(0.14 \mathrm{~mL}, 1.5$ $\mathrm{mmol})$. Purification by neutral alumina flash column chromatography, eluting with $20 \%$ diethyl ether in hexane gave methyl $(E)-3-\left(4^{\prime}, 5^{\prime}-\right.$ dimethyl-2'-nitrophenyl)acrylate $(\mathbf{6 d})(0.050 \mathrm{~g}, 71 \%)$ as an orange solid. Mp $85-87^{\circ} \mathrm{C}$; IR (neat) 2922, 1729, 1514, 1335, 1165, 1024, $839 \mathrm{~cm}^{-1}$; ${ }^{1} \mathrm{H}$ NMR $\left(400 \mathrm{MHz}, \mathrm{CDCl}_{3}\right) \delta 2.35(\mathrm{~s}, 3 \mathrm{H}), 2.36(\mathrm{~s}, 3 \mathrm{H})$, $3.81(\mathrm{~s}, 3 \mathrm{H}), 6.31(\mathrm{~d}, J=16.0 \mathrm{~Hz}, 1 \mathrm{H}), 7.36(\mathrm{~s}, 1 \mathrm{H}), 7.84(\mathrm{~s}, 1 \mathrm{H})$, $8.11(\mathrm{~d}, J=16.0 \mathrm{~Hz}, 1 \mathrm{H}) ;{ }^{13} \mathrm{C}$ NMR $\left(101 \mathrm{MHz}, \mathrm{CDCl}_{3}\right) \delta 19.6$ $\left(\mathrm{CH}_{3}\right), 19.9\left(\mathrm{CH}_{3}\right), 51.9\left(\mathrm{CH}_{3}\right), 121.8(\mathrm{CH}), 125.8(\mathrm{CH}), 128.1$ (C), $129.9(\mathrm{CH}), 139.8$ (C), $140.6(\mathrm{CH}), 143.5$ (C), 146.0 (C), 166.5 (C); MS (ESI) $m / z 258\left(\mathrm{M}+\mathrm{Na}^{+}, 100\right)$; HRMS (ESI) calcd for $\mathrm{C}_{12} \mathrm{H}_{13} \mathrm{NNaO}_{4}\left(\mathrm{M}+\mathrm{Na}^{+}\right) 258.0737$, found 258.0743.

Methyl (E)-3-(4'-Methoxy-2'-nitrophenyl)acrylate (6e). ${ }^{8 d}$ The reaction was carried out according to the previously described procedure for methyl (E)-3-(4'-nitrophenyl)acrylate (2a) using 4methoxy-2-nitroaniline (5e) $(0.050 \mathrm{~g}, 0.30 \mathrm{mmol})$, polymersupported nitrite $\left(0.26 \mathrm{~g}\right.$, containing $0.90 \mathrm{mmol}$ of $\left.\mathrm{NO}_{2}\right), p$ toluenesulfonic acid monohydrate $(0.16 \mathrm{~g}, 0.90 \mathrm{mmol})$, palladium(II) acetate $(0.0030 \mathrm{~g}, 0.015 \mathrm{mmol})$, and methyl acrylate $(0.13 \mathrm{~mL}, 1.5$ $\mathrm{mmol}$ ). Purification by neutral alumina flash column chromatography, eluting with $20 \%$ diethyl ether in hexane gave methyl $(E)-3-\left(4^{\prime}\right.$ methoxy-2'-nitrophenyl)acrylate (6e) $(0.062 \mathrm{~g}, 87 \%)$ as a pale yellow solid. Mp 87-89 ${ }^{\circ} \mathrm{C}\left(\right.$ lit. $\left.{ }^{8 \mathrm{~d}} 90-91{ }^{\circ} \mathrm{C}\right) ;{ }^{1} \mathrm{H}$ NMR $\left(400 \mathrm{MHz}, \mathrm{CDCl}_{3}\right)$ $\delta 3.80(\mathrm{~s}, 3 \mathrm{H}), 3.90(\mathrm{~s}, 3 \mathrm{H}), 6.30(\mathrm{~d}, J=15.8 \mathrm{~Hz}, 1 \mathrm{H}), 7.16(\mathrm{dd}, J=$ $8.7,2.0 \mathrm{~Hz}, 1 \mathrm{H}), 7.49(\mathrm{~d}, J=2.0 \mathrm{~Hz}, 1 \mathrm{H}), 7.57(\mathrm{~d}, J=8.7 \mathrm{~Hz}, 1 \mathrm{H})$, $8.03(\mathrm{~d}, J=15.8 \mathrm{~Hz}, 1 \mathrm{H}) ;{ }^{13} \mathrm{C}$ NMR $\left(101 \mathrm{MHz}, \mathrm{CDCl}_{3}\right) \delta 51.9$ $\left(\mathrm{CH}_{3}\right), 56.1\left(\mathrm{CH}_{3}\right), 109.5(\mathrm{CH}), 120.0(\mathrm{CH}), 121.0(\mathrm{CH}), 122.4$ (C), $130.0(\mathrm{CH}), 139.5(\mathrm{CH}), 149.4(\mathrm{C}), 160.9$ (C), 166.5 (C); MS (ESI) $m / z 260\left(\mathrm{M}+\mathrm{Na}^{+}, 100\right)$.

Methyl (E)-3-(5'-Methoxy-2'-nitrophenyl)acrylate (6f). ${ }^{33}$ The reaction was carried out according to the previously described procedure for methyl (E)-3-(4'-nitrophenyl)acrylate (2a) using 5methoxy-2-nitroaniline $(\mathbf{5 f})(0.050 \mathrm{~g}, 0.30 \mathrm{mmol})$, polymer-supported nitrite $\left(0.26 \mathrm{~g}\right.$, containing $0.90 \mathrm{mmol}$ of $\left.\mathrm{NO}_{2}\right), p$-toluenesulfonic acid monohydrate $(0.16 \mathrm{~g}, 0.90 \mathrm{mmol})$, palladium(II) acetate $(0.0030 \mathrm{~g}$, $0.015 \mathrm{mmol}$ ), and methyl acrylate $(0.13 \mathrm{~mL}, 1.5 \mathrm{mmol})$. Purification by neutral alumina flash column chromatography, eluting with $20 \%$ diethyl ether in hexane gave methyl $(E)-3-\left(5^{\prime}\right.$-methoxy-2' nitrophenyl)acrylate (6f) (0.054 g, 77\%) as an orange solid. Spectroscopic data were consistent with the literature. ${ }^{33} \mathrm{Mp} 98-$ $100{ }^{\circ} \mathrm{C} ;{ }^{1} \mathrm{H}$ NMR $\left(400 \mathrm{MHz}, \mathrm{CDCl}_{3}\right) \delta 3.82(\mathrm{~s}, 3 \mathrm{H}), 3.92(\mathrm{~s}, 3 \mathrm{H})$, $6.29(\mathrm{~d}, J=15.8 \mathrm{~Hz}, 1 \mathrm{H}), 6.95-7.00(\mathrm{~m}, 2 \mathrm{H}), 8.10-8.15(\mathrm{~m}, 1 \mathrm{H})$, $8.20(\mathrm{~d}, J=15.8 \mathrm{~Hz}, 1 \mathrm{H}) ;{ }^{13} \mathrm{C} \mathrm{NMR}\left(101 \mathrm{MHz}, \mathrm{CDCl}_{3}\right) \delta 52.0$ $\left(\mathrm{CH}_{3}\right), 56.1\left(\mathrm{CH}_{3}\right), 114.2(\mathrm{CH}), 114.9(\mathrm{CH}), 122.7(\mathrm{CH}), 127.7$ (CH), 133.8 (C), $141.1(\mathrm{C}), 141.5(\mathrm{CH}), 163.5$ (C), 166.3 (C); MS (ESI) $m / z 260\left(\mathrm{M}+\mathrm{Na}^{+}, 100\right)$.

Methyl (E)-3-(4'-Fluoro-2'-nitrophenyl)acrylate $(6 g){ }^{33}$ The reaction was carried out according to the previously described procedure for methyl (E)-3-(4'-nitrophenyl)acrylate (2a) using 4fluoro-2-nitroaniline $(\mathbf{5 g})(0.05 \mathrm{~g}, 0.32 \mathrm{mmol})$, polymer-supported nitrite $\left(0.27 \mathrm{~g}\right.$, containing $0.96 \mathrm{mmol}$ of $\left.\mathrm{NO}_{2}\right), p$-toluenesulfonic acid monohydrate $(0.17 \mathrm{~g}, 0.96 \mathrm{mmol})$, palladium(II) acetate $(0.0040 \mathrm{~g}$, $0.016 \mathrm{mmol})$, and methyl acrylate $(0.14 \mathrm{~mL}, 1.6 \mathrm{mmol})$. Purification by neutral alumina and then silica gel flash column chromatography, eluting with $10 \%$ diethyl ether in hexane gave methyl $(E)-3-\left(4^{\prime}\right.$ fluoro-2'-nitrophenyl)acrylate $(6 \mathrm{~g})(0.056 \mathrm{~g}, 78 \%)$ as a pale yellow solid. Spectroscopic data were consistent with the literature. ${ }^{33} \mathrm{Mp}$ 87-89 ${ }^{\circ} \mathrm{C} ;{ }^{1} \mathrm{H}$ NMR $\left(400 \mathrm{MHz}, \mathrm{CDCl}_{3}\right) \delta 3.83(\mathrm{~s}, 3 \mathrm{H}), 6.33(\mathrm{~d}, J=$ $16.0 \mathrm{~Hz}, 1 \mathrm{H}), 7.39$ (dddd, $J=8.8,7.2,2.6,0.4 \mathrm{~Hz}, 1 \mathrm{H}), 7.64(\mathrm{dd}, J=$ $8.8,5.9 \mathrm{~Hz}, 1 \mathrm{H}), 7.78(\mathrm{dd}, J=8.0,2.6 \mathrm{~Hz}, 1 \mathrm{H}), 8.06(\mathrm{dd}, J=16.0,0.4$ $\mathrm{Hz}, 1 \mathrm{H}) ;{ }^{13} \mathrm{C} \mathrm{NMR}\left(101 \mathrm{MHz}, \mathrm{CDCl}_{3}\right) \delta 52.1\left(\mathrm{CH}_{3}\right), 112.7\left(\mathrm{~d},{ }^{2} J_{\mathrm{C}-\mathrm{F}}\right.$ $=26.8 \mathrm{~Hz}, \mathrm{CH}), 121.1\left(\mathrm{~d},{ }^{2} J_{\mathrm{C}-\mathrm{F}}=21.5 \mathrm{~Hz}, \mathrm{CH}\right), 123.1\left(\mathrm{~d},{ }^{5} J_{\mathrm{C}-\mathrm{F}}=0.5\right.$ $\mathrm{Hz}, \mathrm{CH}), 126.9\left(\mathrm{~d},{ }^{3} J_{\mathrm{C}-\mathrm{F}}=4.0 \mathrm{~Hz}, \mathrm{C}\right), 130.9\left(\mathrm{~d},{ }^{3} J_{\mathrm{C}-\mathrm{F}}=8.2 \mathrm{~Hz}, \mathrm{CH}\right)$, $139.1(\mathrm{CH}), 148.8(\mathrm{C}), 162.6\left(\mathrm{~d},{ }^{1} J_{\mathrm{C}-\mathrm{F}}=255.1 \mathrm{~Hz}, \mathrm{C}\right), 166.1(\mathrm{C})$; MS (ESI) $m / z 248\left(\mathrm{M}+\mathrm{Na}^{+}, 100\right)$.

Methyl (E)-3-(5'-Chloro-2'-nitrophenyl)acrylate (6h). The reaction was carried out according to the previously described procedure for methyl (E)-3-(4'-nitrophenyl)acrylate (2a) using 5-chloro-2nitroaniline $(5 \mathbf{h})(0.070 \mathrm{~g}, 0.40 \mathrm{mmol})$, polymer-supported nitrite $\left(0.34 \mathrm{~g}\right.$, containing $1.2 \mathrm{mmol}$ of $\left.\mathrm{NO}_{2}\right), p$-toluenesulfonic acid monohydrate $(0.21 \mathrm{~g}, 1.2 \mathrm{mmol})$, palladium(II) acetate $(0.0040 \mathrm{~g}$, $0.020 \mathrm{mmol})$, and methyl acrylate $(0.18 \mathrm{~mL}, 2.0 \mathrm{mmol})$. Purification by neutral alumina and then silica gel flash column chromatography, eluting with $10 \%$ diethyl ether in hexane gave methyl $(E)-3-\left(5^{\prime}\right.$ chloro-2'-nitrophenyl)acrylate $(\mathbf{6 h})(0.066 \mathrm{~g}, 68 \%)$ as a yellow solid. Mp 110-113 ${ }^{\circ} \mathrm{C}$; IR (neat) 2961, 1715, 1516, 1343, 1279, 1250, 
1034, 976, $829 \mathrm{~cm}^{-1}$; ${ }^{1} \mathrm{H}$ NMR (400 MHz, $\mathrm{CDCl}_{3}$ ) $\delta 3.83$ (s, 3H), $6.35(\mathrm{~d}, J=15.8 \mathrm{~Hz}, 1 \mathrm{H}), 7.50(\mathrm{dd}, J=8.8,2.5 \mathrm{~Hz}, 1 \mathrm{H}), 7.59(\mathrm{~d}, J=$ $2.5 \mathrm{~Hz}, 1 \mathrm{H}), 8.03(\mathrm{~d}, J=8.8 \mathrm{~Hz}, 1 \mathrm{H}), 8.08(\mathrm{~d}, J=15.8 \mathrm{~Hz}, 1 \mathrm{H}) ;{ }^{13} \mathrm{C}$ NMR $\left(101 \mathrm{MHz}, \mathrm{CDCl}_{3}\right) \delta 52.2\left(\mathrm{CH}_{3}\right), 124.0(\mathrm{CH}), 126.5(\mathrm{CH})$, $129.2(\mathrm{CH}), 130.2(\mathrm{CH}), 132.5(\mathrm{C}), 139.1(\mathrm{CH}), 140.1(\mathrm{C}), 146.4$ (C), 165.8 (C); MS (ESI) $m / z 264\left(\mathrm{M}+\mathrm{Na}^{+}, 100\right)$; HRMS (ESI) calcd for $\mathrm{C}_{10} \mathrm{H}_{8}{ }^{35} \mathrm{ClNNaO}_{4}\left(\mathrm{M}+\mathrm{Na}^{+}\right)$264.0034, found 264.0036.

Methyl (E)-3-(4'-Bromo-2'-nitrophenyl)acrylate (6i). ${ }^{8 d}$ The reaction was carried out according to the previously described procedure for methyl (E)-3-(4'-nitrophenyl)acrylate (2a) using 4bromo-2-nitroaniline $(\mathbf{5 i})(0.050 \mathrm{~g}, 0.23 \mathrm{mmol})$, polymer-supported nitrite $\left(0.20 \mathrm{~g}\right.$, containing $0.69 \mathrm{mmol}$ of $\left.\mathrm{NO}_{2}\right), p$-toluenesulfonic acid monohydrate $(0.12 \mathrm{~g}, 0.69 \mathrm{mmol})$, palladium(II) acetate $(0.0030 \mathrm{~g}$, $0.012 \mathrm{mmol}$ ), and methyl acrylate $(0.10 \mathrm{~mL}, 1.2 \mathrm{mmol})$. Purification by neutral alumina and then silica gel flash column chromatography, eluting with $10 \%$ diethyl ether in hexane gave methyl (E)-3-(4'bromo-2'-nitrophenyl)acrylate $(6 \mathbf{h})(0.050 \mathrm{~g}, 76 \%)$ as a yellow solid. Mp 84-86 ${ }^{\circ} \mathrm{C}$ (lit. $\left.{ }^{8 \mathrm{~d}} 84-85{ }^{\circ} \mathrm{C}\right) ;{ }^{1} \mathrm{H}$ NMR $\left(400 \mathrm{MHz}, \mathrm{CDCl}_{3}\right) \delta$ $3.83(\mathrm{~s}, 3 \mathrm{H}), 6.37(\mathrm{~d}, J=16.0 \mathrm{~Hz}, 1 \mathrm{H}), 7.51(\mathrm{~d}, J=8.5 \mathrm{~Hz}, 1 \mathrm{H}), 7.77$ $(\mathrm{d}, J=8.5,2.0 \mathrm{~Hz}, 1 \mathrm{H}), 8.03(\mathrm{~d}, J=16.0 \mathrm{~Hz}, 1 \mathrm{H}), 8.18(\mathrm{~d}, J=2.0 \mathrm{~Hz}$, $1 \mathrm{H}) ;{ }^{13} \mathrm{C}$ NMR $\left(101 \mathrm{MHz}, \mathrm{CDCl}_{3}\right) \delta 52.1\left(\mathrm{CH}_{3}\right), 123.4(\mathrm{CH}), 123.8$ (C), $128.0(\mathrm{CH}), 129.4(\mathrm{C}), 130.3(\mathrm{CH}), 136.6(\mathrm{CH}), 139.0(\mathrm{CH})$, 148.5 (C), 166.0 (C); MS (ESI) $m / z 309\left(\mathrm{M}+\mathrm{Na}^{+}, 98\right), 307$ (100).

Methyl (E)-3-(4'-lodo-2'-nitrophenyl)acrylate (6j). The reaction was carried out according to the previously described procedure for methyl (E)-3-(4'-nitrophenyl)acrylate (2a) using 4-iodo-2-nitroaniline $(5 \mathbf{j})(0.050 \mathrm{~g}, 0.19 \mathrm{mmol})$, polymer-supported nitrite $(0.16 \mathrm{~g}$, containing $0.57 \mathrm{mmol}$ of $\mathrm{NO}_{2}$ ), $p$-toluenesulfonic acid monohydrate $(0.098 \mathrm{~g}, 0.57 \mathrm{mmol})$, palladium(II) acetate $(0.0030 \mathrm{~g}, 0.014 \mathrm{mmol})$, and methyl acrylate $(0.085 \mathrm{~mL}, 0.95 \mathrm{mmol})$. Purification by neutral alumina flash column chromatography, eluting with $5 \%$ diethyl ether in hexane gave methyl (E)-3-(4'-iodo-2'-nitrophenyl)acrylate $(\mathbf{6 j})$ $(0.046 \mathrm{~g}, 73 \%)$ as a yellow solid. Mp $83-85{ }^{\circ} \mathrm{C}$; IR (neat) 2951, 1699, 1524, 1356, 1271, 1034, $824 \mathrm{~cm}^{-1}$; ${ }^{1} \mathrm{H}$ NMR $(400 \mathrm{MHz}$, $\left.\mathrm{CDCl}_{3}\right) \delta 3.83(\mathrm{~s}, 3 \mathrm{H}), 6.37(\mathrm{~d}, J=16.0 \mathrm{~Hz}, 1 \mathrm{H}), 7.35(\mathrm{~d}, J=8.2, \mathrm{~Hz}$, $1 \mathrm{H}), 7.97(\mathrm{dd}, J=8.2,0.8 \mathrm{~Hz}, 1 \mathrm{H}), 8.01(\mathrm{~d}, J=16.0 \mathrm{~Hz}, 1 \mathrm{H}), 8.35$ $(\mathrm{d}, J=0.8 \mathrm{~Hz}, 1 \mathrm{H}) ;{ }^{13} \mathrm{C}$ NMR $\left(101 \mathrm{MHz}, \mathrm{CDCl}_{3}\right) \delta 52.1\left(\mathrm{CH}_{3}\right)$, $94.5(\mathrm{C}), 123.4(\mathrm{CH}), 129.9(\mathrm{C}), 130.2(\mathrm{CH}), 133.6(\mathrm{CH}), 139.1$ (CH), $142.5(\mathrm{CH}), 148.4(\mathrm{C}), 166.0(\mathrm{C})$; MS (ESI) $\mathrm{m} / z 356(\mathrm{M}+$ $\left.\mathrm{Na}^{+}, 100\right)$; HRMS (ESI) calcd for $\mathrm{C}_{10} \mathrm{H}_{8} \mathrm{INNaO}_{4}\left(\mathrm{M}+\mathrm{Na}^{+}\right)$ 355.9390, found 355.9391.

Methyl (E)-3-[2'-Nitro-4'-(trifluoromethyl)phenyl]acrylate (6k). ${ }^{34}$ The reaction was carried out according to the previously described procedure for methyl (E)-3-(4'-nitrophenyl)acrylate (2a) using 2nitro-4-(trifluoromethyl)aniline $(5 \mathrm{k})(0.050 \mathrm{~g}, 0.24 \mathrm{mmol})$, polymersupported nitrite $\left(0.21 \mathrm{~g}\right.$, containing $0.72 \mathrm{mmol}$ of $\left.\mathrm{NO}_{2}\right), p$ toluenesulfonic acid monohydrate $(0.12 \mathrm{~g}, 0.72 \mathrm{mmol})$, palladium(II) acetate $(0.0040 \mathrm{~g}, 0.018 \mathrm{mmol})$, and methyl acrylate $(0.11 \mathrm{~mL}, 1.2$ mmol). Purification by neutral alumina and then silica gel flash column chromatography, eluting with $15 \%$ diethyl ether in hexane gave methyl (E)-3-[2'-nitro-4'-(trifluoromethyl)phenyl] acrylate $(6 \mathbf{k})$ $(0.037 \mathrm{~g}, 56 \%)$ as a bright yellow solid. $\mathrm{Mp} 74-76{ }^{\circ} \mathrm{C}$ (lit. ${ }^{34} 77.6-$ $\left.79.3{ }^{\circ} \mathrm{C}\right) ;{ }^{1} \mathrm{H}$ NMR $\left(400 \mathrm{MHz}, \mathrm{CDCl}_{3}\right) \delta 3.84(\mathrm{~s}, 3 \mathrm{H}), 6.42(\mathrm{~d}, J=$ $15.9 \mathrm{~Hz}, 1 \mathrm{H}), 7.78(\mathrm{~d}, J=8.2 \mathrm{~Hz}, 1 \mathrm{H}), 7.90(\mathrm{dd}, J=8.2,1.0 \mathrm{~Hz}, 1 \mathrm{H})$, $8.11(\mathrm{~d}, J=15.9 \mathrm{~Hz}, 1 \mathrm{H}), 8.31(\mathrm{~d}, J=1.0 \mathrm{~Hz}, 1 \mathrm{H}) ;{ }^{13} \mathrm{C} \mathrm{NMR}(101$ $\left.\mathrm{MHz}, \mathrm{CDCl}_{3}\right) \delta 52.2\left(\mathrm{CH}_{3}\right), 122.4\left(\mathrm{q},{ }^{3} J_{\mathrm{C}-\mathrm{F}}=3.9 \mathrm{~Hz}, \mathrm{CH}\right), 122.5(\mathrm{q}$, $\left.{ }^{1} J_{\mathrm{C}-\mathrm{F}}=273.4 \mathrm{~Hz}, \mathrm{C}\right), 124.9(\mathrm{CH}) 130.0\left(\mathrm{q},{ }^{3} J_{\mathrm{C}-\mathrm{F}}=3.4 \mathrm{~Hz}, \mathrm{CH}\right)$, $130.2(\mathrm{CH}), 132.6\left(\mathrm{q},{ }^{2} J_{\mathrm{C}-\mathrm{F}}=35.0 \mathrm{~Hz}, \mathrm{C}\right), 134.1(\mathrm{C}), 138.7(\mathrm{CH})$, 148.1 (C), 165.7 (C); MS (ESI) $m / z 298\left(\mathrm{M}+\mathrm{Na}^{+}, 100\right)$.

4-Phenyl-2-nitroaniline (5I). ${ }^{35}$ To a mixture of 4-iodo-2-nitroaniline $(5 \mathbf{j})(0.20 \mathrm{~g}, 0.75 \mathrm{mmol})$, phenyl boronic acid $(0.10 \mathrm{~g}, 0.83$ $\mathrm{mmol})$ and palladium(II) chloride $(0.0030 \mathrm{~g}, 0.019 \mathrm{mmol})$ in methanol $(3 \mathrm{~mL})$ was added a solution of sodium hydroxide $(0.12 \mathrm{~g}$, $3.0 \mathrm{mmol})$ in water $(1.5 \mathrm{~mL})$ at room temperature. The reaction mixture was degassed with argon under sonication for $0.5 \mathrm{~h}$, stirred for $24 \mathrm{~h}$, and then heated under reflux for a further $2 \mathrm{~h}$. After cooling to room temperature, the solvent was evaporated, and the residue was washed with a $5 \%$ aqueous solution of hydrochloric acid $(5 \mathrm{~mL})$ until the $\mathrm{pH}$ of the solution became neutral. The mixture was extracted with ethyl acetate $(3 \times 15 \mathrm{~mL})$ and the combined organic layers were washed with brine $(2 \times 15 \mathrm{~mL})$. The organic layer was dried $\left(\mathrm{MgSO}_{4}\right)$, filtered, and concentrated in vacuo. Purification by silica gel flash column chromatography, eluting with $20 \%$ diethyl ether in hexane gave 4-phenyl-2-nitroaniline (51) $(0.13 \mathrm{~g}, 83 \%)$ as an orange solid. Mp $166-169{ }^{\circ} \mathrm{C}$ (lit. ${ }^{35} 168-172{ }^{\circ} \mathrm{C}$ ); ${ }^{1} \mathrm{H}$ NMR (400 MHz, $\left.\mathrm{CDCl}_{3}\right) \delta 6.11(\mathrm{br} \mathrm{s}, 2 \mathrm{H}), 6.90(\mathrm{~d}, J=8.6 \mathrm{~Hz}, 1 \mathrm{H}), 7.31-7.37(\mathrm{~m}$, $1 \mathrm{H}), 7.44(\mathrm{t}, J=7.2 \mathrm{~Hz}, 2 \mathrm{H}), 7.53-7.59(\mathrm{~m}, 2 \mathrm{H}), 7.65(\mathrm{dd}, J=8.6$, $2.0 \mathrm{~Hz}, 1 \mathrm{H}), 8.38(\mathrm{~d}, J=2.0 \mathrm{~Hz}, 1 \mathrm{H}) ;{ }^{13} \mathrm{C} \mathrm{NMR}\left(101 \mathrm{MHz}, \mathrm{CDCl}_{3}\right)$ $\delta 119.3(\mathrm{CH}), 123.9(\mathrm{CH}), 126.3(2 \times \mathrm{CH}), 127.4(\mathrm{CH}), 129.0(2 \times$ $\mathrm{CH}), 130.4(\mathrm{C}), 132.5(\mathrm{C}), 134.5(\mathrm{CH}), 138.8(\mathrm{C}), 143.8$ (C); MS (ESI) $m / z 237\left(\mathrm{M}+\mathrm{Na}^{+}, 100\right)$.

Methyl (E)-3-(3'-Nitro-[1', $1^{\prime \prime}$-biphenyl]-4'-yl)acrylate (6I). The reaction was carried out according to the previously described procedure for methyl (E)-3-(4'-nitrophenyl)acrylate (2a) using 4phenyl-2-nitroaniline (51) $(0.026 \mathrm{~g}, 0.12 \mathrm{mmol})$, polymer-supported nitrite $\left(0.10 \mathrm{~g}\right.$, containing $0.36 \mathrm{mmol}$ of $\left.\mathrm{NO}_{2}\right), p$-toluenesulfonic acid monohydrate $(0.062 \mathrm{~g}, 0.36 \mathrm{mmol})$, palladium(II) acetate $(0.0010 \mathrm{~g}$, $0.0060 \mathrm{mmol})$, and methyl acrylate $(0.054 \mathrm{~mL}, 0.60 \mathrm{mmol})$. Purification by neutral alumina flash column chromatography, eluting with $10 \%$ diethyl ether in hexane gave methyl $(E)-3-\left(3^{\prime}\right.$-nitro- $\left[1^{\prime}, 1^{\prime \prime}\right.$ biphenyl] $-4^{\prime}$-yl $)$ acrylate $(61)(0.023 \mathrm{~g}, 68 \%)$ as a yellow solid. Mp 89-91 ${ }^{\circ} \mathrm{C}$; IR (neat) 2920, 1701, 1528, 1350, 1287, 1225, 1032, 760

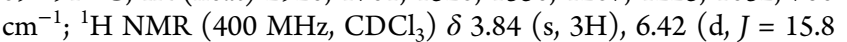
$\mathrm{Hz}, 1 \mathrm{H}), 7.42-7.53(\mathrm{~m}, 3 \mathrm{H}), 7.60-7.65(\mathrm{~m}, 2 \mathrm{H}), 7.71(\mathrm{~d}, J=8.2 \mathrm{~Hz}$, $1 \mathrm{H}), 7.86(\mathrm{ddd}, J=8.2,1.8,0.4 \mathrm{~Hz}, 1 \mathrm{H}), 8.14(\mathrm{~d}, J=15.8 \mathrm{~Hz}, 1 \mathrm{H})$, $8.24(\mathrm{~d}, J=1.8 \mathrm{~Hz}, 1 \mathrm{H}) ;{ }^{13} \mathrm{C}$ NMR $\left(101 \mathrm{MHz}, \mathrm{CDCl}_{3}\right) \delta 52.0$ $\left(\mathrm{CH}_{3}\right), 122.7(\mathrm{CH}), 123.2(\mathrm{CH}), 127.0(2 \times \mathrm{CH}), 128.8(\mathrm{C}), 129.0$ $(\mathrm{CH}), 129.3(2 \times \mathrm{CH}), 129.5(\mathrm{CH}), 131.6(\mathrm{CH}), 137.8(\mathrm{C}), 139.7$ (CH), 143.7 (C), 148.9 (C), 166.3 (C); MS (ESI) $m / z 306(\mathrm{M}+$ $\left.\mathrm{Na}^{+}, 100\right)$; HRMS (ESI) calcd for $\mathrm{C}_{16} \mathrm{H}_{13} \mathrm{NNaO}_{4}\left(\mathrm{M}+\mathrm{Na}^{+}\right)$ 306.0737, found 306.0742 .

4-(4'-Methoxyphenyl)-2-nitroaniline $(5 \mathrm{~m}) .^{36}$ The reaction was carried out according to the previously described procedure for 4phenyl-2-nitroaniline (5l) using 4-iodo-2-nitroaniline $(5 \mathbf{j})$ (0.20 g, $0.75 \mathrm{mmol})$, 4-methoxyphenyl boronic acid $(0.13 \mathrm{~g}, 0.83 \mathrm{mmol})$, palladium(II) chloride $(0.0030 \mathrm{~g}, 0.019 \mathrm{mmol})$ and sodium hydroxide $(0.12 \mathrm{~g}, 3.0 \mathrm{mmol})$. Purification by silica gel flash column chromatography, eluting with $50 \%$ diethyl ether in hexane gave 4(4'-methoxyphenyl)-2-nitroaniline $(\mathbf{5 m})(0.14 \mathrm{~g}, 77 \%)$ as an orange solid. Mp 168-169 ${ }^{\circ} \mathrm{C}$ (lit. $\left.{ }^{36} 170-171{ }^{\circ} \mathrm{C}\right)$; ${ }^{1} \mathrm{H}$ NMR (400 MHz, $\left.\mathrm{CDCl}_{3}\right) \delta 3.85(\mathrm{~s}, 3 \mathrm{H}), 6.07$ (br s, $\left.2 \mathrm{H}\right), 6.87(\mathrm{~d}, J=8.6 \mathrm{~Hz}, 1 \mathrm{H}), 6.96$ $(\mathrm{d}, J=8.7 \mathrm{~Hz}, 2 \mathrm{H}), 7.47(\mathrm{~d}, J=8.7 \mathrm{~Hz}, 2 \mathrm{H}), 7.59(\mathrm{dd}, J=8.6,2.1 \mathrm{~Hz}$, $1 \mathrm{H}), 8.31(\mathrm{dd}, J=2.1 \mathrm{~Hz}, 1 \mathrm{H}) ;{ }^{13} \mathrm{C}$ NMR $\left(101 \mathrm{MHz}, \mathrm{CDCl}_{3}\right) \delta 55.4$ $\left(\mathrm{CH}_{3}\right), 114.4(2 \times \mathrm{CH}), 119.3(\mathrm{CH}), 123.2(\mathrm{CH}), 127.4(2 \times \mathrm{CH})$, 130.2 (C), 131.4 (C), 132.5 (C), 134.3 (CH), 143.4 (C), 159.2 (C); MS (ESI) $m / z 267\left(\mathrm{M}+\mathrm{Na}^{+}, 100\right)$.

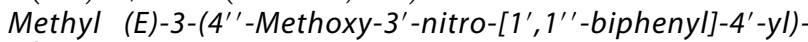
acrylate $(6 \mathrm{~m})$. The reaction was carried out according to the previously described procedure for methyl $(E)-3-\left(4^{\prime}\right.$-nitrophenyl)acrylate (2a) using 4-(4'-methoxyphenyl)-2-nitroaniline (5m) (0.040 g, $0.16 \mathrm{mmol})$, polymer-supported nitrite $(0.14 \mathrm{~g}$, containing 0.48 mmol of $\mathrm{NO}_{2}$ ), $p$-toluenesulfonic acid monohydrate $(0.083 \mathrm{~g}, 0.48$ $\mathrm{mmol})$, palladium(II) acetate $(0.0020 \mathrm{~g}, 0.0080 \mathrm{mmol})$, and methyl acrylate $(0.072 \mathrm{~mL}, 0.80 \mathrm{mmol})$. Purification by neutral alumina and then silica gel flash column chromatography, eluting with $25 \%$ diethyl ether in hexane gave methyl (E)-3- $\left(4^{\prime \prime}\right.$-methoxy-3'-nitro- $\left[1^{\prime}, 1^{\prime \prime}\right.$ biphenyl]-4'-yl)acrylate $(6 \mathrm{~m})(0.038 \mathrm{~g}, 60 \%)$ as a yellow solid. $\mathrm{Mp}$ 90-92 ${ }^{\circ} \mathrm{C}$; IR (neat) 2955, 1690, 1605, 1512, 1350, 1250, 1173, 825 $\mathrm{cm}^{-1}$; ${ }^{1} \mathrm{H}$ NMR $\left(400 \mathrm{MHz} \mathrm{CDCl}_{3}\right) \delta 3.84(\mathrm{~s}, 3 \mathrm{H}), 3.87(\mathrm{~s}, 3 \mathrm{H}), 6.41$ $(\mathrm{d}, J=15.8 \mathrm{~Hz}, 1 \mathrm{H}), 7.01(\mathrm{~d}, J=8.8 \mathrm{~Hz}, 2 \mathrm{H}), 7.57(\mathrm{~d}, J=8.8 \mathrm{~Hz}$, $2 \mathrm{H}), 7.68(\mathrm{~d}, J=8.2 \mathrm{~Hz}, 1 \mathrm{H}), 7.81(\mathrm{dd}, J=8.2,1.8 \mathrm{~Hz}, 1 \mathrm{H}), 8.12(\mathrm{~d}$, $J=15.8 \mathrm{~Hz}, 1 \mathrm{H}), 8.18(\mathrm{~d}, J=1.8 \mathrm{~Hz}, 1 \mathrm{H}) ;{ }^{13} \mathrm{C} \mathrm{NMR}(101 \mathrm{MHz}$, $\left.\mathrm{CDCl}_{3}\right) \delta 52.0\left(\mathrm{CH}_{3}\right), 55.4\left(\mathrm{CH}_{3}\right), 114.7(2 \times \mathrm{CH}), 122.3(\mathrm{CH})$, $122.5(\mathrm{CH}), 128.0(\mathrm{C}), 128.2(2 \times \mathrm{CH}), 129.4(\mathrm{CH}), 130.1(\mathrm{C})$, 130.9 (CH), $139.7(\mathrm{CH}), 143.3(\mathrm{C}), 148.9$ (C), 160.5 (C), 166.3 (C); MS (ESI) $m / z 336\left(\mathrm{M}+\mathrm{Na}^{+}, 100\right)$; HRMS (ESI) calcd for $\mathrm{C}_{17} \mathrm{H}_{15} \mathrm{NNaO}_{5}\left(\mathrm{M}+\mathrm{Na}^{+}\right) 336.0842$, found 336.0833 .

4-(4'-Fluorophenyl)-2-nitroaniline $(5 n))^{37}$ The reaction was carried out according to the previously described procedure for 4phenyl-2-nitroaniline (51) using 4-iodo-2-nitroaniline (5j) (0.20 g, 
$0.75 \mathrm{mmol})$, 4-fluorophenyl boronic acid $(0.12 \mathrm{~g}, 0.83 \mathrm{mmol})$, palladium(II) chloride $(0.0030 \mathrm{~g}, 0.019 \mathrm{mmol})$ and sodium hydroxide $(0.12 \mathrm{~g}, 3.0 \mathrm{mmol})$. Purification by silica gel flash column chromatography, eluting with $50 \%$ diethyl ether in hexane gave 4(4'-fluorophenyl)-2-nitroaniline (5n) $(0.15 \mathrm{~g}, 84 \%)$ as an orange solid. Spectroscopic data were consistent with the literature. ${ }^{37} \mathrm{Mp}$ 136-138 ${ }^{\circ} \mathrm{C}$; ${ }^{1} \mathrm{H}$ NMR (400 MHz, $\mathrm{CDCl}_{3}$ ) $\delta 6.13$ (br s, $2 \mathrm{H}$ ), 6.89 $(\mathrm{d}, J=8.8 \mathrm{~Hz}, 1 \mathrm{H}), 7.12(\mathrm{t}, J=8.8 \mathrm{~Hz}, 2 \mathrm{H}), 7.50(\mathrm{dd}, J=8.8,5.2 \mathrm{~Hz}$ $2 \mathrm{H}), 7.58(\mathrm{dd}, J=8.8,2.2 \mathrm{~Hz}, 1 \mathrm{H}), 8.31(\mathrm{~d}, J=2.2 \mathrm{~Hz}, 1 \mathrm{H}) ;{ }^{13} \mathrm{C}$ NMR $\left(101 \mathrm{MHz}, \mathrm{CDCl}_{3}\right) \delta 115.9\left(\mathrm{~d},{ }^{2} J_{\mathrm{C}-\mathrm{F}}=21.7 \mathrm{~Hz}, 2 \times \mathrm{CH}\right)$, $119.4(\mathrm{CH}), 123.8(\mathrm{CH}), 127.9\left(\mathrm{~d},{ }^{3} J_{\mathrm{C}-\mathrm{F}}=8.1 \mathrm{~Hz}, 2 \times \mathrm{CH}\right), 129.4$ (C), $132.4(\mathrm{C}), 134.4(\mathrm{CH}), 135.0\left(\mathrm{~d},{ }^{4} J_{\mathrm{C}-\mathrm{F}}=3.2 \mathrm{~Hz}, \mathrm{C}\right), 143.8(\mathrm{C})$, $162.4\left(\mathrm{~d},{ }^{1} J_{\mathrm{C}-\mathrm{F}}=247.8 \mathrm{~Hz}, \mathrm{C}\right)$; MS (ESI) $\mathrm{m} / z 255\left(\mathrm{M}+\mathrm{Na}^{+}, 100\right)$.

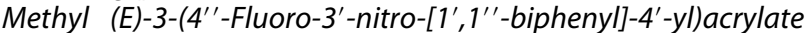
(6n). The reaction was carried out according to the previously described procedure for methyl (E)-3-(4'-nitrophenyl)acrylate (2a) using 4-(4'-fluorophenyl)-2-nitroaniline (5n) $(0.050 \mathrm{~g}, 0.17 \mathrm{mmol})$, polymer-supported nitrite $\left(0.15 \mathrm{~g}\right.$, containing $0.51 \mathrm{mmol}$ of $\left.\mathrm{NO}_{2}\right), p$ toluenesulfonic acid monohydrate $(0.088 \mathrm{~g}, 0.51 \mathrm{mmol})$, palladium(II) acetate $(0.0020 \mathrm{~g}, 0.0090 \mathrm{mmol})$, and methyl acrylate $(0.076 \mathrm{~mL}$, $0.85 \mathrm{mmol}$ ). Purification by neutral alumina flash column chromatography, eluting with $10 \%$ diethyl ether in hexane gave

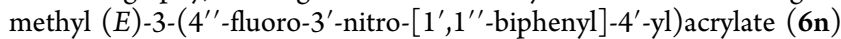
$(0.043 \mathrm{~g}, 84 \%)$ as an orange solid. Mp $124-126^{\circ} \mathrm{C}$; IR (neat) 2922, 1730, 1514, 1435, 1350, 1165, 1030, $839 \mathrm{~cm}^{-1}$; ${ }^{1} \mathrm{H}$ NMR (400 MHz, $\left.\mathrm{CDCl}_{3}\right) \delta 3.84(\mathrm{~s}, 3 \mathrm{H}), 6.42(\mathrm{~d}, J=15.6 \mathrm{~Hz}, 1 \mathrm{H}), 7.19(\mathrm{t}, J=8.8 \mathrm{~Hz}$, $2 \mathrm{H}), 7.60(\mathrm{dd}, J=8.8,5.2 \mathrm{~Hz}, 2 \mathrm{H}), 7.71(\mathrm{~d}, J=8.0 \mathrm{~Hz}, 1 \mathrm{H}), 7.82$ $(\mathrm{dd}, J=8.0,1.6 \mathrm{~Hz}, 1 \mathrm{H}), 8.13(\mathrm{~d}, J=15.6 \mathrm{~Hz}, 1 \mathrm{H}), 8.20(\mathrm{~d}, J=1.6$ $\mathrm{Hz}, 1 \mathrm{H}) ;{ }^{13} \mathrm{C}$ NMR $\left(101 \mathrm{MHz}, \mathrm{CDCl}_{3}\right) \delta 52.0\left(\mathrm{CH}_{3}\right), 116.3\left(\mathrm{~d},{ }^{2} J_{\mathrm{C}-\mathrm{F}}\right.$ $=22.9 \mathrm{~Hz}, 2 \times \mathrm{CH}), 122.8(\mathrm{CH}), 123.0(\mathrm{CH}), 128.8\left(\mathrm{~d},{ }^{3} J_{\mathrm{C}-\mathrm{F}}=9.7\right.$ $\mathrm{Hz}, 2 \times \mathrm{CH}), 128.9(\mathrm{C}), 129.6(\mathrm{CH}), 131.4(\mathrm{CH}), 134.0\left(\mathrm{~d},{ }^{4} J_{\mathrm{C}-\mathrm{F}}=\right.$ $3.2 \mathrm{~Hz}, \mathrm{C}), 139.5(\mathrm{CH}), 142.7(\mathrm{C}), 148.9(\mathrm{C}), 163.4\left(\mathrm{~d},{ }^{1} J_{\mathrm{C}-\mathrm{F}}=\right.$ $250.5 \mathrm{~Hz}, \mathrm{C}), 166.2$ (C); MS (EI) $\mathrm{m} / z 301\left(\mathrm{M}^{+}, 8\right), 255$ (15), 214 (19), 183 (12), 105 (7), 84 (100); HRMS (EI) calcd for $\mathrm{C}_{16} \mathrm{H}_{12} \mathrm{FNO}_{4}\left(\mathrm{M}^{+}\right)$301.0750, found 301.0759.

3,4-Dihydro-1H-quinolin-2-one $(7 a) .{ }^{86}$ The reaction was carried out according to the previously described procedure for methyl $(E)-3$ ( $2^{\prime}$-nitrophenyl)acrylate (2a) using 2-nitroaniline (5a) (0.055 g, 0.40 $\mathrm{mmol})$, except that after the first two steps, the mixture was purged with hydrogen gas and then hydrogenated at atmospheric pressure for a further $24 \mathrm{~h}$ at $40{ }^{\circ} \mathrm{C}$. The reaction mixture was cooled to room temperature, filtered through a short pad of Celite, washed with methanol $(20 \mathrm{~mL})$, and concentrated in vacuo. Purification by silica gel flash column chromatography, eluting with $20 \%$ ethyl acetate in hexane gave 3,4-dihydro- $1 \mathrm{H}$-quinolin-2-one (7a) $(0.046 \mathrm{~g}, 73 \%)$ as a white solid. Mp $162-164{ }^{\circ} \mathrm{C}$ (lit. $\left.{ }^{8 b} 164{ }^{\circ} \mathrm{C}\right) ;{ }^{1} \mathrm{H}$ NMR $(400 \mathrm{MHz}$, $\left.\mathrm{CDCl}_{3}\right) \delta 2.65(\mathrm{dd}, J=8.8,8.0 \mathrm{~Hz}, 2 \mathrm{H}), 2.97(\mathrm{dd}, J=8.0,7.2 \mathrm{~Hz}$, $2 \mathrm{H}), 6.82(\mathrm{~d}, J=7.6 \mathrm{~Hz}, 1 \mathrm{H}), 6.99(\mathrm{td}, J=7.6,1.1 \mathrm{~Hz}, 1 \mathrm{H}), 7.13-$ $7.20(\mathrm{~m}, 2 \mathrm{H}), 8.87(\mathrm{br} \mathrm{s}, 1 \mathrm{H}) ;{ }^{13} \mathrm{C}$ NMR $\left(101 \mathrm{MHz}, \mathrm{CDCl}_{3}\right) \delta 25.4$ $\left(\mathrm{CH}_{2}\right), 30.7\left(\mathrm{CH}_{2}\right), 115.5(\mathrm{CH}), 123.1(\mathrm{CH}), 123.7(\mathrm{C}), 127.5$ (CH), $127.9(\mathrm{CH}), 137.3(\mathrm{C}), 172.0(\mathrm{C}) ; \mathrm{MS}(\mathrm{ESI}) \mathrm{m} / z 170(\mathrm{M}+$ $\left.\mathrm{Na}^{+}, 100\right)$

7-Methyl-3,4-dihydro-1H-quinolin-2-one (7b). ${ }^{38}$ The reaction was carried out according to the previously described procedure for 3,4-dihydro-1H-quinolin-2-one (7a) using 4-methyl-2-nitroaniline (5b) $(0.050 \mathrm{~g}, 0.33 \mathrm{mmol})$, polymer-supported nitrite $(0.28 \mathrm{~g}$, containing $0.99 \mathrm{mmol}$ of $\mathrm{NO}_{2}$ ), p-toluenesulfonic acid monohydrate $(0.17 \mathrm{~g}, 0.99 \mathrm{mmol})$, palladium(II) acetate $(0.0040 \mathrm{~g}, 0.016 \mathrm{mmol})$, and methyl acrylate $(0.15 \mathrm{~mL}, 1.6 \mathrm{mmol})$. Purification by silica gel flash column chromatography, eluting with $40 \%$ ethyl acetate in hexane gave 7-methyl-3,4-dihydro- $1 \mathrm{H}$-quinolin-2-one (7b) (0.042 g, $79 \%)$ as a pale yellow solid. Mp $133-135{ }^{\circ} \mathrm{C}$ (lit. $\left.{ }^{38} 135-137^{\circ} \mathrm{C}\right) ;{ }^{1} \mathrm{H}$ NMR $\left(400 \mathrm{MHz}, \mathrm{CDCl}_{3}\right) \delta 2.30(\mathrm{~s}, 3 \mathrm{H}), 2.63(\mathrm{dd}, J=8.4,7.6 \mathrm{~Hz}$, $2 \mathrm{H}), 2.92(\mathrm{dd}, J=7.6,7.2 \mathrm{~Hz}, 2 \mathrm{H}), 6.68(\mathrm{~s}, 1 \mathrm{H}), 6.79(\mathrm{~d}, J=7.4 \mathrm{~Hz}$, $1 \mathrm{H}), 7.03(\mathrm{~d}, J=7.4 \mathrm{~Hz}, 1 \mathrm{H}), 9.41$ (br s, $1 \mathrm{H}) ;{ }^{13} \mathrm{C} \mathrm{NMR}(101 \mathrm{MHz}$, $\left.\mathrm{CDCl}_{3}\right) \delta 21.1\left(\mathrm{CH}_{3}\right), 25.0\left(\mathrm{CH}_{2}\right), 30.9\left(\mathrm{CH}_{2}\right), 116.2(\mathrm{CH}), 120.6$ (C), $123.8(\mathrm{CH}), 127.7(\mathrm{CH}), 137.2(\mathrm{C}), 137.5(\mathrm{C}), 172.6(\mathrm{C}) ; \mathrm{MS}$ (EI) $\mathrm{m} / z 161\left(\mathrm{M}^{+}, 100\right), 133$ (55), 106 (20), 86 (46), 84 (59).

6-Methyl-3,4-dihydro-1H-quinolin-2-one (7c). ${ }^{39}$ The reaction was carried out according to the previously described procedure for 3,4-
dihydro-1H-quinolin-2-one (7a) using 5-methyl-2-nitroaniline (5c) $(0.050 \mathrm{~g}, 0.33 \mathrm{mmol})$, polymer-supported nitrite $(0.28 \mathrm{~g}$, containing $0.99 \mathrm{mmol}$ of $\left.\mathrm{NO}_{2}\right)$, $p$-toluenesulfonic acid monohydrate $(0.17 \mathrm{~g}, 0.99$ $\mathrm{mmol})$, palladium(II) acetate $(0.0040 \mathrm{~g}, 0.016 \mathrm{mmol})$, and methyl acrylate $(0.15 \mathrm{~mL}, 1.6 \mathrm{mmol})$. Purification by silica gel flash column chromatography, eluting with $40 \%$ ethyl acetate in hexane gave 6methyl-3,4-dihydro- $1 \mathrm{H}$-quinolin-2-one $(7 \mathrm{c})(0.034 \mathrm{~g}, 64 \%)$ as a white solid. Spectroscopic data were consistent with the literature. ${ }^{39} \mathrm{Mp}$ 120-123 ${ }^{\circ} \mathrm{C}$; ${ }^{1} \mathrm{H}$ NMR $\left(400 \mathrm{MHz}, \mathrm{CDCl}_{3}\right) \delta 2.29(\mathrm{~s}, 3 \mathrm{H}), 2.62$ (dd, $J=7.6,6.0 \mathrm{~Hz}, 2 \mathrm{H}), 2.92(\mathrm{t}, J=7.6 \mathrm{~Hz}, 2 \mathrm{H}), 6.75(\mathrm{~d}, J=8.8 \mathrm{~Hz}, 1 \mathrm{H})$, 6.93-7.00 (m, 2H), 9.26 (br s, $1 \mathrm{H}) ;{ }^{13} \mathrm{C}$ NMR $\left(101 \mathrm{MHz}, \mathrm{CDCl}_{3}\right) \delta$ $20.8\left(\mathrm{CH}_{3}\right), 25.4\left(\mathrm{CH}_{2}\right), 30.8\left(\mathrm{CH}_{2}\right), 115.5(\mathrm{CH}), 123.5(\mathrm{C}), 127.9$ (CH), $128.5(\mathrm{CH}), 132.6$ (C), 134.9 (C), 172.2 (C); MS (EI) $\mathrm{m} / z$ $161\left(\mathrm{M}^{+}, 100\right), 132$ (62), 106 (23), $91(20)$.

6,7-Dimethyl-3,4-dihydro-1H-quinolin-2-one $(7 d) .{ }^{86}$ The reaction was carried out according to the previously described procedure for 3,4-dihydro- $1 \mathrm{H}$-quinolin-2-one (7a) using 4,5-dimethyl-2-nitroaniline (5d) $(0.050 \mathrm{~g}, 0.32 \mathrm{mmol})$, polymer-supported nitrite $(0.27 \mathrm{~g}$, containing $0.96 \mathrm{mmol}$ of $\mathrm{NO}_{2}$ ), $p$-toluenesulfonic acid monohydrate $(0.17 \mathrm{~g}, 0.96 \mathrm{mmol})$, palladium(II) acetate $(0.0040 \mathrm{~g}, 0.016 \mathrm{mmol})$, and methyl acrylate $(0.14 \mathrm{~mL}, 1.6 \mathrm{mmol})$. Purification by silica gel flash column chromatography, eluting with $10 \%$ ethyl acetate in hexane gave 6,7-dimethyl-3,4-dihydro- $1 \mathrm{H}$-quinolin-2-one (7d) $(0.041$ g, $77 \%$ ) as a pale yellow solid. Mp $196-198{ }^{\circ} \mathrm{C}$ (lit. $\left.{ }^{8 \mathrm{~b}} 200{ }^{\circ} \mathrm{C}\right) ;{ }^{1} \mathrm{H}$ $\operatorname{NMR}\left(400 \mathrm{MHz}, \mathrm{CDCl}_{3}\right) \delta 2.20(\mathrm{~s}, 3 \mathrm{H}), 2.21(\mathrm{~s}, 3 \mathrm{H}), 2.61(\mathrm{dd}, J=$ 9.2, 8.0 Hz, 2H), $2.90(\mathrm{dd}, J=8.0,7.2 \mathrm{~Hz}, 2 \mathrm{H}), 6.61(\mathrm{~s}, 1 \mathrm{H}), 6.92(\mathrm{~s}$, $1 \mathrm{H}), 8.76($ br s, $1 \mathrm{H}) ;{ }^{13} \mathrm{C} \mathrm{NMR}\left(101 \mathrm{MHz}, \mathrm{CDCl}_{3}\right) \delta 19.0\left(\mathrm{CH}_{3}\right)$, $19.4\left(\mathrm{CH}_{3}\right), 24.9\left(\mathrm{CH}_{2}\right), 31.0\left(\mathrm{CH}_{2}\right), 116.7(\mathrm{CH}), 120.8(\mathrm{C}), 129.0$ (CH), 131.1 (C), 135.0 (C), 135.8 (C), 172.1 (C); MS (EI) $m / z 175$ $\left(\mathrm{M}^{+}, 100\right), 147$ (29), 133 (16), 83 (21).

7-Methoxy-3,4-dihydro-1H-quinolin-2-one (7e). ${ }^{8 \mathrm{C}}$ The reaction was carried out according to the previously described procedure for 3,4-dihydro-1H-quinolin-2-one (7a) using 4-methoxy-2-nitroaniline (5e) $(0.40 \mathrm{~g}, 2.4 \mathrm{mmol})$, polymer-supported nitrite $(2.0 \mathrm{~g}$, containing $7.1 \mathrm{mmol}$ of $\left.\mathrm{NO}_{2}\right), p$-toluenesulfonic acid monohydrate $(1.2 \mathrm{~g}, 7.1$ mmol), palladium(II) acetate $(0.027 \mathrm{~g}, 0.12 \mathrm{mmol})$, and methyl acrylate $(1.1 \mathrm{~mL}, 12 \mathrm{mmol})$. After the first two steps were complete $(2$ $\mathrm{h})$, the vessel was transferred to a Parr Shaker Hydrogenation apparatus and hydrogenated at $2.5 \mathrm{bar}$ and $\mathrm{rt}$, for $24 \mathrm{~h}$. Purification by silica gel flash column chromatography, eluting with $50 \%$ ethyl acetate in hexane gave 7-methoxy-3,4-dihydro- $1 \mathrm{H}$-quinolin-2-one (7e) $(0.31$ g, 74\%) as a white solid. Mp $141-144{ }^{\circ} \mathrm{C}$ (lit. $\left.{ }^{8 \mathrm{c}} 145{ }^{\circ} \mathrm{C}\right) ;{ }^{1} \mathrm{H}$ NMR $\left(400 \mathrm{MHz} \mathrm{CDCl}_{3}\right) \delta 2.62(\mathrm{dd}, J=7.6,6.0 \mathrm{~Hz}, 2 \mathrm{H}), 2.86(\mathrm{t}, J=7.6$ $\mathrm{Hz}, 2 \mathrm{H}), 3.77(\mathrm{~s}, 3 \mathrm{H}), 6.41(\mathrm{~d}, J=2.5 \mathrm{~Hz}, 1 \mathrm{H}), 6.52(\mathrm{dd}, J=8.3,2.5$ $\mathrm{Hz}, 1 \mathrm{H}), 7.04(\mathrm{~d}, J=8.3 \mathrm{~Hz}, 1 \mathrm{H}), 9.24($ br s, $1 \mathrm{H}) ;{ }^{13} \mathrm{C} \mathrm{NMR}(101$ $\left.\mathrm{MHz}, \mathrm{CDCl}_{3}\right) \delta 24.6\left(\mathrm{CH}_{2}\right), 31.1\left(\mathrm{CH}_{2}\right), 55.5\left(\mathrm{CH}_{3}\right), 101.7(\mathrm{CH})$, $108.3(\mathrm{CH}), 115.8(\mathrm{C}), 128.6(\mathrm{CH}), 138.3(\mathrm{C}), 159.3(\mathrm{C}), 172.5$ (C); MS (ESI) $m / z 200\left(\mathrm{M}+\mathrm{Na}^{+}, 100\right)$.

6-Methoxy-3,4-dihydro-1H-quinolin-2-one (7f). ${ }^{16}$ The reaction was carried out according to the previously described procedure for 7 methoxy-3,4-dihydro-1H-quinolin-2-one (7a) using 5-methoxy-2nitroaniline $(\mathbf{5 f})(0.050 \mathrm{~g}, 0.30 \mathrm{mmol})$, polymer-supported nitrite $\left(0.26 \mathrm{~g}\right.$, containing $0.90 \mathrm{mmol}$ of $\left.\mathrm{NO}_{2}\right), p$-toluenesulfonic acid monohydrate $(0.16 \mathrm{~g}, 0.90 \mathrm{mmol})$, palladium(II) acetate $(0.0030 \mathrm{~g}$, $0.015 \mathrm{mmol})$, and methyl acrylate $(0.13 \mathrm{~mL}, 1.5 \mathrm{mmol})$. Purification by silica gel flash column chromatography, eluting with $50 \%$ ethyl acetate in hexane gave 6-methoxy-3,4-dihydro- $1 \mathrm{H}$-quinolin-2-one (7f) $(0.030 \mathrm{~g}, 57 \%)$ as a white solid. $\mathrm{Mp} 140-142{ }^{\circ} \mathrm{C}\left(\right.$ lit. $\left.^{16} 146-148{ }^{\circ} \mathrm{C}\right)$; ${ }^{1} \mathrm{H}$ NMR $\left(400 \mathrm{MHz}, \mathrm{CDCl}_{3}\right) \delta 2.61(\mathrm{dd}, J=8.0,7.2 \mathrm{~Hz}, 2 \mathrm{H}), 2.93$ (dd, $J=8.0,7.2 \mathrm{~Hz}, 2 \mathrm{H}), 3.77(\mathrm{~s}, 3 \mathrm{H}), 6.69(\mathrm{~d}, J=2.4 \mathrm{~Hz}, 1 \mathrm{H}), 6.71$ (d, $J=7.6 \mathrm{~Hz}, 1 \mathrm{H}), 6.77(\mathrm{dd}, J=7.6,2.4 \mathrm{~Hz}, 1 \mathrm{H}), 9.11(\mathrm{br} \mathrm{s}, 1 \mathrm{H})$; ${ }^{13} \mathrm{C}$ NMR $\left(101 \mathrm{MHz}, \mathrm{CDCl}_{3}\right) \delta 25.7\left(\mathrm{CH}_{2}\right), 30.6\left(\mathrm{CH}_{2}\right), 55.6$ $\left(\mathrm{CH}_{3}\right), 112.4(\mathrm{CH}), 113.8(\mathrm{CH}), 116.3(\mathrm{CH}), 125.0(\mathrm{C}), 130.9(\mathrm{C})$, 155.6 (C), 171.8 (C); MS (ESI) $m / z 200\left(\mathrm{M}+\mathrm{Na}^{+}, 100\right)$.

7-Fluoro-3,4-dihydro-1H-quinolin-2-one $(\mathbf{7 g}) .{ }^{17}$ The reaction was carried out according to the previously described procedure for 3,4dihydro- $1 \mathrm{H}$-quinolin-2-one (7a) using 4-fluoro-2-nitroaniline $(\mathbf{5 g})$ $(0.050 \mathrm{~g}, 0.32 \mathrm{mmol})$, polymer-supported nitrite $(0.27 \mathrm{~g}$, containing $0.96 \mathrm{mmol}$ of $\left.\mathrm{NO}_{2}\right)$, $p$-toluenesulfonic acid monohydrate $(0.17 \mathrm{~g}, 0.96$ mmol), palladium(II) acetate $(0.0040 \mathrm{~g}, 0.016 \mathrm{mmol})$, and methyl 
acrylate $(0.14 \mathrm{~mL}, 1.6 \mathrm{mmol})$. Purification by silica gel flash column chromatography, eluting with $10 \%$ ethyl acetate in hexane gave 7 fluoro-3,4-dihydro-1H-quinolin-2-one $(7 \mathrm{~g})(0.037 \mathrm{~g}, 70 \%)$ as a pale yellow solid. Spectroscopic data were consistent with the literature. ${ }^{17}$ Mp 176-178 ${ }^{\circ} \mathrm{C}$; ${ }^{1} \mathrm{H}$ NMR $\left(400 \mathrm{MHz}, \mathrm{CDCl}_{3}\right) \delta 2.64$ (dd, $J=8.8$, $8.0 \mathrm{~Hz}, 2 \mathrm{H}), 2.94(\mathrm{dd}, J=8.0,7.2 \mathrm{~Hz}, 2 \mathrm{H}), 6.59(\mathrm{dd}, J=9.2,2.4 \mathrm{~Hz}$, $1 \mathrm{H}), 6.68(\mathrm{td}, J=8.2,2.4 \mathrm{~Hz}, 1 \mathrm{H}), 7.09(\mathrm{dd}, J=8.2,6.0 \mathrm{~Hz}, 1 \mathrm{H})$, $9.23($ br s, $1 \mathrm{H}) ;{ }^{13} \mathrm{C}$ NMR $\left(101 \mathrm{MHz}, \mathrm{CDCl}_{3}\right) \delta 24.7\left(\mathrm{CH}_{2}\right), 30.7$ $\left(\mathrm{CH}_{2}\right), 103.1\left(\mathrm{~d},{ }^{2} J_{\mathrm{C}-\mathrm{F}}=25.7 \mathrm{~Hz}, \mathrm{CH}\right), 109.5\left(\mathrm{~d},{ }^{2} J_{\mathrm{C}-\mathrm{F}}=21.4 \mathrm{~Hz}\right.$, $\mathrm{CH}), 119.2\left(\mathrm{~d},{ }^{4} J_{\mathrm{C}-\mathrm{F}}=3.2 \mathrm{~Hz}, \mathrm{C}\right), 129.0\left(\mathrm{~d},{ }^{3} J_{\mathrm{C}-\mathrm{F}}=9.3 \mathrm{~Hz}, \mathrm{CH}\right)$, $138.6\left(\mathrm{~d},{ }^{3} J_{\mathrm{C}-\mathrm{F}}=10.6 \mathrm{~Hz}, \mathrm{C}\right), 162.1\left(\mathrm{~d},{ }^{1} J_{\mathrm{C}-\mathrm{F}}=245.3 \mathrm{~Hz}, \mathrm{C}\right), 172.2$ (C); MS (ESI) $m / z 188\left(\mathrm{M}+\mathrm{Na}^{+}, 100\right)$.

7-Phenyl-3,4-dihydro-1H-quinolin-2-one (7h). The reaction was carried out according to the previously described procedure for 7 methoxy-3,4-dihydro-1H-quinolin-2-one (7e) using 4-phenyl-2-nitroaniline $(51)(0.050 \mathrm{~g}, 0.32 \mathrm{mmol})$, polymer-supported nitrite $(0.27 \mathrm{~g}$, containing $0.96 \mathrm{mmol}$ of $\mathrm{NO}_{2}$ ), $p$-toluenesulfonic acid monohydrate $(0.17 \mathrm{~g}, 0.96 \mathrm{mmol})$, palladium(II) acetate $(0.0040 \mathrm{~g}, 0.016 \mathrm{mmol})$, and methyl acrylate $(0.14 \mathrm{~mL}, 1.6 \mathrm{mmol})$. Purification by silica gel flash column chromatography, eluting with $60 \%$ ethyl acetate in hexane gave 7-phenyl-3,4-dihydro- $1 \mathrm{H}$-quinolin-2-one $(7 \mathbf{h})(0.030 \mathrm{~g}$, $71 \%)$ as a white solid. Mp $178-181{ }^{\circ} \mathrm{C}$; IR (neat) $2976,1674,1483$, 1391, 820, $760 \mathrm{~cm}^{-1} ;{ }^{1} \mathrm{H}$ NMR $\left(400 \mathrm{MHz}, \mathrm{CDCl}_{3}\right) \delta 2.68(\mathrm{t}, J=7.6$ $\mathrm{Hz}, 2 \mathrm{H}), 3.01(\mathrm{t}, J=7.6 \mathrm{~Hz}, 2 \mathrm{H}), 7.02(\mathrm{~s}, 1 \mathrm{H}), 7.19-7.25(\mathrm{~m}, 2 \mathrm{H})$, $7.35(\mathrm{t}, J=7.6 \mathrm{~Hz}, 1 \mathrm{H}), 7.43(\mathrm{t}, J=7.6 \mathrm{~Hz}, 2 \mathrm{H}), 7.56(\mathrm{~d}, J=7.6 \mathrm{~Hz}$, $2 \mathrm{H}), 8.86($ br s, $1 \mathrm{H}) ;{ }^{13} \mathrm{C} \mathrm{NMR}\left(101 \mathrm{MHz}, \mathrm{CDCl}_{3}\right) \delta 25.1\left(\mathrm{CH}_{2}\right)$, $30.8\left(\mathrm{CH}_{2}\right), 114.1(\mathrm{CH}), 121.9(\mathrm{CH}), 122.7(\mathrm{C}), 127.1(2 \times \mathrm{CH})$, $127.5(\mathrm{CH}), 128.3(\mathrm{CH}), 128.8(2 \times \mathrm{CH}), 137.8(\mathrm{C}), 140.4(\mathrm{C})$, 141.0 (C), 172.0 (C); MS (EI) $m / z 223\left(\mathrm{M}^{+}, 100\right), 195$ (38), 168 (14), 152 (10), 83 (51), 75 (30); HRMS (EI) calcd for $\mathrm{C}_{15} \mathrm{H}_{13} \mathrm{NO}$ $\left(\mathrm{M}^{+}\right)$223.0997, found 223.0989.

7-(4'-Methoxyphenyl)-3,4-dihydro-1H-quinolin-2-one (7i). The reaction was carried out according to the previously described procedure for 7-methoxy-3,4-dihydro-1H-quinolin-2-one (7e) using 4-(4'-methoxyphenyl)-2-nitroaniline $(5 \mathrm{~m})(0.040 \mathrm{~g}, 0.16 \mathrm{mmol})$, polymer-supported nitrite $\left(0.14 \mathrm{~g}\right.$, containing $0.48 \mathrm{mmol}$ of $\left.\mathrm{NO}_{2}\right), p$ toluenesulfonic acid monohydrate $(0.083 \mathrm{~g}, 0.48 \mathrm{mmol})$, palladium(II) acetate $(0.0020 \mathrm{~g}, 0.0080 \mathrm{mmol})$, and methyl acrylate $(0.072 \mathrm{~mL}$, $0.80 \mathrm{mmol})$. Purification by silica gel flash column chromatography, eluting with $60 \%$ ethyl acetate in hexane gave $7-\left(4^{\prime}\right.$-methoxyphenyl)3,4-dihydro- $1 \mathrm{H}$-quinolin-2-one $(7 \mathbf{i})(0.023 \mathrm{~g}, 57 \%)$ as a white solid. Mp 145-150 ${ }^{\circ} \mathrm{C}$; IR (neat) 2963, 1671, 1589, 1183, 1023, $807 \mathrm{~cm}^{-1}$; ${ }^{1} \mathrm{H}$ NMR $\left(400 \mathrm{MHz}, \mathrm{CDCl}_{3}\right) \delta 2.67(\mathrm{t}, J=7.2 \mathrm{~Hz}, 2 \mathrm{H}), 2.99(\mathrm{t}, J=$ $7.2 \mathrm{~Hz}, 2 \mathrm{H}), 3.84(\mathrm{~s}, 3 \mathrm{H}), 6.93-6.99(\mathrm{~m}, 3 \mathrm{H}), 7.16(\mathrm{dd}, J=7.8,1.6$ $\mathrm{Hz}, 1 \mathrm{H}), 7.19(\mathrm{~d}, J=7.8 \mathrm{~Hz}, 1 \mathrm{H}), 7.48(\mathrm{~d}, J=8.7 \mathrm{~Hz}, 2 \mathrm{H}), 8.83(\mathrm{br} \mathrm{s}$, $1 \mathrm{H}) ;{ }^{13} \mathrm{C}$ NMR $\left(101 \mathrm{MHz}, \mathrm{CDCl}_{3}\right) \delta 25.1\left(\mathrm{CH}_{2}\right), 30.9\left(\mathrm{CH}_{2}\right), 55.4$ $\left(\mathrm{CH}_{3}\right), 113.7(\mathrm{CH}), 114.3(2 \times \mathrm{CH}), 121.5(\mathrm{CH}), 122.1(\mathrm{C}), 128.1$ $(2 \times \mathrm{CH}), 128.3(\mathrm{CH}), 132.9(\mathrm{C}), 137.7(\mathrm{C}), 140.6(\mathrm{C}), 159.3(\mathrm{C})$, 172.0 (C); MS (ESI) $m / z 276\left(\mathrm{M}+\mathrm{Na}^{+}, 100\right)$; HRMS (ESI) calcd for $\mathrm{C}_{16} \mathrm{H}_{15} \mathrm{NNaO}_{2}\left(\mathrm{M}+\mathrm{Na}^{+}\right) 276.0995$, found 276.0982 .

7-(4'-Fluorophenyl)-3,4-dihydro-1H-quinolin-2-one (7j). The reaction was carried out according to the previously described procedure for 7-methoxy-3,4-dihydro- $1 \mathrm{H}$-quinolin-2-one (7e) using 4-(4'-fluorophenyl)-2-nitroaniline (5n) $(0.050 \mathrm{~g}, 0.17 \mathrm{mmol})$, polymer-supported nitrite $\left(0.15 \mathrm{~g}\right.$, containing $0.51 \mathrm{mmol}$ of $\left.\mathrm{NO}_{2}\right)$, $p$-toluenesulfonic acid monohydrate $(0.088 \mathrm{~g}, 0.51 \mathrm{mmol})$, palladium(II) acetate $(0.0020 \mathrm{~g}, 0.0090 \mathrm{mmol})$, and methyl acrylate $(0.076 \mathrm{~mL}$, $0.85 \mathrm{mmol}$ ). Purification by silica gel flash column chromatography, eluting with $40 \%$ ethyl acetate in hexane gave 7 -(4'-fluorophenyl)-3,4dihydro- $1 H$-quinolin-2-one $(7 \mathbf{j})(0.030 \mathrm{~g}, 73 \%)$ as a white solid. $\mathrm{Mp}$ 141-143 ${ }^{\circ} \mathrm{C}$; IR (neat) 2924, 1674, 1487, 1395, 1219, $812 \mathrm{~cm}^{-1}$; ${ }^{1} \mathrm{H}$ $\operatorname{NMR}\left(400 \mathrm{MHz}, \mathrm{CDCl}_{3}\right) \delta 2.68(\mathrm{dd}, J=9.2,8.0 \mathrm{~Hz}, 2 \mathrm{H}), 3.01$ (dd, $J$ $=8.0,7.2 \mathrm{~Hz}, 2 \mathrm{H}), 6.96(\mathrm{~d}, J=1.8 \mathrm{~Hz}, 1 \mathrm{H}), 7.08-7.14(\mathrm{~m}, 2 \mathrm{H}), 7.16$ (dd, $J=7.8,1.8 \mathrm{~Hz}, 1 \mathrm{H}), 7.22(\mathrm{~d}, J=7.8 \mathrm{~Hz}, 1 \mathrm{H}), 7.47-7.54(\mathrm{~m}$, $2 \mathrm{H}), 8.76(\mathrm{br} \mathrm{s}, 1 \mathrm{H}) ;{ }^{13} \mathrm{C} \mathrm{NMR}\left(101 \mathrm{MHz}, \mathrm{CDCl}_{3}\right) \delta 25.1\left(\mathrm{CH}_{2}\right)$, $30.8\left(\mathrm{CH}_{2}\right), 113.9(\mathrm{CH}), 115.7\left(\mathrm{~d},{ }^{2} J_{\mathrm{C}-\mathrm{F}}=21.5 \mathrm{~Hz}, 2 \times \mathrm{CH}\right), 121.8$ $(\mathrm{CH}), 122.7(\mathrm{C}), 128.4(\mathrm{CH}), 128.6\left(\mathrm{~d},{ }^{3} J_{\mathrm{C}-\mathrm{F}}=8.1 \mathrm{~Hz}, 2 \times \mathrm{CH}\right)$, $136.5\left(\mathrm{~d},{ }^{4} J_{\mathrm{C}-\mathrm{F}}=3.2 \mathrm{~Hz}, \mathrm{C}\right), 137.8(\mathrm{C}), 140.0(\mathrm{C}), 162.6\left(\mathrm{~d},{ }^{1} J_{\mathrm{C}-\mathrm{F}}=\right.$ $247.8 \mathrm{~Hz}, \mathrm{C}$ ), 171.9 (C); MS (EI) m/z $241\left(\mathrm{M}^{+}, 100\right), 213$ (38), 186
(16), 170 (8), 133 (5), 83 (12); HRMS (EI) calcd for $\mathrm{C}_{15} \mathrm{H}_{12} \mathrm{FNO}$ $\left(\mathrm{M}^{+}\right), 241.0903$, found 241.0909 .

tert-Butyl 2-(7'-Methoxy-3', 4'-dihydro-1H-quinolin-2'-one-1'yl)acetate (8). To a stirred solution of 7-methoxy-3,4-dihydro- $1 \mathrm{H}$ quinolin-2-one $(7 \mathrm{e})(0.050 \mathrm{~g}, 0.23 \mathrm{mmol})$ in dry $\mathrm{N}, \mathrm{N}$-dimethylformamide $(7 \mathrm{~mL})$ was added a $60 \%$ dispersion of sodium hydride in mineral oil $(0.043 \mathrm{mg}, 1.1 \mathrm{mmol})$, and the resulting mixture was stirred for $0.25 \mathrm{~h}$ at room temperature. tert-Butyl chloroacetate $(0.20$ $\mathrm{mL}, 1.4 \mathrm{mmol}$ ) was added dropwise and the reaction mixture was then stirred for $1.5 \mathrm{~h}$. Methanol $(6 \mathrm{~mL})$ was added and the mixture was concentrated in vacuo. The reaction mixture was diluted with ethyl acetate $(20 \mathrm{~mL})$, washed with a $5 \%$ aqueous solution of lithium chloride $(3 \times 20 \mathrm{~mL})$ and brine $(20 \mathrm{~mL})$. The organic phase was dried $\left(\mathrm{MgSO}_{4}\right)$, filtered and concentrated. Purification by silica gel flash chromatography, eluting with $20 \%$ ethyl acetate in hexane gave tert-butyl 2-( $7^{\prime}$-methoxy-3', $4^{\prime}$-dihydro- $1 H$-quinolin-2' -one- $1^{\prime}$-yl)acetate $(8)(0.067 \mathrm{~g}, 100 \%)$ as a yellow solid. Mp 57-59 ${ }^{\circ} \mathrm{C}$; IR (neat) $2978,1743,1677,1616,1367,1168,1153,747 \mathrm{~cm}^{-1} ;{ }^{1} \mathrm{H}$ NMR $\left(400 \mathrm{MHz}, \mathrm{CDCl}_{3}\right) \delta 1.44(\mathrm{~s}, 9 \mathrm{H}), 2.66(\mathrm{dd}, J=9.2,8.0 \mathrm{~Hz}$, $2 \mathrm{H}), 2.85$ (dd, $J=9.2,8.0 \mathrm{~Hz}, 2 \mathrm{H}), 3.76(\mathrm{~s}, 3 \mathrm{H}), 4.53(\mathrm{~s}, 2 \mathrm{H}), 6.31$ $(\mathrm{d}, J=2.4 \mathrm{~Hz}, 1 \mathrm{H}), 6.52(\mathrm{dd}, J=8.4,2.4 \mathrm{~Hz}, 1 \mathrm{H}), 7.05(\mathrm{~d}, J=8.4 \mathrm{~Hz}$, $1 \mathrm{H}) ;{ }^{13} \mathrm{C}$ NMR $\left(101 \mathrm{MHz}, \mathrm{CDCl}_{3}\right) \delta 24.6\left(\mathrm{CH}_{2}\right), 28.0\left(3 \times \mathrm{CH}_{3}\right)$, $31.8\left(\mathrm{CH}_{2}\right), 45.0\left(\mathrm{CH}_{2}\right), 55.4\left(\mathrm{CH}_{3}\right), 82.1(\mathrm{C}), 102.0(\mathrm{CH}), 107.0$ $(\mathrm{CH}), 118.4(\mathrm{C}), 128.5(\mathrm{CH}), 140.7$ (C), $159.2(\mathrm{C}), 167.7$ (C), 170.6 (C); MS (EI) m/z $291\left(\mathrm{M}^{+}, 99\right), 235$ (80), 218 (35), 190 (98), 162 (100), 148 (47), 121 (18), 57 (58); HRMS (EI) calcd for $\mathrm{C}_{16} \mathrm{H}_{21} \mathrm{NO}_{4}\left(\mathrm{M}^{+}\right)$291.1471, found 291.1484.

tert-Butyl 2-(6'-Bromo-7'-methoxy-3', 4'-dihydro- $1 \mathrm{H}$-quinolin2'-one-1'-yl)acetate (9). Iron(III) chloride $(0.60 \mathrm{mg}, 0.0038$ mmol) and 1-butyl-3-methylimidazolium bis (trifluoromethanesulfonyl)imide ([BMIM $\left.] \mathrm{NTf}_{2}\right) \quad(3.3 \mu \mathrm{L}, 0.011$ $\mathrm{mmol}$ ) were stirred for $0.5 \mathrm{~h}$ at room temperature. This was added to a solution of tert-butyl 2-( $7^{\prime}$-methoxy- $3^{\prime}, 4^{\prime}$-dihydro- $1 H$-quinolin$2^{\prime}$-one- $1^{\prime}$-yl $)$ acetate $(8)(0.045 \mathrm{~g}, 0.15 \mathrm{mmol})$ and $N$-bromosuccinimide $(0.027 \mathrm{~g}, 0.15 \mathrm{mmol})$ in toluene $(0.5 \mathrm{~mL})$. The reaction mixture was stirred at $40{ }^{\circ} \mathrm{C}$ for $24 \mathrm{~h}$, cooled to room temperature, and concentrated in vacuo. Purification by silica gel flash chromatography, eluting with $30 \%$ ethyl acetate in hexane gave tert-butyl 2-(6'-bromo$7^{\prime}$-methoxy-3',4'-dihydro- $1 H$-quinolin-2'-one- $1^{\prime}$-yl)acetate (9) (0.045 $\mathrm{g}, 81 \%)$ as a pale yellow solid. Mp $70-72^{\circ} \mathrm{C}$; IR (neat) 2977,1740 , $1678,1605,1413,1354,1152,748 \mathrm{~cm}^{-1}$; ${ }^{1} \mathrm{H}$ NMR $(400 \mathrm{MHz}$, $\left.\mathrm{CDCl}_{3}\right) \delta 1.44(\mathrm{~s}, 9 \mathrm{H}), 2.68(\mathrm{dd}, J=9.4,8.0 \mathrm{~Hz}, 2 \mathrm{H}), 2.86(\mathrm{dd}, J=$ 9.4, 8.0 Hz, 2H), 3.85 (s, 3H), $4.58(\mathrm{~s}, 2 \mathrm{H}), 6.30(\mathrm{~s}, 1 \mathrm{H}), 7.33$ (s, $1 \mathrm{H}) ;{ }^{13} \mathrm{C}$ NMR $\left(101 \mathrm{MHz}, \mathrm{CDCl}_{3}\right) \delta 24.3\left(\mathrm{CH}_{2}\right), 28.0\left(3 \times \mathrm{CH}_{3}\right)$, $31.5\left(\mathrm{CH}_{2}\right), 45.1\left(\mathrm{CH}_{2}\right), 56.4\left(\mathrm{CH}_{3}\right), 82.4(\mathrm{C}), 99.8(\mathrm{CH}), 104.8$ (C), $119.7(\mathrm{C}), 132.1(\mathrm{CH}), 140.0(\mathrm{C}), 155.2(\mathrm{C}), 167.6(\mathrm{C}), 170.2$ (C); MS (EI) m/z 369 (M+, 81), 315 (100), 313 (99), 270 (52), 268 (52), 242 (39), 240 (41), 160 (38), 147 (26), 57 (74); HRMS (EI) calcd for $\mathrm{C}_{16} \mathrm{H}_{20}{ }^{79} \mathrm{BrNO}_{4}\left(\mathrm{M}^{+}\right)$369.0576, found 369.0569 .

tert-Butyl 2-[6'-(4' '-Chlorophenyl)-7'-methoxy-3', 4'-dihydro- $1 \mathrm{H}$ quinolin-2'-one-1'-yl]acetate (10). To a stirred solution of tert-butyl 2 -(6'-bromo- $7^{\prime}$-methoxy- $3^{\prime}, 4^{\prime}$-dihydro- $1 H$-quinolin-2' -one- $1^{\prime}$-yl $)$ acetate $(9)(0.080 \mathrm{~g}, 0.22 \mathrm{mmol})$ in $\mathrm{N}, \mathrm{N}$-dimethylformamide $(5 \mathrm{~mL})$ was added 4-chlorophenyl boronic acid (0.041 g, $0.26 \mathrm{mmol})$, a solution of sodium hydrogen carbonate $(0.074 \mathrm{~g}, 0.88 \mathrm{mmol})$ in water $(1 \mathrm{~mL})$, and tetrakis(triphenylphospine)palladium $(0)(0.013 \mathrm{~g}, 0.011$ $\mathrm{mmol}$ ). The mixture was degassed with argon under sonication for 0.5 $\mathrm{h}$ and then heated to $120{ }^{\circ} \mathrm{C}$ for $24 \mathrm{~h}$. The reaction mixture was cooled to room temperature and filtered through a short pad of Celite, which was washed with ethyl acetate $(20 \mathrm{~mL})$. The filtrate was washed with a $5 \%$ aqueous solution of lithium chloride $(3 \times 20 \mathrm{~mL})$ and brine $(20 \mathrm{~mL})$. The organic phase was dried $\left(\mathrm{MgSO}_{4}\right)$, filtered, and concentrated in vacuo. Purification by silica gel flash chromatography, eluting with $30 \%$ ethyl acetate in hexane gave tertbutyl 2-[6'-(4' -chlorophenyl)-7'-methoxy-3', $4^{\prime}$-dihydro- $1 H$-quinolin$2^{\prime}$-one- $1^{\prime}$-yl $]$ acetate $(\mathbf{1 0})(0.066 \mathrm{~g}, 75 \%)$ as a white solid. Mp 114$116{ }^{\circ} \mathrm{C}$; IR (neat) 2980, 1734, 1678, 1614, 1489, 1352, 1155, 833, $748 \mathrm{~cm}^{-1}$; ${ }^{1} \mathrm{H}$ NMR $\left(400 \mathrm{MHz}, \mathrm{CDCl}_{3}\right) \delta 1.47(\mathrm{~s}, 9 \mathrm{H}), 2.73(\mathrm{dd}, J=$ 9.2, $7.6 \mathrm{~Hz}, 2 \mathrm{H}), 2.91(\mathrm{dd}, J=9.2,7.6 \mathrm{~Hz}, 2 \mathrm{H}), 3.76(\mathrm{~s}, 3 \mathrm{H}), 4.63(\mathrm{~s}$, $2 \mathrm{H}), 6.37(\mathrm{~s}, 1 \mathrm{H}), 7.09(\mathrm{~s}, 1 \mathrm{H}), 7.35(\mathrm{~d}, J=8.8 \mathrm{~Hz}, 2 \mathrm{H}), 7.43(\mathrm{~d}, J=$ 
$8.8 \mathrm{~Hz}, 2 \mathrm{H}) ;{ }^{13} \mathrm{C}$ NMR $\left(101 \mathrm{MHz}, \mathrm{CDCl}_{3}\right) \delta 24.5\left(\mathrm{CH}_{2}\right), 28.0(3 \times$ $\left.\mathrm{CH}_{3}\right), 31.8\left(\mathrm{CH}_{2}\right), 45.0\left(\mathrm{CH}_{2}\right), 55.8\left(\mathrm{CH}_{3}\right), 82.3(\mathrm{C}), 99.0(\mathrm{CH})$, $118.4(\mathrm{C}), 123.9(\mathrm{C}), 128.3(2 \times \mathrm{CH}), 129.9(\mathrm{CH}), 130.7(2 \times \mathrm{CH})$, 132.8 (C), 136.1 (C), 140.1 (C), 155.7 (C), 167.8 (C), 170.4 (C); MS (EI) $m / z 401\left(\mathrm{M}^{+}, 57\right), 345$ (100), 272 (34), 258 (11), 84 (12), 57 (18); HRMS (EI) calcd for $\mathrm{C}_{22} \mathrm{H}_{24}{ }^{35} \mathrm{ClNO}_{4}\left(\mathrm{M}^{+}\right) 401.1394$, found 401.1378 .

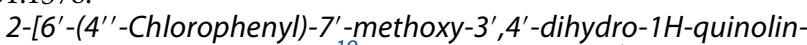
$2^{\prime}$-one- $1^{\prime}$-yl]acetic Acid (11). ${ }^{19}$ Trifluoroacetic acid $(0.050 \mathrm{~mL}, 0.65$ $\mathrm{mmol})$ was added dropwise to a solution of tert-butyl 2-[6'-(4' chlorophenyl)-7'-methoxy-3', $4^{\prime}$-dihydro- $1 H$-quinolin-2 ${ }^{\prime}$-one- $1^{\prime}$-yl $]$ acetate $(10)(0.065 \mathrm{~g}, 0.16 \mathrm{mmol})$ in dry dichloromethane $(3 \mathrm{~mL})$ and stirred under reflux for 5 days. The reaction mixture was then concentrated in vacuo. Purification by silica gel flash chromatography, eluting with $1 \%$ acetic acid and $3 \%$ methanol in dichloromethane gave 2 - [6'-(4' -chlorophenyl)-7'-methoxy-3', $4^{\prime}$-dihydro- $1 H$-quinolin- $2^{\prime}$ one-1'-yl]acetic acid (11) (0.052 g, 92\%) as a white solid. Spectroscopic data were consistent with the literature. ${ }^{19} \mathrm{Mp} 218-$ $220{ }^{\circ} \mathrm{C}$; TFA was added to NMR samples to aid dissolution in $\mathrm{CDCl}_{3} .{ }^{1} \mathrm{H}$ NMR $\left(400 \mathrm{MHz}, \mathrm{CDCl}_{3}+10 \% \mathrm{TFA}\right) \delta 2.82-2.90(\mathrm{~m}$, $2 \mathrm{H}), 2.93-3.02(\mathrm{~m}, 2 \mathrm{H}), 3.80(\mathrm{~s}, 3 \mathrm{H}), 4.85(\mathrm{~s}, 2 \mathrm{H}), 6.49(\mathrm{~s}, 1 \mathrm{H})$, $7.18(\mathrm{~s}, 1 \mathrm{H}), 7.38(\mathrm{~d}, J=8.4 \mathrm{~Hz}, 2 \mathrm{H}), 7.43(\mathrm{~d}, J=8.4 \mathrm{~Hz}, 2 \mathrm{H}) ;{ }^{13} \mathrm{C}$ NMR $\left(101 \mathrm{MHz}, \mathrm{CDCl}_{3}+10 \%\right.$ TFA $) \delta 23.9\left(\mathrm{CH}_{2}\right), 31.2\left(\mathrm{CH}_{2}\right)$, $45.2\left(\mathrm{CH}_{2}\right), 56.6\left(\mathrm{CH}_{3}\right), 100.1(\mathrm{CH}), 118.9(\mathrm{C}), 126.5(\mathrm{C}), 128.6(2$ $\times \mathrm{CH}), 130.6(\mathrm{CH}), 130.8(2 \times \mathrm{CH}), 133.6(\mathrm{C}), 135.7(\mathrm{C}), 138.4$ (C), 156.3 (C), 174.7 (C), 174.8 (C); MS (EI) m/z $345\left(\mathrm{M}^{+}, 100\right)$, 272 (33), 248 (18), 165 (10), 109 (17), 97 (22), 69 (23), 55 (49).

\section{ASSOCIATED CONTENT}

\section{S Supporting Information}

The Supporting Information is available free of charge on the ACS Publications website at DOI: 10.1021/acs.joc.8b01910.

$$
{ }^{1} \mathrm{H} \text { and }{ }^{13} \mathrm{C} \text { NMR spectra for all compounds (PDF) }
$$

\section{AUTHOR INFORMATION}

\section{Corresponding Author}

*E-mail: Andrew.Sutherland@glasgow.ac.uk.

\section{ORCID $\odot$}

Andrew Sutherland: 0000-0001-7907-5766

\section{Notes}

The authors declare no competing financial interest.

\section{ACKNOWLEDGMENTS}

Financial support from the University of Glasgow (studentship to R.J.F.) and EPSRC (EP/K503903/1) is gratefully acknowledged.

\section{REFERENCES}

(1) (a) Mizoroki, T.; Mori, K.; Ozaki, A. Arylation of Olefin with Aryl Iodide Catalyzed by Palladium. Bull. Chem. Soc. Jpn. 1971, 44, 581. (b) Heck, R. F.; Nolley, J. P., Jr. Palladium-Catalyzed Vinylic Hydrogen Substitution Reactions with Aryl, Benzyl and Styryl Halides. J. Org. Chem. 1972, 37, 2320-2322.

(2) For reviews, see: (a) Beletskaya, I. P.; Cheprakov, A. V. The Heck Reaction as a Sharpening Stone of Palladium Catalysis. Chem. Rev. 2000, 100, 3009-3066. (b) Whitcombe, N. J.; Hii, K. K.; Gibson, S. E. Advances in the Heck Chemistry of Aryl Bromides and Chlorides. Tetrahedron 2001, 57, 7449-7476. (c) Handbook of Organopalladium Chemistry for Organic Synthesis; Negishi, E. I., Ed.; Wiley: New York, 2002; Vol. 2. (d) Phan, N. T. S.; van der Sluys, M.; Jones, C. W. On the Nature of the Active Species in Palladium Catalyzed Mizoroki-Heck and Suzuki-Miyaura Couplings Homogeneous or Heterogeneous Catalysis, A Critical Review. Adv. Synth. Catal. 2006, 348, 609-679. (e) Polshettiwar, V.; Molnár, Á. Silica-Supported Pd Catalysts for Heck Coupling Reactions. Tetrahedron 2007, 63, 6949-6976.
(3) (a) Kikukawa, K.; Matsuda, T. Reaction of Diazonium Salts with Transition Metals. I. Arylation of Olefins with Arenediazonium Salts Catalyzed by Zero Valent Palladium. Chem. Lett. 1977, 6, 159-162. (b) Kikukawa, K.; Nagira, K.; Matsuda, T. Synthesis of 4'-Vinylbenzo15-crown-5 from Benzo-15-crown-5. Bull. Chem. Soc. Jpn. 1977, 50, 2207. (c) Kikukawa, K.; Nagira, K.; Terao, N.; Wada, F.; Matsuda, T. Reaction of Diazonium Salts with Transition Metals. II. PalladiumCatalyzed Arylation of Ethylene with Arenediazonium Salts. Bull. Chem. Soc. Jpn. 1979, 52, 2609-2610.

(4) For reviews, see: (a) Roglans, A.; Pla-Quintana, A.; MorenoMañas, M. Diazonium Salts as Substrates in Palladium-Catalyzed Cross-Coupling Reactions. Chem. Rev. 2006, 106, 4622-4643. (b) Taylor, J. G.; Moro, A. V.; Correia, C. R. D. Evolution and Synthetic Applications of the Heck-Matsuda Reaction: The Return of Arenediazonium Salts to Prominence. Eur. J. Org. Chem. 2011, 2011, 1403-1428. (c) Felpin, F.-X.; Nassar-Hardy, L.; Le Callonnec, F.; Fouquet, E. Recent Advances in the Heck-Matsuda Reaction in Heterocyclic Chemistry. Tetrahedron 2011, 67, 2815-2831. (d) Mo, F.; Dong, G.; Zhang, Y.; Wang, J. Recent Applications of Arene Diazonium Salts in Organic Synthesis. Org. Biomol. Chem. 2013, 11, $1582-1593$.

(5) Kikukawa, K.; Nagira, K.; Wada, F.; Matsuda, T. Reaction of Diazonium Salts with Transition Metals-III. Palladium(0)-Catalyzed Arylation of Unsaturated Compounds with Arenediazonium Salts. Tetrahedron 1981, 37, 31-36.

(6) For example, see: (a) Kikukawa, K.; Maemura, K.; Nagira, K.; Wada, F.; Matsuda, T. Direct Transformation of Arylamines to Alkenylarenes Under Palladium(0) Catalysis. Chem. Lett. 1980, 9, 551-552. (b) Akiyama, F.; Miyazaki, H.; Kaneda, K.; Teranishi, S.; Fujiwara, Y.; Abe, M.; Taniguchi, H. Arylation and Alkylation of Olefins by Arylamines or Hydrazines via Carbon-Nitrogen Cleavage in the Presence of Palladium(II) Salts. J. Org. Chem. 1980, 45, 23592361. (c) Kikukawa, K.; Maemura, K.; Kiseki, Y.; Wada, F.; Matsuda, T.; Giam, C. S. Palladium(0)-Catalyzed Arylation of Olefins by Arylamines and an Alkyl Nitrite. J. Org. Chem. 1981, 46, 4885-4888. (d) Sengupta, S.; Bhattacharya, S. Heck Reaction of Arenediazonium Salts: A Palladium-Catalyzed Reaction in an Aqueous Medium. J. Chem. Soc., Perkin Trans. 1 1993, 1943-1947. (e) Coy, E. D.; Jovanovic, L.; Sefkow, M. One-Pot, Pd-Catalyzed Synthesis of transDihydrobenzofurans from o-Aminophenols. Org. Lett. 2010, 12, 1976-1979.

(7) Andrus, M. B.; Song, C.; Zhang, J. Palladium-Imidazolium Carbene Catalyzed Mizoroki-Heck Coupling with Aryl Diazonium Ions. Org. Lett. 2002, 4, 2079-2082.

(8) (a) Felpin, F.-X.; Fouquet, E.; Zakri, C. Heck Cross-Coupling of Aryldiazonium Tetrafluoroborate with Acrylates Catalyzed by Palladium on Charcoal. Adv. Synth. Catal. 2008, 350, 2559-2565. (b) Felpin, F.-X.; Coste, J.; Zakri, C.; Fouquet, E. Preparation of 2Quinolones by Sequential Heck Reduction-Cyclization (HRC) Reactions by Using a Multitask Palladium Catalyst. Chem. - Eur. J. 2009, 15, 7238-7245. (c) Le Callonnec, F.; Fouquet, E.; Felpin, F.-X. Unprecedented Substoichiometric use of Hazardous Aryl Diazonium Salts in the Heck-Matsuda Reaction via a Double Catalytic Cycle. Org. Lett. 2011, 13, 2646-2649. (d) Oger, N.; Le Grognec, E.; Felpin, F.-X. Palladium Nanoparticles Supported on Sulfonic Acid Functionalized Silica as Trifunctional Heterogeneous Catalysts for Heck and Suzuki Reactions. Chem CatChem 2015, 7, 2085-2094.

(9) Filimonov, V. D.; Trusova, M.; Postnikov, P.; Krasnokutskaya, E. A.; Lee, Y. M.; Hwang, H. Y.; Kim, H.; Chi, K.-W. Unusually Stable, Versatile and Pure Arenediazonium Tosylates: Their Preparation, Structures and Synthetic Applicability. Org. Lett. 2008, 10, 39613964.

(10) For example, see: (a) Kutonova, K. V.; Trusova, M. E.; Stankevich, A. V.; Postnikov, P. S.; Filimonov, V. D. Matsuda-Heck Reaction with Arenediazonium Tosylates in Water. Beilstein J. Org. Chem. 2015, 11, 358-362. (b) Kutonova, K. V.; Jung, N.; Trusova, M. E.; Filimonov, V. D.; Postnikov, P. S.; Brase, S. Arenediazonium Tosylates (ADTs) as Efficient Reagents for Suzuki-Miyaura CrossCoupling in Neat Water. Synthesis 2017, 49, 1680-1688. (c) Trusova, 
M. E.; Rodriguez-Zubiri, M.; Kutonova, K. V.; Jung, N.; Bräse, S.; Felpin, F.-X.; Postnikov, P. S. Ultra-Fast Suzuki and Heck Reactions for the Synthesis of Styrenes and Stilbenes using Arenediazonium Salts as Super-Electrophiles. Org. Chem. Front. 2018, 5, 41-45.

(11) (a) Sloan, N. L.; Luthra, S. K.; McRobbie, G.; Pimlott, S. L.; Sutherland, A. A One-Pot Radioiodination of Aryl Amines via Stable Diazonium Salts: Preparation of ${ }^{125}$ I-Imaging Agents. Chem. Commun. 2017, 53, 11008-11011. (b) Sloan, N. L.; Luthra, S. K.; McRobbie, G.; Pimlott, S. L.; Sutherland, A. Late Stage Iodination of Biologically Active Agents using a One-Pot Process from Aryl Amines. RSC Adv. 2017, 7, 54881-54891.

(12) (a) Priego, J.; Carretero, J. C. Asymmetric Heck Reactions of (R)-1-tert-Butylsulfinylcyclopentene with Arenediazonium Salts. Synlett 1999, 1999, 1603-1605. (b) Perez, R.; Veronese, D.; Coelho, F.; Antunes, O. A. C. Palladium Catalyzed Heck Reaction of Arenediazonium Tetrafluoroborate Salts with Baylis-Hillman Adducts: Production of $\alpha$-Benzyl- $\beta$-Keto Esters. Tetrahedron Lett. 2006, 47, 1325-1328.

(13) For examples of chemoselective double cross-coupling reactions of halogenated aryl diazonium salts, see: (a) Sengupta, S.; Sadhukhan, S. K. Iodoarenediazonium Salts: A New Class of Aromatic Substrates for Differential Palladium Catalyzed Reactions. Tetrahedron Lett. 1998, 39, 715-718. (b) Taylor, R. H.; Felpin, F.-X. SuzukiMiyaura Reactions of Arenediazonium Salts Catalyzed by $\mathrm{Pd}(0) / \mathrm{C}$. One-Pot Chemoselective Double Cross-Coupling Reactions. Org. Lett. 2007, 9, 2911-2914. (c) Felpin, F.-X.; Fouquet, E. Efficient and Practical Cross-Coupling of Arenediazonium Tetrafluoroborate Salts with Boronic Acids Catalyzed by Palladium(0)/Barium Carbonate. Adv. Synth. Catal. 2008, 350, 863-868. (d) Peters, M.; Trobe, M.; Tan, H.; Kleineweischede, R.; Breinbauer, R. A Modular Synthesis of Teraryl-Based $\alpha$-Helix Mimetics, Part 1: Synthesis of Core Fragments with Two Electronically Differentiated Leaving Groups. Chem. - Eur. J. 2013, 19, 2442-2449.

(14) One-pot syntheses of compounds 3 and $4 a-4 d$ from 1e using the palladium acetate catalyst for both the initial Heck-Matsuda reaction and the second cross-coupling reaction were investigated. While these did give the desired products, the yields (12-15\%) were significantly lower than synthesizing the compounds via two separate pot processes.

(15) For examples, see: (a) Nakagawa, K.; Murakami, N.; Yoshizaki, S.; Tominaga, M.; Mori, H.; Yabuuchi, Y.; Shintani, S. Derivatives of 3,4-Dihydrocarbostyril as $\beta$-Adrenergic Blocking Agents. J. Med. Chem. 1974, 17, 529-533. (b) Tominaga, M.; Yo, E.; Ogawa, H.; Yamashita, S.; Yabuuchi, Y.; Nakagawa, K. Studies on Positive Inotropic Agents. I. Synthesis of 3,4-Dihydro-6-[4-(3,4-Dimethoxybenzoyl)-1-Piperazinyl]-2(1H)-Quinolinone and Related Compounds. Chem. Pharm. Bull. 1984, 32, 2100-2110. (c) Oshiro, Y.; Sato, S.; Kurahashi, N.; Tanaka, T.; Kikuchi, T.; Tottori, K.; Uwahodo, Y.; Nishi, T. Novel Antipsychotic Agents with Dopamine Autoreceptor Agonist Properties: Synthesis and Pharmacology of 7[4-(4-Phenyl-1-piperazinyl)butoxy]-3,4-dihydro-2 $(1 H)$-quinolinone Derivatives. J. Med. Chem. 1998, 41, 658-667.

(16) Zhou, W.; Zhang, L.; Jiao, N. The Tandem Reaction Combining Radical and Ionic Processes: An Efficient Approach to Substituted 3,4-Dihydroquinolin-2-ones. Tetrahedron 2009, 65, 1982-1987.

(17) Zhang, L.; Sonaglia, L.; Stacey, J.; Lautens, M. Multicomponent Multicatalyst Reactions (MC) ${ }^{2}$ R: One-Pot Synthesis of 3,4Dihydroquinolinones. Org. Lett. 2013, 15, 2128-2131.

(18) In attempting to improve the level of conversion of the electron-rich 2-nitroanilines during the hydrogenation/reduction step of the one-pot process, higher temperatures (e.g., $60{ }^{\circ} \mathrm{C}$ ) at atmospheric pressure were investigated. However, this gave no significant improvement in the conversion. Following this, higher pressures were then investigated. As this was ultimately successful, no alternative reaction conditions or other methods were studied for this step.
(19) Abelman, M.; Chu, N.; Jiang, R.; Leung, K.; Zablocki, J.Fused Heterocyclic Compounds as Ion Channel Modulators. US Patent US20100113514 A1, May 6, 2010.

(20) (a) Racys, D. T.; Warrilow, C. E.; Pimlott, S. L.; Sutherland, A. Highly Regioselective Iodination of Arenes via Iron(III)-Catalyzed Activation of N-Iodosuccinimide. Org. Lett. 2015, 17, 4782-4785. (b) Racys, D. T.; Sharif, S. A. I.; Pimlott, S. L.; Sutherland, A. Silver(I)-Catalyzed Iodination of Arenes: Tuning the Lewis Acidity of N-Iodosuccinimide Activation. J. Org. Chem. 2016, 81, 772-780. (c) Mostafa, M. A. B.; Calder, E. D. D.; Racys, D. T.; Sutherland, A. Intermolecular Aryl C-H Amination Through Sequential Iron and Copper Catalysis. Chem. - Eur. J. 2017, 23, 1044-1047. (d) Mostafa, M. A. B.; Bowley, R. M.; Racys, D. T.; Henry, M. C.; Sutherland, A. Iron(III)-Catalyzed Chlorination of Activated Arenes. J. Org. Chem. 2017, 82, 7529-7537.

(21) Schmidt, B.; Berger, R. A Deacetylation-Diazotation-Coupling Sequence: Palladium-Catalyzed C-C Bond Formation with Acetanilides as Formal Leaving Groups. Adv. Synth. Catal. 2013, 355, 463476.

(22) Pape, S.; Daukšaite, L.; Lucks, S.; Gu, X.; Brunner, H. An In Situ Generated Palladium on Aluminium Oxide: Applications in Gram-Scale Matsuda-Heck Reactions. Org. Lett. 2016, 18, 63766379.

(23) Oger, N.; Le Grognec, E.; Felpin, F.-X. Continuous-Flow Heck-Matsuda Reaction: Homogeneous Versus Heterogeneous Palladium Catalysts. J. Org. Chem. 2014, 79, 8255-8262.

(24) Oger, N.; Le Callonnec, F.; Jacquemin, D.; Fouquet, E.; Le Grognec, E.; Felpin, F.-X. Heck-Matsuda Arylation of Olefins Through a Bicatalytic Approach: Improved Procedures and Rationalization. Adv. Synth. Catal. 2014, 356, 1065-1071.

(25) Dai, M.; Liang, B.; Wang, C.; Chen, J.; Yang, Z. Synthesis of a Novel $\mathrm{C}_{2}$-Symmetric Thiourea and Its Application in the PdCatalyzed Cross-Coupling Reactions with Arenediazonium Salts Under Aerobic Conditions. Org. Lett. 2004, 6, 221-224.

(26) Youn, S. W.; Kim, B. S.; Jagdale, A. R. Pd-Catalyzed Sequential C-C Bond Formation and Cleavage: Evidence for an Unexpected Generation of Arylpalladium(II) Species. J. Am. Chem. Soc. 2012, 134, 11308-11311.

(27) Schmidt, B.; Elizarov, N.; Schilde, U.; Kelling, A. Dual Role of Acetanilides: Traceless Removal of a Directing Group Through Deacetylation/Diazotation and Palladium Catalyzed C-C Coupling Reactions. J. Org. Chem. 2015, 80, 4223-4234.

(28) Chen, G.; Tokunaga, N.; Hayashi, T. Rhodium-Catalyzed Asymmetric 1,4-Addition of Arylboronic Acids to Coumarins: Asymmetric Synthesis of (R)-Tolterodine. Org. Lett. 2005, 7, 22852288.

(29) Zhang, J.-Z.; Tang, Y. Iron-Catalyzed Regioselective Oxo- and Hydroxy-Phthalimidation of Styrenes: Access to $\alpha$-Hydroxyphthalimide Ketones. Adv. Synth. Catal. 2016, 358, 752-764.

(30) Wu, S.; Ma, H.; Jia, X.; Zhong, Y.; Lei, Z. Biopolymer-Metal Complex Wool-Pd as a Highly Active Heterogeneous Catalyst for Heck Reaction in Aqueous Media. Tetrahedron 2011, 67, 250-256.

(31) Burmester, C.; Mataka, S.; Thiemann, T. Synthesis of Nonsymmetric Divinylarenes by a Heck/Wittig Reaction Combination. Synth. Commun. 2010, 40, 3196-3208.

(32) Imashiro, R.; Seki, M. A Catalytic Asymmetric Synthesis of Chiral Glycidic Acid Derivatives Through Chiral Dioxirane-Mediated Catalytic Asymmetric Epoxidation of Cinnamic Acid Derivatives. J. Org. Chem. 2004, 69, 4216-4226.

(33) Hayashi, S.; Ueno, N.; Murase, A.; Nakagawa, Y.; Takada, J. Novel Acid-Type Cyclooxygenase-2 Inhibitors: Design, Synthesis and Structure-Activity Relationship for Anti-Inflammatory Drug. Eur. J. Med. Chem. 2012, 50, 179-195.

(34) Cismesia, M. A.; Ischay, M. A.; Yoon, T. P. Reductive Cyclizations of Nitroarenes to Hydroxamic Acids by Visible Light Photoredox Catalysis. Synthesis 2013, 45, 2699-2705.

(35) Pellicani, R. Z.; Stefanachi, A.; Niso, M.; Carotti, A.; Leonetti, F.; Nicolotti, O.; Perrone, R.; Berardi, F.; Cellamare, S.; Colabufo, N. A. Potent Galloyl-Based Selective Modulators Targeting Multidrug 
Resistance Associated Protein 1 and P-glycoprotein. J. Med. Chem. 2012, 55, 424-436.

(36) Kim, J. S.; Yu, C.; Liu, A.; Liu, L. F.; LaVoie, E. J. Terbenzimidazoles: Influence of 2"-, 4- and 5-Substituents on Cytotoxicity and Relative Potency as Topoisomerase I Poisons. J. Med. Chem. 1997, 40, 2818-2824.

(37) Majellaro, M.; Stefanachi, A.; Tardia, P.; Vicenti, C.; Boccarelli, A.; Pannunzio, A.; Campanella, F.; Coluccia, M.; Denora, N.; Leonetti, F.; de Candia, M.; Altomare, C. D.; Cellamare, S. Investigating Structural Requirements for the Antiproliferative Activity of Biphenyl Nicotinamides. ChemMedChem 2017, 12, $1380-1389$.

(38) Kumar, G. S.; Kumar, P.; Kapur, M. Traceless Directing-Group Strategy in the Ru-Catalyzed, Formal [3 + 3] Annulation of Anilines with Allyl Alcohols: A One-Pot, Domino Approach for the Synthesis of Quinolines. Org. Lett. 2017, 19, 2494-2497.

(39) Bai, P.; Li, Y.-Q.; Huang, Z.-Z. A Four-Component Cascade C$\mathrm{H}$ Functionalization/Cyclization/Nucleophilic Substitution Reaction to Construct $\alpha$-Functionalized Tetrahydroquinolines by the Strategy of In Situ Directing Group Formation. Org. Lett. 2017, 19, 13741377. 